UCB-PTH-02/08

LBNL-49643

SPIN-2002/07

ITP-UU-02/08

ITFA-2002-06

hep-th/0203056

\title{
Two Large Examples in Orbifold Theory: Abelian Orbifolds and the Charge Conjugation Orbifold on $\mathfrak{s u}(n)$
}

\author{
M.B.Halpern ${ }^{a \dagger}$ and N.A.Obers ${ }^{b, c, d \ddagger}$ \\ ${ }^{a}$ Department of Physics, University of California, Berkeley, California 94720, USA \\ and Theoretical Physics Group, Lawrence Berkeley National Laboratory \\ University of California, Berkeley, California 94720, USA \\ ${ }^{b}$ Spinoza Institute and Institute for Theoretical Physics \\ Utrecht University, Leuvenlaan 4, 3584 CE Utrecht, The Netherlands \\ ${ }^{c}$ Institute for Theoretical Physics, University of Amsterdam \\ Valckenierstraat 65, 1018 XE Amsterdam, The Netherlands \\ ${ }^{d}$ The Niels Bohr Institute, Blegdamsvej 17, DK-2100 Copenhagen Ø, Denmark
}

\begin{abstract}
Recently the operator algebra and twisted vertex operator equations were given for each sector of all WZW orbifolds, and a set of twisted KZ equations for the WZW permutation orbifolds were worked out as a large example. In this companion paper we report two further large examples of this development. In the first example we solve the twisted vertex operator equations in an abelian limit to obtain the twisted vertex operators and correlators of a large class of abelian orbifolds. In the second example, the twisted vertex operator equations are applied to obtain a set of twisted $\mathrm{KZ}$ equations for the (outer-automorphic) charge conjugation orbifold on $\mathfrak{s} \mathfrak{u}(n \geq 3)$.
\end{abstract}

\footnotetext{
${ }^{\dagger}$ halpern@physics. berkeley.edu

$\ddagger$ obers@phys.uu.nl
} 


\section{Contents}

1 Introduction 2

2 The Abelian Orbifolds $A_{\text {Cartan } g}(H) / H$

2.1 An Abelian limit of the WZW orbifolds . . . . . . . . . . . . . . . . . . . . . . . 3

2.2 Solution of the twisted left-mover vertex operator equation $\ldots \ldots \ldots \ldots$

2.3 Summary of the twisted left-mover vertex operators . . . . . . . . . . . . . . . . . . . . 9

2.4 Properties of the twisted vertex operators . . . . . . . . . . . . . . . . . . . 10

2.5 Correlators of the twisted vertex operators … . . . . . . . . . . . . . . . . . . . 13

2.6 Example: Abelian permutation orbifolds . . . . . . . . . . . . . . . . . . . . . . . . . . . 14

2.7 Subexample: Abelian cyclic permutation orbifolds . . . . . . . . . . . . . . . . . . . . . . . . 19

2.8 Example: The inversion orbifold $A_{\text {Cartan su(2) }}\left(\mathbb{Z}_{2}\right) / \mathbb{Z}_{2}$. . . . . . . . . . . . . . . . . . . . . . . . . . 22

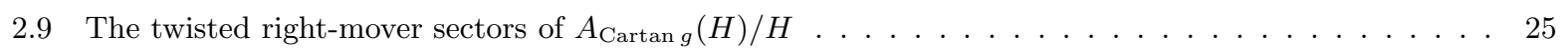

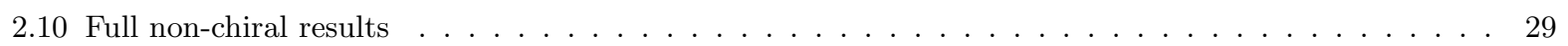

2.11 Example: More about the inversion orbifold . . . . . . . . . . . . . . . . . . . . . . . . 30

3 The Charge Conjugation Orbifold on $\mathfrak{s u}(n)$

3.1 Charge conjugation . . . . . . . . . . . . . . . . . . . . . . . . 32

3.2 The Cartesian form of $A_{n-1}^{(2)} \ldots \ldots \ldots \ldots \ldots$

3.3 The twisted representation matrices . . . . . . . . . . . . . . . . . . . . . . . . . . 37

$3.4 \quad$ Twisted left-mover KZ system for charge conjugation orbifolds . . . . . . . . . . . . . . . . . . . . . 38

3.5 Rectification and the twisted right-mover $\mathrm{KZ} \mathrm{system} \mathrm{\ldots} \mathrm{.} \mathrm{.} \mathrm{.} \mathrm{.} \mathrm{.} \mathrm{.} \mathrm{.} \mathrm{.} \mathrm{.} \mathrm{.} \mathrm{.} \mathrm{.} \mathrm{.} \mathrm{.} \mathrm{.} \mathrm{.} \mathrm{.} \mathrm{.} \mathrm{.} \mathrm{.} \mathrm{.} \mathrm{.} \mathrm{.} \mathrm{41}$

3.6 The classical theory of charge conjugation orbifolds . . . . . . . . . . . . . . . . . . . . . . . 42

3.7 Correlator examples and undetermined parameters . . . . . . . . . . . . . . . . . . . . . . . . . 44

\begin{tabular}{|l|l|}
\hline A The $H$-eigenvalue problem for permutation groups \\
\hline
\end{tabular}

B More about the WZW permutation orbifolds

C An identity for the full correlators of the abelian orbifolds

D Relation to the Dynkin automorphisms

\begin{tabular}{|ll|} 
E & Flat connections in the charge conjugation orbifolds \\
\hline
\end{tabular}

\begin{tabular}{|l|l}
\hline References & 57
\end{tabular}

\section{Introduction}

In the last few years there has been a quiet revolution in the local theory of current-algebraic orbifolds. Building on the discovery of orbifold affine algebra [1, 2] in the cyclic permutation orbifolds, Refs. 3, 44 gave the twisted currents and stress tensor in each sector of any currentalgebraic orbifold $A(H) / H$ - where $A(H)$ is any current-algebraic conformal field theory [5-9] with a finite symmetry group $H$. The construction treats all current-algebraic orbifolds at the same time, using the method of eigenfields and the principle of local isomorphisms to map OPEs in the symmetric theory to OPEs in the orbifold. The orbifold results are expressed in terms of a set of duality transformations, which are discrete Fourier transforms constructed from the eigendata of the $H$-eigenvalue problem. 
More recently, the special case of the WZW orbifolds

$$
\frac{A_{g}(H)}{H}, \quad H \subset \operatorname{Aut}(g)
$$

was worked out in further detail [10], introducing the linkage relation and the extended $H$ eigenvalue problem in order to include the operator algebra and the twisted vertex operator equations of the twisted affine primary fields of the WZW orbifolds. The twisted vertex operator equations set the stage for finding the twisted $K Z$ equations of the WZW orbifolds, and twisted KZ equations for the WZW permutation orbifolds and the inner-automorphic WZW orbifolds were worked out as large examples in Ref. [10].

In this companion paper, we apply the twisted vertex operator equations of Ref. [10] to work out the details of two other large examples. In the first example (see Sec. 2) we solve the twisted vertex operator equations in an abelian limit to obtain the twisted vertex operators and correlators of a large class of abelian orbifolds

$$
\frac{A_{\text {Cartan } g}(H)}{H}, \quad H \subset \operatorname{Aut}(\operatorname{Cartan} g), \quad \text { Cartan } g \subset g
$$

where the ambient algebra $g$ supplies the representation space for the twisted sectors of each orbifold. In the second example (see Sec. 3), we apply the twisted vertex operator equations to obtain a set of twisted $K Z$ equations for the (outer-automorphic) charge conjugation orbifold on $\mathfrak{s u}(n)$

$$
\frac{A_{\mathfrak{s u}(n)}\left(\mathbb{Z}_{2}\right)}{\mathbb{Z}_{2}}, \quad n \geq 3
$$

and some simple solutions of these equations are also discussed.

Subsec. 3.4 also notes a more general twisted KZ system for the correlators in the "scalar" twist-field states of any WZW orbifold. This result includes as special cases the known twisted KZ system for the WZW permutation orbifolds, our twisted KZ system for the charge conjugation orbifold on $\mathfrak{s u}(n)$, and a generalization to every outer-automorphic WZW orbifold.

\section{The Abelian Orbifolds $A_{\text {Cartan } g}(H) / H$}

\subsection{An Abelian limit of the WZW orbifolds}

As our first large example, we consider the class of abelian orbifolds

$$
\frac{A_{\text {Cartan } g}(H)}{H}, \quad H \subset \operatorname{Aut}(\operatorname{Cartan} g)
$$

where $H$ is any finite group and $A_{\text {Cartan } g}(H)$ is any $H$-symmetric conformal field theory constructed from the Cartan subalgebra of a compact semisimple Lie algebra $g$

$$
\text { Cartan } g \subset g, \quad g=\oplus_{I} \mathfrak{g}^{I}, \quad \text { Cartan } g=\oplus_{I} \text { Cartan } \mathfrak{g}^{I} .
$$

In the composite notation of Refs. [2, 3, 4, 10], the left-mover sector of the $H$-symmetric CFT is described by the stress tensor and abelian current algebra

$$
T(z)=\frac{1}{2} G^{a b}: J_{a}(z) J_{b}(z): \quad c=\operatorname{dim}(\operatorname{Cartan} g)
$$




$$
J_{a}(z)=\sum_{m \in \mathbb{Z}} J_{a}(m) z^{-m-1}, \quad\left[J_{a}(m), J_{b}(n)\right]=m G_{a b} \delta_{m+n, 0}, \quad a, b=1 \ldots \operatorname{dim}(\operatorname{Cartan} g)
$$

where : $\cdot$ : is operator product normal ordering and $G_{a b}$ is the induced metric on Cartan $g$. The ambient algebra $g$ provides the induced metric and the representation theory of the symmetric CFT, but is otherwise inactive. The $H$-symmetry of the system is specified as

$$
\begin{gathered}
J_{a}(z)^{\prime}=\omega\left(h_{\sigma}\right)_{a}{ }^{b} J_{b}(z), \quad \omega\left(h_{\sigma}\right)_{a}{ }^{c} \omega\left(h_{\sigma}\right)_{b}{ }^{d} G_{c d}=G_{a b}, \quad \omega\left(h_{\sigma}\right) \in H, \quad \sigma=0, \ldots, N_{c}-1 \\
T(z)^{\prime}=\frac{1}{2} G^{a b}: J_{a}(z)^{\prime} J_{b}(z)^{\prime}:=T(z)
\end{gathered}
$$

where $\omega\left(h_{\sigma}\right)$ is the action of $h_{\sigma} \in H$ and $N_{c}$ is the number of conjugacy classes of $H$.

When needed, the composite notation can be replaced by the explicit notation

$$
\begin{gathered}
a \rightarrow a(I), \quad J_{a} \rightarrow J_{a(I)}, \quad G_{a b} \rightarrow G_{a(I), b(J)}=\oplus_{I} k_{I} \eta_{a(I) b(I)}^{I}, \\
a(I), b(I)=1 \ldots \operatorname{dim}\left(\text { Cartan } \mathfrak{g}^{I}\right)
\end{gathered}
$$

where $\eta_{a(I) b(I)}^{I}$ is the induced Killing metric of Cartan $\mathfrak{g}^{I}$ and $x_{I}=2 k_{I} / \psi_{I}^{2}$ is the invariant level of affine $\mathfrak{g}^{I}$. As an example (see also Subsec. 2.6) permutation-invariant systems satisfy

$$
\begin{gathered}
\mathfrak{g}^{I} \simeq \mathfrak{g}, \quad \operatorname{Cartan} \mathfrak{g}^{I} \simeq \operatorname{Cartan} \mathfrak{g} \\
J_{a(I)}=J_{a I}, \quad k_{I}=k, \quad x_{I}=x, \quad \eta_{a(I) b(I)}^{I}=\eta_{a b}, \quad a=1 \ldots \operatorname{dim}(\operatorname{Cartan} \mathfrak{g})
\end{gathered}
$$

where $\eta_{a b}$ is the induced Killing metric of Cartan $\mathfrak{g}$ and $H$ (permutation) acts among the copies Cartan $\mathfrak{g}^{I}$ of Cartan $\mathfrak{g}$.

The twisted current-algebraic description of $A_{\text {Cartan } g}(H) / H$ can be easily read as the abelian limit $g \rightarrow$ Cartan $g$ of the general WZW orbifold in Refs. [4] or [10]. One finds the stress tensor and twisted current algebra of sector $\sigma$

$$
\begin{gathered}
\hat{T}_{\sigma}(z)=\mathcal{L}_{\hat{\mathfrak{g}}(\sigma)}^{n(r) \mu ;-n(r), \nu}(\sigma): \hat{J}_{n(r) \mu}(z, \sigma) \hat{J}_{-n(r), \nu}(z, \sigma):, \quad \hat{c}(\sigma)=c, \quad \sigma=0, \ldots, N_{c}-1 \\
\hat{J}_{n(r) \mu}\left(z e^{2 \pi i}, \sigma\right)=e^{-2 \pi i \frac{n(r)}{\rho(\sigma)} \hat{J}_{n(r) \mu}(z, \sigma), \quad \hat{J}_{n(r) \pm \rho(\sigma), \mu}(z, \sigma)=\hat{J}_{n(r) \mu}(z, \sigma)} \\
\hat{J}_{n(r) \mu}(z, \sigma)=\sum_{m \in \mathbb{Z}} \hat{J}_{n(r) \mu}\left(m+\frac{n(r)}{\rho(\sigma)}\right) z^{-\left(m+\frac{n(r)}{\rho(\sigma)}\right)-1} \\
{\left[\hat{J}_{n(r) \mu}\left(m+\frac{n(r)}{\rho(\sigma)}\right), \hat{J}_{n(s) \nu}\left(n+\frac{n(s)}{\rho(\sigma)}\right)\right]=\left(m+\frac{n(r)}{\rho(\sigma)}\right) \delta_{m+n+\frac{n(r)+n(s)}{\rho(\sigma)}, 0} \mathcal{G}_{n(r) \mu ;-n(r), \nu}(\sigma)}
\end{gathered}
$$




$$
\hat{J}_{n(r) \pm \rho(\sigma), \mu}\left(m+\frac{n(r) \pm \rho(\sigma)}{\rho(\sigma)}\right)=\hat{J}_{n(r) \mu}\left(m \pm 1+\frac{n(r)}{\rho(\sigma)}\right)
$$

where : $\cdot$ : is operator product normal ordering [2, 3, 11, 4, 10] and the duality transformations [3, 4, 10]

$$
\begin{aligned}
& \mathcal{F}_{n(r) \mu ; n(s) \nu}{ }^{n(t) \delta}(\sigma)=0, \quad \mathcal{L}_{\hat{\mathfrak{g}}(\sigma)}^{n(r) \mu ; n(s) \nu}(\sigma)=\frac{1}{2} \mathcal{G}^{n(r) \mu ; n(s) \nu}(\sigma) \\
& \mathcal{G}_{n(r) \mu ; n(s) \nu}(\sigma)=\chi(\sigma)_{n(r) \mu} \chi(\sigma)_{n(s) \nu} U(\sigma)_{n(r) \mu}{ }^{a} U(\sigma)_{n(s) \nu}{ }^{b} G_{a b} \\
& =\delta_{n(r)+n(s), 0 \bmod \rho(\sigma)} \mathcal{G}_{n(r) \mu ;-n(r), \nu}(\sigma)=\mathcal{G}_{n(r) \pm \rho(\sigma), \mu ; n(s) \nu}(\sigma) \\
& \mathcal{G}^{n(r) \mu ; n(s) \nu}(\sigma)=\chi(\sigma)_{n(r) \mu}^{-1} \chi(\sigma)_{n(s) \nu}^{-1} G^{a b} U^{\dagger}(\sigma)_{a}{ }^{n(r) \mu} U^{\dagger}(\sigma)_{b}{ }^{n(s) \nu} \\
& =\delta_{n(r)+n(s), 0 \bmod \rho(\sigma)} \mathcal{G}^{n(r) \mu ;-n(r), \nu}(\sigma)=\mathcal{G}^{n(r) \pm \rho(\sigma), \mu ; n(s) \nu}(\sigma)
\end{aligned}
$$

are called respectively the twisted structure constants, the twisted inverse inertia tensor, the twisted metric and the twisted inverse metric of sector $\sigma$. The Kronecker forms in (2.8d) and (2.8 ) are the solutions of the selection rules [3, 4, 10] of the twisted tensors. In further detail, the integer $\rho(\sigma)$ is the order of $h_{\sigma} \in H$ while the unitary matrices $U(\sigma)$, the spectral integers $n(r) \equiv n(r, \sigma)$ and the degeneracy indices $\mu \equiv \mu(r, \sigma)$ are determined by solving the $H$-eigenvalue problem [3, 斗, 10]

$$
\omega\left(h_{\sigma}\right) U^{\dagger}(\sigma)=U^{\dagger}(\sigma) E(\sigma), \quad E(\sigma)=e^{-2 \pi i \frac{n(r)}{\rho(\sigma)}}, \quad \sigma=0, \ldots, N_{c}-1 .
$$

The quantities $\chi(\sigma)$ with $\chi(0)=1$ are normalization constants. As seen in Eqs. (2.7-9), all quantities are periodic $n(r) \rightarrow n(r) \pm \rho(\sigma)$ in the spectral integers.

We note in particular that the zero modes $\left\{\hat{J}_{0 \mu}(0)\right\}$ of the twisted current algebra $(2.7 \mathrm{~d})$ commute with themselves and indeed with all the twisted modes

$$
\left[\hat{J}_{0 \mu}(0), \hat{J}_{n(s) \nu}\left(n+\frac{n(s)}{\rho(\sigma)}\right)\right]=0, \quad \forall \mu, \nu, n(s) .
$$

As in the case of the WZW orbifolds [10] the zero modes of the twisted current algebra will contribute to the residual symmetry (global Ward identities) of each orbifold sector $\sigma$ (see Subsecs. 2.5 and 2.6).

The ground state (twist-field state) of sector $\sigma$ satisfies

$$
\hat{J}_{n(r) \mu}\left(m+\frac{n(r)}{\rho(\sigma)} \geq 0\right)|0\rangle_{\sigma}={ }_{\sigma}\langle 0| \hat{J}_{n(r) \mu}\left(m+\frac{n(r)}{\rho(\sigma)} \leq 0\right)=0, \quad \sigma=0, \ldots, N_{c}-1
$$

and so the $M$ or mode-ordered forms of Refs. [4] or [10] are immediately useful. For example the Virasoro generators in sector $\sigma$ of $A_{\text {Cartan } g}(H) / H$

$$
\begin{gathered}
L_{\sigma}(m)=\frac{1}{2} \sum_{r, \mu, \nu} \mathcal{G}^{n(r) \mu ;-n(r), \nu}(\sigma)\left\{\sum_{p \in \mathbb{Z}}: \hat{J}_{n(r) \mu}\left(p+\frac{n(r)}{\rho(\sigma)}\right) \hat{J}_{-n(r), \nu}\left(m-p-\frac{n(r)}{\rho(\sigma)}\right):_{M}\right. \\
\left.+\delta_{m, 0} \frac{\bar{n}(r)}{2 \rho(\sigma)}\left(1-\frac{\bar{n}(r)}{\rho(\sigma)}\right) \mathcal{G}_{n(r) \mu ;-n(r), \nu}(\sigma)\right\} \\
\overline{n(r)} \equiv \bar{n}(r) \equiv n(r)-\rho(\sigma)\left\lfloor\frac{n(r)}{\rho(\sigma)}\right\rfloor, \quad \bar{n}(r) \in\{0, \ldots, \rho(\sigma)-1\}
\end{gathered}
$$


are easily obtained as the abelian limit of the form given for all WZW orbifolds in these references. Here $\bar{n}(r)$ is the pullback of the spectral integer $n(r)$ into the fundamental range $0 \leq \bar{n} \leq \rho(\sigma)-1$. Then the ground state conformal weight of sector $\sigma$

$$
\begin{gathered}
\left(L_{\sigma}(m \geq 0)-\delta_{m, 0} \hat{\Delta}_{0}(\sigma)\right)|0\rangle_{\sigma}=0 \\
\hat{\Delta}_{0}(\sigma)=\frac{1}{2} \sum_{r, s, \mu, \nu} \mathcal{G}^{n(r) \mu ; n(s) \nu}(\sigma) \mathcal{G}_{n(r) \mu ; n(s) \nu}(\sigma) \frac{\bar{n}(r)}{2 \rho(\sigma)}\left(1-\frac{\bar{n}(r)}{\rho(\sigma)}\right) \\
=\frac{1}{2} \sum_{r, \mu(r)} \frac{\bar{n}(r)}{2 \rho(\sigma)}\left(1-\frac{\bar{n}(r)}{\rho(\sigma)}\right), \quad \sigma=0, \ldots, N_{c}-1
\end{gathered}
$$

is easily computed from (2.12) and the duality transformations in (2.8).

Going beyond the general stress tensors of Ref. [4], Ref. [10] also discusses the full chiral algebra of all WZW orbifolds, including the twisted left-mover affine primary fields $\hat{g}_{+}(\mathcal{T}, z, \sigma)$ of sector $\sigma$. For our class of abelian orbifolds the abelian limit $\hat{g}_{+}(\mathcal{T}, z, \sigma)$ is more properly called a twisted left-mover vertex operator. In particular, we read from Ref. [10] that the twisted left-mover vertex operators satisfy

$$
\begin{gathered}
\hat{J}_{n(r) \mu}(z, \sigma) \hat{g}_{+}(\mathcal{T}, w, \sigma)=\frac{\hat{g}_{+}(\mathcal{T}, w, \sigma)}{z-w} \mathcal{T}_{n(r) \mu}(T, \sigma)+\mathcal{O}(z-w)^{0} \\
{\left[\hat{J}_{n(r) \mu}\left(m+\frac{n(r)}{\rho(\sigma)}\right), \hat{g}_{+}(\mathcal{T}, z, \sigma)\right]=\hat{g}_{+}(\mathcal{T}, z, \sigma) \mathcal{T}_{n(r) \mu}(T, \sigma) z^{m+\frac{n(r)}{\rho(\sigma)}}} \\
{\left[L_{\sigma}(m), \hat{g}_{+}(\mathcal{T}, z, \sigma)\right]=z^{m}\left(z \partial_{z}+\mathcal{D}(\mathcal{T}, \sigma)(m+1)\right) \hat{g}_{+}(\mathcal{T}, z, \sigma)} \\
\mathcal{D}(\mathcal{T}, \sigma)=\frac{1}{2} \mathcal{G}^{n(r) \mu ;-n(r), \nu}(\sigma) \mathcal{T}_{n(r) \mu}(T, \sigma) \mathcal{T}_{-n(r), \nu}(T, \sigma) \\
\mathcal{T}_{n(r) \mu}(T, \sigma)_{N(r) \mu}{ }^{N(s) \nu}=\chi(\sigma)_{n(r) \mu} U(\sigma)_{n(r) \mu}{ }^{a}\left(U(T, \sigma) T_{a} U^{\dagger}(T, \sigma)\right)_{N(r) \mu}{ }^{N(s) \nu} \\
=\delta_{\frac{n(r)}{\rho(\sigma)}+\frac{N(r)-N(s)}{R(\sigma)}, 0 \bmod 1} \mathcal{T}_{n(r) \mu}(T, \sigma)_{N(r) \mu}{ }^{N(r)+\frac{R(\sigma)}{\rho(\sigma)} n(r), \nu} \\
{\left[T_{a}, T_{b}\right]=0, \quad a^{a, b=1 \ldots \operatorname{dim}(\operatorname{Cartan} g)}} \\
{\left[\mathcal{T}_{n(r) \mu}(T, \sigma), \mathcal{T}_{n(s) \nu}(T, \sigma)\right]=0, \quad \mathcal{T}_{n(r) \pm \rho(\sigma), \mu}(T, \sigma)=\mathcal{T}_{n(r) \mu}(T, \sigma)}
\end{gathered}
$$

where $\left\{T_{a}\right\}$ are the commuting Cartan matrices of the irreps $\mathbb{T}$ of the ambient Lie algebra $g \supset$ Cartan $g$ and $\left\{\mathcal{T}_{n(r) \mu}(T, \sigma)\right\}$ is another set of duality transformations called the twisted representation matrices of sector $\sigma$. Here $R(\sigma) \equiv R(T, \sigma)$ is the order of the action $W\left(h_{\sigma} ; T\right)$ of $h_{\sigma} \in H$ in

\footnotetext{
${ }^{\ddagger 1}$ Although it is not mentioned explicitly below, one should choose only those irreps of $g$ consistent with the affine cutoff $T \leq T_{x}$ at invariant level $x=\left\{x_{I}\right\}$ of affine $g$.
} 
representation $T$ of $g$, while the unitary matrices $U(T, \sigma)$, the spectral integers $N(r) \equiv N(r, T, \sigma)$ and the degeneracy indices $\mu \equiv \mu(r, T, \sigma)$ are obtained as the eigendata of the extended $H$ eigenvalue problem 10]

$$
W\left(h_{\sigma} ; T\right) U^{\dagger}(T, \sigma)=U^{\dagger}(T, \sigma) E(T, \sigma), \quad E(T, \sigma)=e^{-2 \pi i \frac{N(r)}{R(\sigma)}} .
$$

Again all quantities are periodic $N(r) \rightarrow N(r) \pm R(\sigma)$ in the spectral integers. These results are essentially unchanged from Ref. [10], except that in our case the twisted representation matrices commute because the $T_{a}$ 's commute. In what follows we generally abbreviate $\hat{J}(z) \equiv \hat{J}(z, \sigma)$ and $\mathcal{T} \equiv \mathcal{T}(T, \sigma)$

\subsection{Solution of the twisted left-mover vertex operator equation}

Ref. [10] also gives the operator equations of motion, called the twisted vertex operator equations, for the twisted affine-primary fields in each sector of all WZW orbifolds. In particular, the abelian limit of the twisted left-mover vertex operator equation

$$
\begin{gathered}
\partial \hat{g}_{+}(\mathcal{T}, z, \sigma)=\mathcal{G}^{n(r) \mu ;-n(r), \nu}(\sigma)\left(: \hat{J}_{n(r) \mu}(z) \hat{g}_{+}(\mathcal{T}, z, \sigma):_{M}-\frac{\bar{n}(r)}{\rho(\sigma)} \frac{1}{z} \hat{g}_{+}(\mathcal{T}, z, \sigma) \mathcal{T}_{n(r) \mu}\right) \mathcal{T}_{-n(r), \nu} \\
: \hat{J}_{n(r) \mu}(z) \hat{g}_{+}(\mathcal{T}, z, \sigma):_{M}=\hat{J}_{n(r) \mu}^{-}(z) \hat{g}_{+}(\mathcal{T}, z, \sigma)+\hat{g}_{+}(\mathcal{T}, z, \sigma) \hat{J}_{n(r) \mu}^{+}(z) \\
\hat{J}_{n(r) \mu}^{-}(z) \equiv \sum_{m \leq-1} \hat{J}_{\bar{n}(r) \mu}\left(m+\frac{\bar{n}(r)}{\rho(\sigma)}\right) z^{-\left(m+\frac{\bar{n}(r)}{\rho(\sigma)}\right)-1}, \hat{J}_{n(r) \mu}^{+}(z) \equiv \sum_{m \geq 0} \hat{J}_{\bar{n}(r) \mu}\left(m+\frac{\bar{n}(r)}{\rho(\sigma)}\right) z^{-\left(m+\frac{\bar{n}(r)}{\rho(\sigma)}\right)-1} \\
{\left[\hat{J}_{n(r) \mu}^{+}(z), \hat{J}_{n(s) \nu}^{+}(w)\right]=\left[\hat{J}_{n(r) \mu}^{-}(z), \hat{J}_{n(s) \nu}^{-}(w)\right]=0}
\end{gathered}
$$

is also easily read from Ref. [10]. Here the form is unchanged from Ref. 10] except for the vanishing commutators (2.16d) of the partial currents $\hat{J}^{ \pm}$, which reflect the abelian character of the present case.

The central task of this section is to solve the twisted vertex operator equation (2.16a), following the general strategy given for the untwisted WZW vertex operator equation and an abelian limit of this equation in Ref. [12]. In what follows, we will assume that the twisted vertex operator $\hat{g}_{+}$is a square matrix and that the twisted representation matrices $\mathcal{T}$ commute with everything, including the solution:

$$
\hat{g}_{+}(\mathcal{T}, z, \sigma)_{N(r) \mu}{ }^{N(s) \nu}, \quad\left[\mathcal{T}_{n(r) \mu}, \hat{g}_{+}(\mathcal{T}, z, \sigma)\right]=0
$$

This assumption will be justified a posteriori by our solution below.

The first step in the solution is the direct integration

$$
\begin{aligned}
\hat{g}_{+}(\mathcal{T}, z, \sigma)= & \left(\frac{z}{z_{0}}\right)^{-\mathcal{G}^{n(r) \mu ;-n(r), \nu}(\sigma) \frac{\bar{n}(r)}{\rho(\sigma)} \mathcal{T}_{n(r) \mu} \mathcal{T}_{-n(r), \nu}} \\
& \times \exp \left(\int_{z_{0}}^{z} d z^{\prime} \mathcal{G}^{n(r) \mu ;-n(r), \nu}(\sigma) \hat{J}_{n(r) \mu}^{-}\left(z^{\prime}\right) \mathcal{T}_{-n(r), \nu}\right) \\
& \times \hat{g}_{+}\left(\mathcal{T}, z_{0}, \sigma\right) \exp \left(\int_{z_{0}}^{z} d z^{\prime} \mathcal{G}^{n(r) \mu ;-n(r), \nu}(\sigma) \hat{J}_{n(r) \mu}^{+}\left(z^{\prime}\right) \mathcal{T}_{-n(r), \nu}\right)
\end{aligned}
$$


which follows using (2.16d) and (2.17). The reference point $z_{0}$ in (2.18) is arbitrary.

The integrals in (2.18) are easily performed and the results rearranged in the form

$$
\begin{aligned}
& \hat{g}_{+}(\mathcal{T}, z, \sigma)=z^{-\mathcal{G}^{n(r) \mu ;-n(r), \nu}(\sigma) \frac{\bar{n}(r)}{\rho(\sigma)} \mathcal{T}_{n(r) \mu} \mathcal{T}_{-n(r), \nu}} \hat{V}_{-}(\mathcal{T}, z, \sigma) \hat{G}_{+}\left(\mathcal{T}, z_{0}, \sigma\right) \\
& \times z^{\hat{J}_{0 \mu}(0) \mathcal{G}^{0 \mu ; 0 \nu}(\sigma) \mathcal{T}_{0 \nu}} \hat{V}_{+}^{(0)}(\mathcal{T}, z, \sigma) \hat{V}_{+}(\mathcal{T}, z, \sigma) \\
& \hat{V}_{-}(\mathcal{T}, z, \sigma) \equiv \exp \left\{-\mathcal{G}^{n(r) \mu ;-n(r), \nu}(\sigma) \sum_{m \leq-1} \hat{J}_{\bar{n}(r) \mu}\left(m+\frac{\bar{n}(r)}{\rho(\sigma)}\right) \frac{z^{-\left(m+\frac{\bar{n}(r)}{\rho(\sigma)}\right)}}{m+\frac{\bar{n}(r)}{\rho(\sigma)}} \mathcal{T}_{-n(r), \nu}\right\} \\
& \hat{V}_{+}(\mathcal{T}, z, \sigma) \equiv \exp \left\{-\mathcal{G}^{n(r) \mu ;-n(r), \nu}(\sigma)\left(1-\delta_{\bar{n}(r), 0}\right) \sum_{m \geq 0} \hat{J}_{\bar{n}(r) \mu}\left(m+\frac{\bar{n}(r)}{\rho(\sigma)}\right) \frac{z^{-\left(m+\frac{\bar{n}(r)}{\rho(\sigma)}\right)}}{m+\frac{\bar{n}(r)}{\rho(\sigma)}} \mathcal{T}_{-n(r), \nu}\right\} \\
& \hat{V}_{+}^{(0)}(\mathcal{T}, z, \sigma) \equiv \exp \left\{-\mathcal{G}^{0 \mu ; 0 \nu}(\sigma) \sum_{m \geq 1} \hat{J}_{0 \mu}(m) \frac{z^{-m}}{m} \mathcal{T}_{0 \nu}\right\}, \quad\left[\hat{V}_{+}^{(0)}(\mathcal{T}, z, \sigma), \hat{V}_{+}(\mathcal{T}, z, \sigma)\right]=0
\end{aligned}
$$

where we have collected all $z_{0}$ dependence in the quantity $\hat{G}_{+}\left(\mathcal{T}, z_{0}, \sigma\right)$

$$
\begin{gathered}
\hat{G}_{+}\left(\mathcal{T}, z_{0}, \sigma\right) \equiv \hat{V}_{-}\left(\mathcal{T}, z_{0}, \sigma\right)^{-1} \hat{g}_{+}\left(\mathcal{T}, z_{0}, \sigma\right) z_{0}^{-\hat{F}(\mathcal{T}, \sigma)} \hat{V}_{+}^{(0)}\left(\mathcal{T}, z_{0}, \sigma\right)^{-1} \hat{V}_{+}\left(\mathcal{T}, z_{0}, \sigma\right)^{-1} \\
\hat{F}(\mathcal{T}, \sigma) \equiv-\mathcal{G}^{n(r) \mu ;-n(r), \nu}(\sigma) \frac{\bar{n}(r)}{\rho(\sigma)} \mathcal{T}_{n(r) \mu} \mathcal{T}_{-n(r), \nu}+\mathcal{G}^{0 \mu ; 0 \nu}(\sigma) \hat{J}_{0 \mu}(0) \mathcal{T}_{0 \nu} \\
{\left[\hat{F}(\mathcal{T}, \sigma), \hat{V}_{-}(\mathcal{T}, z, \sigma)\right]=\left[\hat{F}(\mathcal{T}, \sigma), \hat{V}_{+}^{(0)}(\mathcal{T}, z, \sigma)\right]=\left[\hat{F}(\mathcal{T}, \sigma), \hat{V}_{+}(\mathcal{T}, z, \sigma)\right]=0}
\end{gathered}
$$

Following Ref. [12], we observe that $\hat{G}_{+}\left(\mathcal{T}, z_{0}, \sigma\right)$ is in fact independent of the reference point

$$
\partial_{z_{0}} \hat{G}_{+}\left(\mathcal{T}, z_{0}, \sigma\right)=0 \quad \rightarrow \quad \hat{G}_{+}\left(\mathcal{T}, z_{0}, \sigma\right)=\hat{G}_{+}(\mathcal{T}, \sigma)
$$

To verify this one uses (2.20), the explicit forms of $\hat{V}_{ \pm}, \hat{V}_{+}^{(0)}$ and the equation

$$
\begin{aligned}
\partial_{z_{0}} \hat{g}_{+}\left(\mathcal{T}, z_{0}, \sigma\right)=\mathcal{G}^{n(r) \mu ;-n(r), \nu}(\sigma)( & : \hat{J}_{n(r) \mu}\left(z_{0}\right) \hat{g}_{+}\left(\mathcal{T}, z_{0}, \sigma\right):_{M} \\
& \left.-\frac{\bar{n}(r)}{\rho(\sigma)} \frac{1}{z_{0}} \hat{g}_{+}\left(\mathcal{T}, z_{0}, \sigma\right) \mathcal{T}_{n(r) \mu}\right) \mathcal{T}_{-n(r), \nu}
\end{aligned}
$$

which is the twisted vertex operator equation (2.16a) evaluated at the reference point.

The next step is to invert Eq. (2.19a), which allows us to express the quantity $\hat{G}_{+}(\mathcal{T}, \sigma)$ in terms of the twisted vertex operator $\hat{g}_{+}(\mathcal{T}, z, \sigma)$

$$
\hat{G}_{+}(\mathcal{T}, \sigma) \equiv \hat{V}_{-}(\mathcal{T}, z, \sigma)^{-1} \hat{g}_{+}(\mathcal{T}, z, \sigma) \hat{V}_{+}^{(0)}(\mathcal{T}, z, \sigma)^{-1} \hat{V}_{+}(\mathcal{T}, z, \sigma)^{-1} z^{-\hat{F}(\mathcal{T}, \sigma)}
$$


Using the twisted vertex operator equation (2.16a), it can be checked that the right side of (2.23) is independent of $z$ as it should be.

Continuing to follow the strategy of Ref. [12], we note that the relation (2.23) allows us to compute the commutator of the twisted current modes with the quantity $\hat{G}_{+}(\mathcal{T}, \sigma)$. In particular, the twisted current algebra (2.7d) as well as the relations

$$
\begin{gathered}
{\left[\hat{J}_{\bar{n}(r) \mu}\left(m+\frac{\bar{n}(r)}{\rho(\sigma)}\right), \hat{g}_{+}(\mathcal{T}, z, \sigma)\right]=\hat{g}_{+}(\mathcal{T}, z, \sigma) \mathcal{T}_{\bar{n}(r) \mu} z^{m+\frac{\bar{n}(r)}{\rho(\sigma)}}} \\
\sum_{\delta} \mathcal{G}_{n(r) \mu ;-n(r), \delta} \mathcal{G}^{-n(r), \delta ; n(r) \nu}=\sum_{s, \delta} \mathcal{G}_{n(r) \mu ; n(s) \delta} \mathcal{G}^{n(s) \delta ; n(r) \nu}=\delta_{\mu}{ }^{\nu}, \quad \forall n(r), \mu
\end{gathered}
$$

are needed for this computation, and the result

$$
\left[\hat{J}_{\bar{n}(r) \mu}\left(m+\frac{\bar{n}(r)}{\rho(\sigma)}\right), \hat{G}_{+}(\mathcal{T}, \sigma)\right]=\hat{G}_{+}(\mathcal{T}, \sigma) \delta_{m, 0} \delta_{\bar{n}(r), 0} \mathcal{T}_{0 \mu}
$$

is obtained after some algebra.

The algebra (2.25) can in fact be solved to obtain the explicit form of the quantity $\hat{G}_{+}(\mathcal{T}, \sigma)$

$$
\begin{gathered}
\hat{G}_{+}(\mathcal{T}, \sigma)=\hat{\Gamma}\left(\mathcal{T}, \hat{J}_{0}(0), \sigma\right) e^{i \hat{q}^{\mu}(\sigma) \mathcal{T}_{0 \mu}} \\
{\left[\hat{q}^{\mu}(\sigma), \hat{J}_{\bar{n}(s) \nu}\left(n+\frac{\bar{n}(s)}{\rho(\sigma)}\right)\right]=i \delta_{\nu}^{\mu} \delta_{n, 0} \delta_{\bar{n}(s), 0}, \quad\left[\hat{q}^{\mu}(\sigma), \hat{q}^{\nu}(\sigma)\right]=0} \\
{\left[\hat{\Gamma}\left(\mathcal{T}, \hat{J}_{0}(0), \sigma\right), \mathcal{T}_{n(r) \mu}\right]=\left[\hat{\Gamma}\left(\mathcal{T}, \hat{J}_{0}(0), \sigma\right), \hat{J}_{n(s) \nu}\left(n+\frac{n(s)}{\rho(\sigma)}\right)\right]=0}
\end{gathered}
$$

where $\hat{q}^{\mu}(\sigma)$ is a "coordinate" conjugate to the "momentum" $\hat{J}_{0 \mu}(0)$, and $\hat{\Gamma}$ is a sector-dependent Klein transformation (cocycle) which is a so-far undetermined function of $\mathcal{T}_{n(r) \mu}$ and $\hat{J}_{0 \mu}(0)$.

The periodic forms of Eqs. (2.26b) and (2.25) are

$$
\begin{gathered}
{\left[\hat{q}^{\mu}(\sigma), \hat{J}_{n(s) \nu}\left(n+\frac{n(s)}{\rho(\sigma)}\right)\right]=i \delta_{\nu}^{\mu} \delta_{n+\frac{n(s)}{\rho(\sigma)}, 0}} \\
{\left[\hat{J}_{n(r) \mu}\left(m+\frac{n(r)}{\rho(\sigma)}\right), \hat{G}_{+}(\mathcal{T}, \sigma)\right]=\hat{G}_{+}(\mathcal{T}, \sigma) \delta_{m+\frac{n(r)}{\rho(\sigma)}, 0} \mathcal{T}_{n(r) \mu}}
\end{gathered}
$$

where (2.27b follows from $(2.27 \mathrm{a}),(2.26 \mathrm{a})$ and the periodicity $(2.14 \mathrm{~h})$ of the twisted representation matrices. The relations $(2.27 \mathrm{a})$ and $(2.27 \mathrm{~b})$ are consistent with the periodicity $(2.7 \mathrm{e})$ of the twisted current modes, and reduce to (2.26b) and (2.25) in the fundamental range $n \rightarrow \bar{n}$.

\subsection{Summary of the twisted left-mover vertex operators}

Assembling our results above, we summarize our solution for the twisted vertex operators $\left.\left\{\hat{g}_{+}(\mathcal{T}(T, \sigma), z, \sigma), \forall T\right)\right\}$ in sector $\sigma$ of $A_{\text {Cartan } g}(H) / H$

$$
\begin{aligned}
\hat{g}_{+}(\mathcal{T}, z, \sigma)= & z^{-\mathcal{G}^{n(r) \mu ;-n(r), \nu}(\sigma) \frac{\bar{n}(r)}{\rho(\sigma)} \mathcal{T}_{n(r) \mu} \mathcal{T}_{-n(r), \nu}} \hat{\Gamma}\left(\mathcal{T}, \hat{J}_{0}(0), \sigma\right) e^{i \hat{q}^{\mu}(\sigma) \mathcal{T}_{0 \mu}} \\
& \times z^{{\hat{J_{0 \mu}}}(0) \mathcal{G}^{0 \mu ; 0 \nu}(\sigma) \mathcal{T}_{0 \nu}} \hat{V}_{-}(\mathcal{T}, z, \sigma) \hat{V}_{+}^{(0)}(\mathcal{T}, z, \sigma) \hat{V}_{+}(\mathcal{T}, z, \sigma)
\end{aligned}
$$




$$
\begin{gathered}
\hat{V}_{-}(\mathcal{T}, z, \sigma) \equiv \exp \left\{-\mathcal{G}^{n(r) \mu ;-n(r), \nu}(\sigma) \sum_{m \leq-1} \hat{J}_{\bar{n}(r) \mu}\left(m+\frac{\bar{n}(r)}{\rho(\sigma)}\right) \frac{z^{-\left(m+\frac{\bar{n}(r)}{\rho(\sigma)}\right)}}{m+\frac{\bar{n}(r)}{\rho(\sigma)}} \mathcal{T}_{-n(r), \nu}\right\} \\
\hat{V}_{+}^{(0)}(\mathcal{T}, z, \sigma) \equiv \exp \left\{-\mathcal{G}^{0 \mu ; 0 \nu}(\sigma) \sum_{m \geq 1} \hat{J}_{0 \mu}(m) \frac{z^{-m}}{m} \mathcal{T}_{0 \nu}\right\} \\
\hat{V}_{+}(\mathcal{T}, z, \sigma) \equiv \exp \left\{-\mathcal{G}^{n(r) \mu ;-n(r), \nu}(\sigma)\left(1-\delta_{\bar{n}(r), 0}\right) \sum_{m \geq 0} \hat{J}_{\bar{n}(r) \mu}\left(m+\frac{\bar{n}(r)}{\rho(\sigma)}\right) \frac{z^{-\left(m+\frac{\bar{n}(r)}{\rho(\sigma)}\right)}}{m+\frac{\bar{n}(r)}{\rho(\sigma)}} \mathcal{T}_{-n(r), \nu}\right\} \\
{\left[\begin{array}{l}
{\left[\hat{J}_{n(r) \mu}\left(m+\frac{n(r)}{\rho(\sigma)}\right), \hat{J}_{n(s) \nu}\left(n+\frac{n(s)}{\rho(\sigma)}\right)\right]=\left(m+\frac{n(r)}{\rho(\sigma)}\right) \delta_{m+n+\frac{n(r)+n(s)}{\rho(\sigma)}, 0} \mathcal{G}_{n(r) \mu ;-n(r), \nu}(\sigma)} \\
{\left[\hat{q}^{\mu}(\sigma), \hat{J}_{n(s) \nu}\left(n+\frac{n(s)}{\rho(\sigma)}\right)\right]=i \delta_{\nu}^{\mu} \delta_{n+\frac{n(s)}{\rho(\sigma)}, 0}}
\end{array}\right.} \\
\left.\sigma \hat{q}^{\mu}(\sigma), \hat{q}^{\nu}(\sigma)\right]=0 \\
(2.28 \mathrm{~d})
\end{gathered}
$$

where $\mathcal{G} .(\sigma), \mathcal{G}(\sigma)$ and the twisted representation matrices $\mathcal{T}=\mathcal{T}(T, \sigma)$ are given respectively in $(2.8 \mathrm{~b}),(2.8 \mathrm{~d})$ and $(2.14)$. The commutators of the twisted current modes and the Virasoro operators with the twisted vertex operators are found in Eq. (2.14). Using this solution, our assumption (2.17) is easily verified.

Another form of the prefactor in (2.28a) is obtained with the relation

$$
\mathcal{G}^{n(r) \mu ;-n(r), \nu}(\sigma) \frac{\bar{n}(r)}{\rho(\sigma)} \mathcal{T}_{n(r) \mu} \mathcal{T}_{-n(r), \nu}=\frac{1}{2} \mathcal{G}^{n(r) \mu ;-n(r), \nu}(\sigma) \mathcal{T}_{n(r) \mu} \mathcal{T}_{-n(r), \nu}\left(1-\delta_{\bar{n}(r), 0}\right)
$$

which is the abelian limit of the consistency relation (6.20a) in Ref. 10].

Twisted vertex operators are very old [13, 14] and twisted vertex operators related to ours have been studied in mathematics (see e.g. Ref. [15, 16]) and physics (see e.g. Ref. [1]). We emphasize however that our solution (2.28) tells us exactly which twisted vertex operators $\left\{\hat{g}_{+}(\mathcal{T}(T, \sigma), z, \sigma), \forall T\right\}$ appear in each sector $\sigma$ of all the abelian orbifolds in our class.

\subsection{Properties of the twisted vertex operators}

The twisted vertex operators (2.28) of $A_{\text {Cartan } g}(H) / H$ satisfy the following properties.

\section{- Untwisted vertex operators}

In the untwisted sector $\sigma=0$ of each orbifold we know that

$$
\begin{aligned}
& U(0)=U(T, 0)=1, \bar{n}=0, \quad \chi(0)=1 \\
& \hat{J}=J, \quad \mathcal{G}=G, \quad \mathcal{T}=T, \quad \hat{\Gamma}=\Gamma, \quad \hat{q}=q, \quad \hat{g}_{+}=g_{+}
\end{aligned}
$$


and so the twisted result (2.28) reduces to the Fubini-Veneziano vertex operator [17

$$
\begin{gathered}
g_{+}(T, z, 0)=\Gamma(T, J(0)) e^{i q^{a}(0) T_{a}} z^{J_{a}(0) G^{a b} J_{b}(0)} \\
\times \exp \left(G^{a b} \sum_{m \geq 1} J_{a}(-m) \frac{z^{m}}{m} T_{b}\right) \exp \left(-G^{a b} \sum_{m \geq 1} J_{a}(m) \frac{z^{-m}}{m} T_{b}\right) \\
{\left[J_{a}(m), J_{b}(n)\right]=m G_{a b} \delta_{m+n, 0}, \quad\left[q^{a}, J_{b}(n)\right]=i \delta_{a}^{b} \delta_{n, 0}, \quad\left[q^{a}, q^{b}\right]=0} \\
{\left[T_{a}, T_{b}\right]=0, \quad a, b=1 \ldots \operatorname{dim}(\operatorname{Cartan} g)}
\end{gathered}
$$

where the representations $\left\{T_{a}\right\}$ are the Cartan matrices of the ambient Lie algebra $g$.

\section{- Alternate form}

Returning to (2.28), the alternate form of the twisted vertex operators

$$
\begin{aligned}
& \hat{g}_{+}(\mathcal{T}, z, \sigma)= z^{-\mathcal{G}^{n(r) \mu ;-n(r), \nu}(\sigma) \frac{\bar{n}(r)}{\rho(\sigma)} \mathcal{T}_{n(r) \mu} \mathcal{T}_{-n(r), \nu}} \hat{\Gamma}\left(\mathcal{T}, \hat{J}_{0}(0), \sigma\right): e^{i \hat{\beta}^{n(r) \mu}(z, \sigma) \mathcal{T}_{n(r) \mu}:_{M}} \\
& \hat{\beta}^{n(r) \mu}(z, \sigma)=\left\{\hat{q}^{\mu}(\sigma)+\mathcal{G}^{0 \mu ; 0 \nu}(\sigma)\left(\hat{J}_{0 \nu}(0) \ln z+i \sum_{m \neq 0} \hat{J}_{0 \nu}(m) \frac{z^{-m}}{m}\right)\right\} \delta_{\bar{n}(r), 0} \\
&+\left\{i \mathcal{G}^{n(r) \mu ;-n(r), \nu}(\sigma) \sum_{m \in \mathbb{Z}} \hat{J}_{-n(r), \nu}\left(m+\frac{\overline{-n(r)}}{\rho(\sigma)}\right) \frac{z^{-\left(m+\frac{\overline{-n(r)}}{\rho(\sigma)}\right)}}{m+\frac{\overline{-n(r)}}{\rho(\sigma)}}\right\}\left(1-\delta_{\bar{n}(r), 0}\right) \\
& \overline{-n(r)}=\overline{-\bar{n}(r)}=\left\{\begin{array}{l}
\rho(\sigma)-\bar{n}(r) \text { when } \bar{n}(r) \neq 0 \\
0
\end{array}\right.
\end{aligned}
$$

defines a twisted Fubini-Veneziano field $\hat{\beta}$ for each sector $\sigma$ of $A_{\text {Cartan } g}(H) / H$. The mode ordering $M$ defined here in (2.32a) is the left to right ordering $\{\hat{q}, \hat{J}(0),-,+\}$ given explicitly in (2.28a).

\section{- Intrinsic monodromy}

For general WZW models [12] and WZW orbifolds [10] the intrinsic monodromy of the twisted affine primary fields is quite complicated, but this property is easily computable in our abelian limit. From Eqs. (2.28) or (2.32) we find the intrinsic monodromy of the twisted vertex operators

$$
\begin{aligned}
\hat{g}_{+}\left(\mathcal{T}, z e^{2 \pi i}, \sigma\right)= & E(T, \sigma) \hat{g}_{+}(\mathcal{T}, z, \sigma) E(T, \sigma)^{*} \\
& \times e^{2 \pi i\left(-\mathcal{G}^{n(r) \mu ;-n(r), \nu}(\sigma) \frac{\bar{n}(r)}{\rho(\sigma)} \mathcal{T}_{n(r) \mu} \mathcal{T}_{-n(r), \nu}+\hat{J}_{0 \mu}(0) \mathcal{G}^{0 \mu ; 0 \nu}(\sigma) \mathcal{T}_{0 \nu}\right)} \\
E(T, \sigma)_{N(r) \mu}{ }^{N(s) \nu}= & \delta_{N(r) \mu}{ }^{N(s) \nu} E_{N(r)}(T, \sigma), \quad E_{N(r)}(T, \sigma)=e^{-2 \pi i \frac{N(r)}{R(\sigma)}} .
\end{aligned}
$$

To obtain this result, we used the solution (2.14t) of the $\mathcal{T}$-selection rule in the form

$$
e^{-2 \pi i \frac{n(r)}{\rho(\sigma)}} \mathcal{T}_{-n(r), \nu}(T, \sigma)=E(T, \sigma) \mathcal{T}_{-n(r), \nu}(T, \sigma) E(T, \sigma)^{*}
$$




$$
\begin{gathered}
\mathcal{T}_{0 \mu}(T, \sigma)=E(T, \sigma) \mathcal{T}_{0 \mu}(T, \sigma) E(T, \sigma)^{*} \\
\hat{V}_{ \pm}\left(\mathcal{T}, z e^{2 \pi i}, \sigma\right)=E(T, \sigma) \hat{V}_{ \pm}(\mathcal{T}, z, \sigma) E(T, \sigma)^{*} \\
\hat{V}_{+}^{(0)}\left(\mathcal{T}, z e^{2 \pi i}, \sigma\right)=E(T, \sigma) \hat{V}_{+}^{(0)}(\mathcal{T}, z, \sigma) E(T, \sigma)^{*} \\
{\left[E(T, \sigma), \hat{\Gamma}\left(\mathcal{T}, \hat{J}_{0}(0), \sigma\right)\right]=\left[E(T, \sigma), \hat{G}_{+}(\mathcal{T}, \sigma)\right]=0}
\end{gathered}
$$

where the relations (2.34d, $2.34 \mathrm{~d})$ follow from the special case of the $\mathcal{T}$-selection rule in (2.34b). Similarly we find the intrinsic monodromy

$$
\hat{\beta}^{n(r) \mu}\left(z e^{2 \pi i}, \sigma\right)=\hat{\beta}^{n(r) \mu}(z, \sigma) e^{2 \pi i \frac{n(r)}{\rho(\sigma)}}+\delta_{\bar{n}(r), 0} 2 \pi i \mathcal{G}^{0 \mu ; 0 \nu}(\sigma) \hat{J}_{0 \nu}(0)
$$

of the twisted Fubini-Veneziano field $\hat{\beta}$ in (2.32), and the $\mathcal{T}$-selection rule (2.34a) guarantees the consistency of this monodromy with that given in (2.33) for the twisted vertex operators themselves.

\section{- Braid relations}

The following exchange operations

$$
\begin{gathered}
z^{\hat{J}_{0 \mu}(0) \mathcal{G}^{0 \mu ; 0 \nu}(\sigma) \mathcal{T}_{0 \nu}^{(1)}} e^{i \hat{q}^{\mu}(\sigma) \mathcal{T}_{0 \mu}^{(2)}}=e^{i \hat{q}^{\mu}(\sigma) \mathcal{T}_{0 \mu}^{(2)}} z^{\hat{J}_{0 \mu}(0) \mathcal{G}^{0 \mu ; 0 \nu}(\sigma) \mathcal{T}_{0 \nu}^{(1)}} z^{\mathcal{T}_{0 \mu}^{(2)}} \mathcal{G}^{0 \mu ; 0 \nu}(\sigma) \mathcal{T}_{0 \nu}^{(1)} \\
\hat{V}_{+}^{(0)}\left(\mathcal{T}^{(1)}, z_{1}, \sigma\right) \hat{V}_{-}\left(\mathcal{T}^{(2)}, z_{2}, \sigma\right)=\hat{V}_{-}\left(\mathcal{T}^{(2)}, z_{2}, \sigma\right) \hat{V}_{+}^{(0)}\left(\mathcal{T}^{(1)}, z_{1}, \sigma\right)\left(1-\frac{z_{0 \mu}^{(2)} \mathcal{G}^{0 \mu ; 0 \nu}(\sigma) \mathcal{T}_{0 \nu}^{(1)}}{z_{1}}\right. \\
\hat{V}_{+}\left(\mathcal{T}^{(1)}, z_{1}, \sigma\right) \hat{V}_{-}\left(\mathcal{T}^{(2)}, z_{2}, \sigma\right)=\hat{V}_{-}\left(\mathcal{T}^{(2)}, z_{2}, \sigma\right) \hat{V}_{+}\left(\mathcal{T}^{(1)}, z_{1}, \sigma\right) \\
\times \exp \left\{\mathcal{T}_{n(r) \mu}^{(2)} \mathcal{G}^{n(r) \mu ;-n(r), \nu}(\sigma) \mathcal{T}_{-n(r), \nu}^{(1)}\left(1-\delta_{\bar{n}(r), 0}\right) I_{\frac{\bar{n}(r)}{\rho(\sigma)}}\left(\frac{z_{1}}{z_{2}}, \infty\right)\right\} \\
I_{\frac{\bar{n}(r)}{\rho(\sigma)}}(y, \infty) \equiv \int_{\infty}^{y} \frac{d x}{x-1} x^{-\frac{\bar{n}(r)}{\rho(\sigma)}}=-\sum_{n=0}^{\infty} \frac{y^{-\left(n+\frac{\bar{n}(r)}{\rho(\sigma)}\right)}}{n+\frac{\bar{n}(r)}{\rho(\sigma)}}, \quad|y|>1
\end{gathered}
$$

hold for $\left|z_{1}\right|>\left|z_{2}\right|$ and $\sigma=0, \ldots, N_{c}-1$. The integrals $I_{\bar{n}(r) / \rho(\sigma)}$ were encountered in Ref. [10. and evaluated as indefinite integrals in the last appendix of that reference.

\section{- Operator products}

Using (2.36) we find that the exact operator products of the twisted vertex operators for $\hat{\Gamma}=1$

$$
\begin{aligned}
& \hat{g}_{+}\left(\mathcal{T}^{(1)}, z_{1}, \sigma\right) \hat{g}_{+}\left(\mathcal{T}^{(2)}, z_{2}, \sigma\right)=: \hat{g}_{+}\left(\mathcal{T}^{(1)}, z_{1}, \sigma\right) \hat{g}_{+}\left(\mathcal{T}^{(2)}, z_{2}, \sigma\right):_{M} B\left(\mathcal{T}^{(1)}, \mathcal{T}^{(2)} ; z_{1}, z_{2}\right) \\
& B\left(\mathcal{T}^{(1)}, \mathcal{T}^{(2)} ; z_{1}, z_{2}\right) \\
& \equiv z_{12}^{(2)} \mathcal{G}^{0 \mu ; 0 \nu}(\sigma) \mathcal{T}_{0 \nu}^{(1)} \exp \left\{\mathcal{T}_{n(r) \mu}^{(2)} \mathcal{G}^{n(r) \mu ;-n(r), \nu}(\sigma) \mathcal{T}_{-n(r), \nu}^{(1)}\left(1-\delta_{\bar{n}(r), 0}\right) I_{\frac{\bar{n}(r)}{\rho(\sigma)}}\left(\frac{z_{1}}{z_{2}}, \infty\right)\right\} \\
&: \hat{g}_{+}\left(\mathcal{T}^{(1)}, z_{1}, \sigma\right) \hat{g}_{+}\left(\mathcal{T}^{(2)}, z_{2}, \sigma\right):_{M} \\
& \equiv e^{i \hat{q}^{\mu}(\sigma)\left(\mathcal{T}_{0 \mu}^{(1)}+\mathcal{T}_{0 \mu}^{(2)}\right)} z_{1}^{\hat{F}\left(\mathcal{T}^{(1)}, \sigma\right)} z_{2}^{\hat{F}\left(\mathcal{T}^{(2)}, \sigma\right)} \hat{V}_{-}\left(\mathcal{T}^{(1)}, z_{1}, \sigma\right) \hat{V}_{-}\left(\mathcal{T}^{(2)}, z_{2}, \sigma\right) \\
& \quad \times \hat{V}_{+}^{(0)}\left(\mathcal{T}^{(1)}, z_{1}, \sigma\right) \hat{V}_{+}^{(0)}\left(\mathcal{T}^{(2)}, z_{2}, \sigma\right) \hat{V}_{+}\left(\mathcal{T}^{(1)}, z_{1}, \sigma\right) \hat{V}_{+}\left(\mathcal{T}^{(2)}, z_{2}, \sigma\right)
\end{aligned}
$$

also hold for $\left|z_{1}\right|>\left|z_{2}\right|, \sigma=0, \ldots N_{c}-1$. The quantity $\hat{F}(\mathcal{T}, \sigma)$ is defined in (2.20b). 


\subsection{Correlators of the twisted vertex operators}

The ground state conditions in Eq. (2.11) imply the further conditions on the partial currents and the components of the twisted vertex operators

$$
\begin{gathered}
\hat{J}_{n(r) \mu}^{+}(z)|0\rangle_{\sigma}={ }_{\sigma}\langle 0| \hat{J}_{n(r) \mu}^{-}(z)=0 \\
\hat{V}_{+}^{(0)}(\mathcal{T}, z, \sigma)|0\rangle_{\sigma}=\hat{V}_{+}(\mathcal{T}, z, \sigma)|0\rangle_{\sigma}=|0\rangle_{\sigma}, \quad{ }_{\sigma}\langle 0| \hat{V}_{-}(\mathcal{T}, z, \sigma)={ }_{\sigma}\langle 0|
\end{gathered}
$$

and these conditions allow us to evaluate the correlators in sector $\sigma$ of $A_{\text {Cartan } g}(H) / H$ :

$$
\begin{aligned}
& \hat{A}_{+}(\mathcal{T}, z, \sigma) \equiv\left\langle\hat{g}_{+}\left(\mathcal{T}^{(1)}, z_{1}, \sigma\right) \cdots \hat{g}_{+}\left(\mathcal{T}^{(N)}, z_{N}, \sigma\right)\right\rangle_{\sigma}, \quad \sigma=0, \ldots, N_{c}-1 \\
& =C_{+}(\mathcal{T}, \sigma)\left(\prod_{\rho} z_{\rho}^{-\mathcal{G}^{n(r) \mu ;-n(r), \nu}(\sigma) \frac{\bar{n}(r)}{\rho(\sigma)} \mathcal{T}_{n(r) \mu}^{(\rho)} \mathcal{T}_{-n(r), \nu}^{(\rho)}}\right)\left(\prod_{\rho<\kappa} z_{\rho \kappa}^{\mathcal{T}_{\rho \mu}^{(\kappa)} \mathcal{G}^{0 \mu ; 0 \nu}(\sigma) \mathcal{T}_{0 \nu}^{(\rho)}}\right) \\
& \times \prod_{\rho<\kappa} \exp \left\{\mathcal{T}_{n(r) \mu}^{(\kappa)} \mathcal{G}^{n(r) \mu ;-n(r), \nu}(\sigma) \mathcal{T}_{-n(r), \nu}^{(\rho)}\left(1-\delta_{\bar{n}(r), 0}\right) I_{\frac{\bar{n}(r)}{\rho(\sigma)}}\left(\frac{z_{\rho}}{z_{\kappa}}, \infty\right)\right\} \\
& C_{+}(\mathcal{T}, \sigma) \equiv\left\langle\hat{G}_{+}\left(\mathcal{T}^{(1)}, \sigma\right) \cdots \hat{G}_{+}\left(\mathcal{T}^{(N)}, \sigma\right)\right\rangle_{\sigma} \\
& \left\langle\left[\hat{J}_{0 \mu}(0), \hat{G}_{+}\left(\mathcal{T}^{(1)}, \sigma\right) \cdots \hat{G}_{+}\left(\mathcal{T}^{(N)}, \sigma\right)\right]\right\rangle_{\sigma}=0 \quad \Rightarrow \quad C_{+}(\mathcal{T}, \sigma)\left(\sum_{\rho=1}^{N} \mathcal{T}_{0 \mu}^{(\rho)}\right)=0, \quad \forall \mu
\end{aligned}
$$

Here we have used the residual symmetry generators $\hat{J}_{0 \mu}(0)$ to include the global Ward identities in $(2.39 \mathrm{~d})$. Note that the global Ward identities can also be written as

$$
\left(\sum_{\rho=1}^{N} \mathcal{T}_{0 \mu}^{(\rho)}\right) C_{+}(\mathcal{T}, \sigma)=0, \quad \forall \mu
$$

because the twisted representation matrices commute with $\hat{g}_{+}$. Similarly, the constant factor $C_{+}(\mathcal{T}, \sigma)$ can be moved to the right in $(2.39 \mathrm{~b})$.

Another relation on the constant factor $C_{+}(\mathcal{T}, \sigma)$ follows from $\left.2.34 \mathrm{e}\right)$

$$
\begin{gathered}
{\left[E(\{T\}, \sigma), \hat{G}_{+}\left(\mathcal{T}^{(1)}, \sigma\right) \cdots \hat{G}_{+}\left(\mathcal{T}^{(N)}, \sigma\right)\right]=0 \rightarrow\left[E(\{T\}, \sigma), C_{+}(\mathcal{T}, \sigma)\right]=0} \\
E(\{T\}, \sigma) \equiv \otimes_{\rho=1}^{N} E\left(T^{(\rho)}, \sigma\right)
\end{gathered}
$$

where the eigenvalue matrix $E(T, \sigma)$ is given in $(2.33 \mathrm{~b})$.

We finally note that the orbifold correlators (2.39) satisfy the twisted $\mathrm{KZ}$-like equations

$$
\partial_{\kappa} \hat{A}_{+}(\mathcal{T}, z, \sigma)=\hat{A}_{+}(\mathcal{T}, z, \sigma) \hat{W}_{\kappa}(\mathcal{T}, z, \sigma), \quad \sigma=0, \ldots, N_{c}-1
$$




$$
\begin{aligned}
& \hat{W}_{\kappa}(\mathcal{T}, z, \sigma)=\mathcal{G}^{n(r) \mu ;-n(r), \nu}(\sigma)\left[\sum_{\rho \neq \kappa}\left(\frac{z_{\rho}}{z_{\kappa}}\right)^{\frac{\bar{n}(r)}{\rho(\sigma)}} \frac{1}{z_{\kappa \rho}} \mathcal{T}_{n(r) \mu}^{(\rho)} \mathcal{T}_{-n(r), \nu}^{(\kappa)}-\frac{\bar{n}(r)}{\rho(\sigma)} \frac{1}{z_{\kappa}} \mathcal{T}_{n(r) \mu}^{(\kappa)} \mathcal{T}_{-n(r), \nu}^{(\kappa)}\right]
\end{aligned}
$$

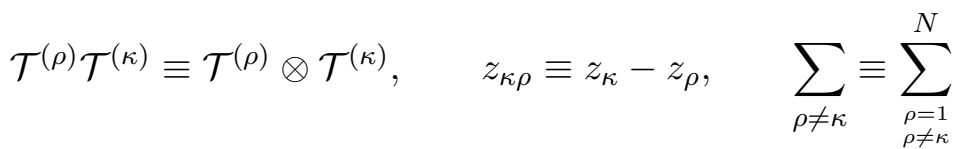

as they must because these equations follow directly from the twisted vertex operator equation (2.16a) and the ground state condition (2.11).

This completes our general discussion of the left-mover sectors of the abelian orbifolds $A_{\text {Cartan } g}(H) / H$, and we turn in the following sections to specific examples in $A_{\text {Cartan } g}(H) / H$. The right-mover sectors of $A_{\text {Cartan } g}(H) / H$ are considered in Subsecs. 2.9 and 2.10 .

\subsection{Example: Abelian permutation orbifolds}

In this subsection we study the abelian permutation orbifolds

$$
\frac{A_{\text {Cartan } g}(H)}{H}, \quad H(\text { permutation }) \subset \operatorname{Aut}(\operatorname{Cartan} g)
$$

as a special case of our large example $A_{\text {Cartan } g}(H) / H$. In the permutation-symmetric theory $A_{\text {Cartan } g}(H)$, the permutations act among the copies Cartan $\mathfrak{g}^{I}$ of Cartan $\mathfrak{g}$

$$
\begin{gathered}
\text { Cartan } g=\oplus_{I=0}^{K-1} \operatorname{Cartan}_{\mathfrak{g}^{I},} \quad \text { Cartan } \mathfrak{g}^{I} \simeq \text { Cartan } \mathfrak{g}, \quad K \leq N \\
H(\text { permutation }) \subset S_{N}, \quad k_{I}=k, \quad x_{I}=x, \quad \eta_{a(I) b(I)}^{I}=\eta_{a b}, \quad T^{I} \simeq T \\
{\left[T_{a}, T_{b}\right]=0, \quad a, b=1 \ldots \operatorname{dim}(\text { Cartan } \mathfrak{g})}
\end{gathered}
$$

where $T_{a}$ are the Cartan matrices of Cartan $\mathfrak{g} \subset \mathfrak{g}$.

For the WZW permutation orbifolds it is conventional [4, 10] to use the notation $\mu=a j$, $a=1 \ldots \operatorname{dim} \mathfrak{g}$ for the degeneracy indices of the $H$-eigenvalue problem, and it is known [10] for the extended $H$-eigenvalue problem (2.15) that $R(\sigma)=\rho(\sigma)$ and $N(r)=n(r)$. For the abelian permutation orbifolds we know that $\mathfrak{g} \rightarrow$ Cartan $\mathfrak{g}$ so the corresponding relabelling for the abelian permutation orbifolds is

$$
n(r) \mu \rightarrow n(r) a j, \quad N(r) \mu \rightarrow n(r) \alpha j, \quad a=1 \ldots \operatorname{dim}(\text { Cartan } \mathfrak{g}), \quad \alpha=1 \ldots \operatorname{dim} T
$$

and then the explicit form of all the required duality transformations can be read off as the abelian limit of those given for the WZW permutation orbifolds in Ref. [10]. It will be helpful here to introduce the further spectral index relabelling $n(r) \rightarrow \hat{j}$

$$
\begin{aligned}
& n(r) a j \rightarrow \hat{j} a j, \quad N(r) a j \quad \rightarrow \quad \hat{j} \alpha j \\
& \frac{N(r)}{R(\sigma)}=\frac{n(r)}{\rho(\sigma)}=\frac{\hat{j}}{f_{j}(\sigma)}, \quad \overline{\hat{j}}=0,1, \ldots, f_{j}(\sigma)-1, \quad \sum_{j} f_{j}(\sigma)=K \leq N
\end{aligned}
$$




$$
\sigma=0, \ldots, N_{c}-1
$$

where the correspondence (2.46b), 2.460) was also given in Ref. [10]. In this notation, each element $h_{\sigma} \in H$ (permutation) is expressed in terms of disjoint cycles $j$ of size and order $f_{j}(\sigma)$, and the hatted index $\hat{j}$ runs inside the disjoint cycle $j$. The disjoint cycles are labelled periodically $\hat{j} \rightarrow \hat{j} \pm f_{j}(\sigma)$ so that $\overline{\hat{j}}$ in 2.46b) is the pullback of $\hat{j}$ to the fundamental range. As an example we recall [4] the data for $H$ (permutation) $=S_{N}$

$$
K=N, \quad f_{j}(\sigma)=\sigma_{j}, \quad \sigma_{j+1} \leq \sigma_{j}, \quad j=0, \ldots, n(\vec{\sigma})-1, \quad \sum_{j=0}^{n(\vec{\sigma})-1} \sigma_{j}=N
$$

and the data for $H$ (permutation) $=\mathbb{Z}_{\lambda}$ is included in Subsec. 2.7.

Then we may read the following results directly from Ref. [10]:

$$
\begin{aligned}
& \hat{J}_{n(r) a j}(z) \rightarrow \hat{J}_{\hat{j} a j}(z), \quad \hat{J}_{\hat{j} a j}\left(z e^{2 \pi i}\right)=e^{-2 \pi i \frac{\hat{j}}{f_{j}(\sigma)}} \hat{J}_{\hat{j} a j}(z), \quad \hat{J}_{\hat{j} \pm f_{j}(\sigma), a j}(z)=\hat{J}_{\hat{j} a j}(z) \\
& \hat{J}_{\hat{j} a j}(z)=\sum_{m \in \mathbb{Z}} \hat{J}_{\hat{j} a j}\left(m+\frac{\hat{j}}{f_{j}(\sigma)}\right) z^{-\left(m+\frac{\hat{j}}{f_{j}(\sigma)}\right)-1}, \quad \hat{J}_{\hat{j} \pm f_{j}(\sigma), a j}\left(m+\frac{\hat{j} \pm f_{j}(\sigma)}{f_{j}(\sigma)}\right)=\hat{J}_{\hat{j} a j}\left(m \pm 1+\frac{\hat{j}}{f_{j}(\sigma)}\right) \\
& \mathcal{G}_{n(r) \mu ; n(s) \nu}(\sigma) \quad \rightarrow \quad \mathcal{G}_{\hat{j} a j ; \hat{l} b l}=\hat{k}_{j}(\sigma) \eta_{a b} \delta_{j l} \delta_{\hat{j}+\hat{l}, 0 \bmod f_{j}(\sigma)}, \quad \hat{k}_{j}(\sigma)=k f_{j}(\sigma) \\
& \mathcal{G}^{n(r) \mu ; n(s) \nu}(\sigma) \quad \rightarrow \quad \mathcal{G}^{\hat{j a j} ; \hat{l} b l}(\sigma)=\frac{\eta^{a b}}{\hat{k}_{j}(\sigma)} \delta^{j l} \delta_{\hat{j}+\hat{l}, 0 \bmod f_{j}(\sigma)} \\
& {\left[\hat{J}_{\hat{j} a j}\left(m+\frac{\hat{j}}{f_{j}(\sigma)}\right), \hat{J}_{\hat{l} b l}\left(n+\frac{\hat{\imath}}{f_{j}(\sigma)}\right)\right]=\delta_{j l} \hat{k}_{j}(\sigma) \eta_{a b}\left(m+\frac{\hat{j}}{f_{j}(\sigma)}\right) \delta_{m+n+\frac{\hat{j}+\hat{l}}{f_{j}(\sigma)}, 0}} \\
& \mathcal{T}_{n(r) \mu}=T_{a} t_{n(r) \mu}(\sigma) \quad \rightarrow \quad \mathcal{T}_{\hat{j} a j}=T_{a} t_{\hat{j} j}(\sigma), \quad\left[\mathcal{T}_{\hat{j} a j}(T, \sigma), \mathcal{T}_{\hat{l} b l}(T, \sigma)\right]=0 \\
& {\left[T_{a}, T_{b}\right]=0, \quad t_{\hat{j} j}(\sigma) t_{\hat{l} l}(\sigma)=\delta_{j l} t_{\hat{j}+\hat{l}, j}(\sigma), \quad t_{\hat{j} \pm f_{j}(\sigma), j}(\sigma)=t_{\hat{j} j}(\sigma)} \\
& t_{\hat{j} j}(\sigma)_{\hat{l} l}^{\hat{m} m}=\delta_{j l} \delta_{l}^{m} \delta_{\hat{j}+\hat{l}-\hat{m}, 0 \bmod f_{j}(\sigma)} \\
& {\left[\hat{J}_{\hat{j} a j}\left(m+\frac{\hat{j}}{f_{j}(\sigma)}\right), \hat{g}_{+}(\mathcal{T}, z, \sigma)\right]=\hat{g}_{+}(\mathcal{T}, z, \sigma) T_{a} t_{\hat{j} j}(\sigma) z^{m+\frac{\hat{j}}{f_{j}(\sigma)}}} \\
& {\left[L_{\sigma}(m), \hat{g}_{+}(\mathcal{T}, z, \sigma)\right]=z^{m}\left(z \partial_{z}+\Delta(\mathcal{T})(m+1)\right) \hat{g}_{+}(\mathcal{T}, z, \sigma), \quad \frac{\left(\eta^{a b} T_{a} T_{b}\right)_{\alpha}{ }^{\beta}}{2 k}=\Delta(T) \delta_{\alpha}^{\beta}}
\end{aligned}
$$




$$
a=1 \ldots \operatorname{dim}(\operatorname{Cartan} \mathfrak{g}), \quad \overline{\hat{j}}=0, \ldots, f_{j}(\sigma)-1, \quad \overline{\hat{l}}=0, \ldots, f_{l}(\sigma)-1
$$

Relative to Ref. [10 the only differences here are the abelian form of the twisted current algebra (2.48 $)$ and the fact that the Cartan matrices $T_{a}$ of $\mathfrak{g}$ commute. The quantity $\Delta(T)$ in $(2.48 \mathrm{j})$ is the conformal weight of untwisted representation $T$ under untwisted Cartan $\mathfrak{g}$. Note that, as in the nonabelian case, $j$ and $l$ are semisimplicity indices for the twisted current algebra. See also App. A, which solves the $H$-eigenvalue problem for all permutation groups in this notation.

The spectral index relabelling helps to simplify many of the sums encountered in our general discussion above. For example, we obtain for the Virasoro operator and ground state conformal weight $\hat{\Delta}_{0}(\sigma)$ of sector $\sigma$

$$
\begin{gathered}
\mathcal{L}^{n(r) a j ; n(s), b l}(\sigma) \rightarrow \mathcal{L}^{\hat{j a j}, \hat{l} b l}(\sigma)=\frac{\eta^{a b} \delta^{j l}}{2 \hat{k}_{j}(\sigma)} \delta_{\hat{j}+\hat{l}, 0 \bmod f_{j}(\sigma)} \\
L_{\sigma}(m)=\frac{1}{2 k} \sum_{j} \sum_{\hat{j}=0}^{f_{j}(\sigma)-1} \frac{1}{f_{j}(\sigma)} \sum_{p \in \mathbb{Z}} \eta^{a b}: \hat{J}_{\hat{j} a j}\left(p+\frac{\hat{j}}{f_{j}(\sigma)}\right) \hat{J}_{-\hat{j}, b j}\left(m-p-\frac{\hat{j}}{f_{j}(\sigma)}\right):_{M}+\delta_{m, 0} \hat{\Delta}_{0}(\sigma) \\
\hat{c}(\sigma)=c=K \operatorname{dim}(\operatorname{Cartan} \mathfrak{g}), \quad \hat{\Delta}_{0}(\sigma)=\frac{\operatorname{dim}(\operatorname{Cartan} \mathfrak{g})}{24} \sum_{j}\left(f_{j}(\sigma)-\frac{1}{f_{j}(\sigma)}\right)
\end{gathered}
$$

where : $\cdot:_{M}$ is mode normal ordering [2, 3, 11, [4, 10]. Here we used $\mu(r)=\mu=a j$ and the identity

$$
\sum_{r, \mu(r)} \frac{\bar{n}(r)}{2 \rho(\sigma)}\left(1-\frac{\bar{n}(r)}{\rho(\sigma)}\right)=\operatorname{dim}(\operatorname{Cartan} \mathfrak{g}) \sum_{j} \sum_{\hat{j}=0}^{f_{j}(\sigma)-1} \frac{\hat{j}}{2 f_{j}(\sigma)}\left(1-\frac{\hat{j}}{f_{j}(\sigma)}\right)
$$

to obtain (2.49d) from (2.13d). The result (2.49c) is the abelian limit of the formula given for each sector of all WZW permutation orbifolds in Refs. [4] and [10].

Similarly, we find that the following identities

$$
\begin{gathered}
\sum_{j} \sum_{\hat{j}=0}^{f_{j}(\sigma)-1} \frac{1}{f_{j}(\sigma)} t_{0 j}(\sigma)=\sum_{j} t_{0 j}(\sigma)=\mathbb{1}, \quad(\mathbb{1})_{\hat{j} j}^{\hat{l} l}=\delta_{j}^{l} \delta_{\hat{j}-\hat{l}, 0 \bmod f_{j}(\sigma)} \\
\mathcal{G}^{n(r) a j ;-n(r) b l}(\sigma) \mathcal{T}_{n(r) a j} \mathcal{T}_{-n(r), b l}=2 \Delta(T) \mathbb{1}, \quad \mathcal{G}^{0 a j ; 0 b l}(\sigma) \mathcal{T}_{0 a j} \mathcal{T}_{0 b l}=2 \Delta(T) \sum_{j} \frac{1}{f_{j}(\sigma)} t_{0 j}(\sigma) \\
\sum_{j} \frac{1}{f_{j}(\sigma)} t_{0 j}(\sigma)_{\hat{l} l}^{\hat{m} m}=\frac{1}{f_{l}(\sigma)}\left(\mathbb{1}_{\hat{l} l}^{\hat{\imath} m}=\left(\mathbb{1}_{\hat{l} l}^{\hat{m} m} \frac{1}{f_{m}(\sigma)}\right.\right.
\end{gathered}
$$




$$
\begin{aligned}
\mathcal{G}^{n(r) a j ;-n(r) b l}(\sigma) \frac{\bar{n}(r)}{\rho(\sigma)} \mathcal{T}_{n(r) a j} \mathcal{T}_{-n(r), b l}= & \frac{1}{2} \mathcal{G}^{n(r) a j ;-n(r) b l}(\sigma) \mathcal{T}_{n(r) a j} \mathcal{T}_{-n(r), b l}\left(1-\delta_{\bar{n}(r), 0}\right) \\
& =\Delta(T)\left(\mathbb{1}-\sum_{j} \frac{1}{f_{j}(\sigma)} t_{0 j}(\sigma)\right)
\end{aligned}
$$

are useful in simplifying the general twisted vertex operators and correlators above. Closely related identities and simplifications are given for the nonabelian case in App. B. There we also include the spectral integer relabelling $n(r) \rightarrow \hat{j}$ of the twisted KZ equations given in Ref. [10] for the WZW permutation orbifolds.

Then we obtain from Eq. (2.28) the explicit form of the twisted vertex operators in each sector $\sigma$ of the abelian permutation orbifolds

$$
\begin{aligned}
& \hat{g}_{+}(\mathcal{T}, z, \sigma)=z^{-\Delta(T)\left(\mathbb{1}-\sum_{j} \frac{t_{0 j}(\sigma)}{f_{j}(\sigma)}\right)} \hat{\Gamma}\left(\mathcal{T}, \hat{J}_{0}(0), \sigma\right) e^{i T_{a} \sum_{j} \hat{q}^{a j}(\sigma) t_{0 j}(\sigma)} z^{\frac{\eta^{a b} T_{b}}{k} \sum_{j} \hat{J}_{0 a j}(0) t_{0 j}(\sigma) / f_{j}(\sigma)} \\
& \times \exp \left(-\frac{\eta^{a b} T_{b}}{k} \sum_{j} \sum_{\hat{j}=0}^{f_{j}(\sigma)-1} \frac{1}{f_{j}(\sigma)} \sum_{m \leq-1} \hat{J}_{\hat{j} a j}\left(m+\frac{\hat{j}}{f_{j}(\sigma)}\right) \frac{z^{-\left(m+\frac{\hat{j}}{f_{j}(\sigma)}\right)}}{m+\frac{\hat{j}}{f_{j}(\sigma)}} t_{-\hat{j}, j}(\sigma)\right) \\
& \times \exp \left(-\frac{\eta^{a b} T_{b}}{k} \sum_{j} \frac{1}{f_{j}(\sigma)} \sum_{m \geq 1} \hat{J}_{0 a j}(m) \frac{z^{-m}}{m} t_{0 j}(\sigma)\right) \\
& \times \exp \left(-\frac{\eta^{a b} T_{b}}{k} \sum_{j} \sum_{\hat{j}=1}^{f_{j}(\sigma)-1} \frac{1}{f_{j}(\sigma)} \sum_{m \geq 0} \hat{J}_{\hat{j} a j}\left(m+\frac{\hat{j}}{f_{j}(\sigma)}\right) \frac{z^{-\left(m+\frac{\hat{j}}{f_{j}(\sigma)}\right)}}{m+\frac{\hat{j}}{f_{j}(\sigma)}} t_{-\hat{j}, j}(\sigma)\right) \\
& {\left[\hat{q}^{a j}(\sigma), \hat{J}_{\hat{l} b l}\left(m+\frac{\hat{l}}{f_{l}(\sigma)}\right)\right]=i \delta_{l}^{j} \delta_{b}^{a} \delta_{m+\frac{\hat{l}}{f_{l}(\sigma)}, 0}, \quad\left[\hat{q}^{a j}(\sigma), \hat{q}^{b l}(\sigma)\right]=0} \\
& {\left[\hat{J}_{\hat{j} a j}\left(m+\frac{\hat{j}}{f_{j}(\sigma)}\right), \hat{J}_{\hat{l} b l}\left(n+\frac{\hat{l}}{f_{j}(\sigma)}\right)\right]=\delta_{j l} k f_{j}(\sigma) \eta_{a b}\left(m+\frac{\hat{j}}{f_{j}(\sigma)}\right) \delta_{m+n+\frac{\hat{j}+\hat{l}}{f_{j}(\sigma)}, 0}} \\
& a=1 \ldots \operatorname{dim}(\operatorname{Cartan} \mathfrak{g}), \quad \overline{\hat{j}}=0, \ldots f_{j}(\sigma)-1, \quad \sum_{j} f_{j}(\sigma)=K \leq N \\
& \sigma=0, \ldots, N_{c}-1
\end{aligned}
$$

The commutator of the twisted current modes with the twisted vertex operators is given in Eq. (2.488). The twisted vertex operators (2.52) can be written in a $j$-factorized form

$$
\hat{g}_{+}(\mathcal{T}, z, \sigma)=\hat{\Gamma}\left(\mathcal{T}, \hat{J}_{0}(0), \sigma\right) \prod_{j} \hat{V}_{j}(\mathcal{T}, z, \sigma)
$$




$$
\begin{aligned}
\hat{V}_{j}(\mathcal{T}, z, \sigma) \equiv & z^{-\Delta(T)\left(1-\frac{1}{f_{j}(\sigma)}\right) t_{0 j}(\sigma)} e^{i T_{a} \hat{q}^{a j}(\sigma) t_{0 j}(\sigma)} z^{\frac{\eta^{a b} T_{b}}{k f_{j}(\sigma)} \hat{J}_{0 a j}(0) t_{0 j}(\sigma)} \\
& \times \exp \left(-\frac{\eta^{a b} T_{b}}{k f_{j}(\sigma)} \sum_{\hat{j}=0}^{f_{j}(\sigma)-1} \sum_{m \leq-1} \hat{J}_{\hat{j} a j}\left(m+\frac{\hat{j}}{f_{j}(\sigma)}\right) \frac{z^{-\left(m+\frac{\hat{j}}{f_{j}(\sigma)}\right)}}{m+\frac{\hat{j}}{f_{j}(\sigma)}} t_{-\hat{j}, j}(\sigma)\right) \\
& \times \exp \left(-\frac{\eta^{a b} T_{b}}{k f_{j}(\sigma)} \sum_{m \geq 1} \hat{J}_{0 a j}(m) \frac{z^{-m}}{m} t_{0 j}(\sigma)\right) \\
& \times \exp \left(-\frac{\eta^{a b} T_{b}}{k f_{j}(\sigma)} \sum_{\hat{j}=1}^{f_{j}(\sigma)-1} \sum_{m \geq 0} \hat{J}_{\hat{j} a j}\left(m+\frac{\hat{j}}{f_{j}(\sigma)}\right) \frac{z^{-\left(m+\frac{\hat{j}}{f_{j}(\sigma)}\right)}}{m+\frac{\hat{j}}{f_{j}(\sigma)}} t_{-\hat{j}, j}(\sigma)\right)
\end{aligned}
$$

$$
\left[\hat{V}_{j}(\mathcal{T}, z, \sigma), \hat{V}_{l}(\mathcal{T}, z, \sigma)\right]=0 \quad \text { when } j \neq l
$$

which reflects the semisimplicity of the abelian orbifold affine algebra (2.52c).

Using (2.37) and (2.51), we also give the explicit form of the operator product of these twisted vertex operators at $\hat{\Gamma}=1$

$$
\begin{aligned}
\hat{g}_{+}\left(\mathcal{T}^{(1)}, z_{1}, \sigma\right) \hat{g}_{+}\left(\mathcal{T}^{(2)}, z_{2}, \sigma\right) & =: \hat{g}_{+}\left(\mathcal{T}^{(1)}, z_{1}, \sigma\right) \hat{g}_{+}\left(\mathcal{T}^{(2)}, z_{2}, \sigma\right):_{M} \\
& \times z_{12}^{\frac{T_{a}^{(2)} \eta_{a b} T_{b}^{(1)}}{k}} \sum_{j} \frac{1}{f_{j}(\sigma)} t_{0 j}^{(2)}(\sigma) t_{0 j}^{(1)}(\sigma) \\
& \times \exp \left\{\frac{T_{a}^{(2)} \eta_{a b} T_{b}^{(1)}}{k} \sum_{j} \sum_{\hat{j}=1}^{f_{j}(\sigma)-1} \frac{t_{\hat{j} j}^{(2)}(\sigma) t_{-\hat{j}, j}^{(1)}(\sigma)}{f_{j}(\sigma)} I_{\frac{\hat{j}}{f_{j}(\sigma)}}\left(\frac{z_{1}}{z_{2}}, \infty\right)\right\}
\end{aligned}
$$

where the integrals $I_{\bar{n}(r) / \rho(\sigma)}$ are defined in Eq. (2.36d). Similarly, we obtain the explicit form of the twisted left-mover correlators

$$
\begin{aligned}
& \hat{A}_{+}(\mathcal{T}, z, \sigma) \equiv\left\langle\hat{g}_{+}\left(\mathcal{T}^{(1)}, z_{1}, \sigma\right) \cdots \hat{g}_{+}\left(\mathcal{T}^{(N)}, z_{N}, \sigma\right)\right\rangle_{\sigma} \\
& =C_{+}(\mathcal{T}, \sigma)\left(\prod_{\rho} z_{\rho}^{-\Delta\left(T^{(\rho)}\right)\left(\mathbb{1}-\sum_{j} \frac{1}{f_{j}(\sigma)} t_{0 j}^{(\rho)}(\sigma)\right)}\right)\left(\prod_{\rho<\kappa} z_{\rho \kappa}^{\frac{T_{a}^{(\kappa)} \eta^{a b} T_{b}^{(\rho)}}{k} \sum_{j} \frac{1}{f_{j}(\sigma)} t_{0 j}^{(\kappa)}(\sigma) t_{0 j}^{(\rho)}(\sigma)}\right) \\
& \times \prod_{\rho<\kappa} \exp \left\{\frac{T_{a}^{(\kappa)} \eta^{a b} T_{b}^{(\rho)}}{k} \sum_{j} \sum_{\hat{j}=1}^{f_{j}(\sigma)-1} \frac{1}{f_{j}(\sigma)} t_{\hat{j} j}^{(\kappa)}(\sigma) t_{-\hat{j}, j}^{(\rho)}(\sigma) I_{\frac{\hat{j}}{f_{j}(\sigma)}}\left(\frac{z_{\rho}}{z_{\kappa}}, \infty\right)\right\} \\
& =C_{+}(\mathcal{T}, \sigma) \prod_{j}\left\{\left(\prod_{\rho} z_{\rho}^{-\Delta\left(T^{(\rho)}\right)\left(1-\frac{1}{f_{j}(\sigma)}\right) t_{0 j}^{(\rho)}(\sigma)}\right)\left(\prod_{\rho<\kappa} z_{\rho \kappa}^{\frac{T_{a}^{(\kappa)} \eta^{a b} T_{b}^{(\rho)}}{k f_{j}(\sigma)} t_{0 j}^{(\kappa)}(\sigma) t_{0 j}^{(\rho)}(\sigma)}\right)\right. \\
& \left.\times \prod_{\rho<\kappa} \exp \left(\frac{T_{a}^{(\kappa)} \eta^{a b} T_{b}^{(\rho)}}{k f_{j}(\sigma)} \sum_{\hat{j}=1}^{f_{j}(\sigma)-1} t_{\hat{j} j}^{(\kappa)}(\sigma) t_{-\hat{j}, j}^{(\rho)}(\sigma) I_{\frac{\hat{j}}{f_{j}(\sigma)}}\left(\frac{z_{\rho}}{z_{\kappa}}, \infty\right)\right)\right\}
\end{aligned}
$$




$$
C_{+}(\mathcal{T}, \sigma)\left(\sum_{\rho=1}^{N} T_{a}^{(\rho)} t_{0 j}^{(\rho)}(\sigma)\right)=0, \quad a=1 \ldots \operatorname{dim}(\operatorname{Cartan} \mathfrak{g}), \quad \forall j, \quad \sigma=0, \ldots, N_{c}-1
$$

as a special case of (2.39). The $j$-factorized form (2.55b) of the correlators again reflects the semisimplicity of the abelian orbifold affine algebra (2.520).

\subsection{Subexample: Abelian cyclic permutation orbifolds}

We may be more explicit for the case $H$ (permutation) $=\mathbb{Z}_{\lambda}$ by substitution of the data

$$
\begin{gathered}
K=\lambda, \quad f_{j}(\sigma)=\rho(\sigma), \quad \hat{j}=\bar{r}=0, \ldots, \rho(\sigma)-1, \quad j=0, \ldots, \frac{\lambda}{\rho(\sigma)}-1 \\
a=1 \ldots \operatorname{dim}(\text { Cartan } \mathfrak{g}), \quad \sigma=0, \ldots, \lambda-1
\end{gathered}
$$

into the more general results above. Here $\rho(\sigma)$ is the order of $h_{\sigma} \in \mathbb{Z}_{\lambda}$, and we have also changed $\hat{j} \rightarrow r$ in accord with the prior convention [1, 2, 3, 11, 4, 10] for cyclic orbifolds. This gives the results for each sector $\sigma$ of the abelian cyclic permutation orbifolds $A_{\text {Cartan } g}\left(\mathbb{Z}_{\lambda}\right) / \mathbb{Z}_{\lambda}$ :

$$
\begin{aligned}
& \hat{J}_{r a j}\left(z e^{2 \pi i}\right)=e^{-2 \pi i \frac{r}{\rho(\sigma)}} \hat{J}_{r a j}(z), \quad \hat{J}_{r \pm \rho(\sigma), a j}(z)=\hat{J}_{r a j}(z) \\
& L_{\sigma}(m)=\frac{1}{2 k \rho(\sigma)} \sum_{j=0}^{\frac{\lambda}{\rho(\sigma)}} \sum_{r=0}^{-1} \sum_{p \in \mathbb{Z}} \eta^{a b}: \hat{J}_{r a j}\left(p+\frac{r}{\rho(\sigma)}\right) \hat{J}_{-r, b j}\left(m-p-\frac{r}{\rho(\sigma)}\right):_{M}+\delta_{m, 0} \hat{\Delta}_{0}(\sigma) \\
& \hat{c}(\sigma)=c=\lambda \operatorname{dim}(\operatorname{Cartan} \mathfrak{g}), \quad \hat{\Delta}_{0}(\sigma)=\frac{\lambda}{24} \operatorname{dim}(\operatorname{Cartan} \mathfrak{g})\left(1-\frac{1}{\rho^{2}(\sigma)}\right) \\
& \mathcal{T}_{\text {raj }}(T, \sigma)=T_{a} t_{r j}(\sigma), \quad\left[\mathcal{T}_{r a j}(T, \sigma), \mathcal{T}_{s b l}(T, \sigma)\right]=0 \\
& {\left[T_{a}, T_{b}\right]=0, \quad t_{r \pm \rho(\sigma), j}(\sigma)=t_{r j}(\sigma), \quad t_{r j}(\sigma)_{s l}{ }^{t m}=\delta_{j l} \delta_{l}^{m} \delta_{r+s-t, 0 \bmod \rho(\sigma)}} \\
& \hat{g}_{+}(\mathcal{T}, z, \sigma)=z^{-\Delta(T) \frac{\rho(\sigma)-1}{\rho(\sigma)}} \hat{\Gamma}\left(\mathcal{T}, \hat{J}_{0}(0), \sigma\right) e^{i T_{a} \sum_{j=0}^{\frac{\lambda}{\rho(\sigma)}-1} \hat{q}^{a j}(\sigma) t_{0 j}(\sigma)} z^{\frac{\eta^{a b} T_{b}}{\rho(\sigma) k} \sum_{j=0}^{\frac{\lambda}{\rho(\sigma)}-1}} \hat{J}_{0 a j}(0) t_{0 j}(\sigma) \\
& \times \exp \left(-\frac{\eta^{a b} T_{b}}{\rho(\sigma) k} \sum_{r=0}^{\rho(\sigma)-1} \sum_{j=0}^{\frac{\lambda}{\rho(\sigma)}-1} \sum_{m \leq-1} \hat{J}_{r a j}\left(m+\frac{r}{\rho(\sigma)}\right) \frac{z^{-\left(m+\frac{r}{\rho(\sigma)}\right)}}{m+\frac{r}{\rho(\sigma)}} t_{-r, j}(\sigma)\right) \\
& \times \exp \left(-\frac{\eta^{a b} T_{b}}{\rho(\sigma) k} \sum_{j=0}^{\frac{\lambda}{\rho(\sigma)}-1} \sum_{m \geq 1} \hat{J}_{0 a j}(m) \frac{z^{-m}}{m} t_{0 j}(\sigma)\right) \\
& \times \exp \left(-\frac{\eta^{a b} T_{b}}{\rho(\sigma) k} \sum_{r=1}^{\rho(\sigma)-1} \sum_{j=0}^{\frac{\lambda}{\rho(\sigma)}-1} \sum_{m \geq 0} \hat{J}_{r a j}\left(m+\frac{r}{\rho(\sigma)}\right) \frac{z^{-\left(m+\frac{r}{\rho(\sigma)}\right)}}{m+\frac{r}{\rho(\sigma)}} t_{-r, j}(\sigma)\right)
\end{aligned}
$$




$$
\begin{aligned}
& {\left[\hat{q}^{a j}(\sigma), \hat{J}_{r b l}\left(m+\frac{r}{\rho(\sigma)}\right)\right]=i \delta_{l}^{j} \delta_{b}^{a} \delta_{m+\frac{r}{\rho(\sigma)}, 0}, \quad\left[\hat{q}^{a j}(\sigma), \hat{q}^{b l}(\sigma)\right]=0} \\
& {\left[\hat{J}_{r a j}\left(m+\frac{r}{\rho(\sigma)}\right), \hat{J}_{s b l}\left(n+\frac{s}{\rho(\sigma)}\right)\right]=\delta_{j l} k \rho(\sigma) \eta_{a b}\left(m+\frac{r}{\rho(\sigma)}\right) \delta_{m+n+\frac{r+s}{\rho(\sigma)}, 0}} \\
& \hat{J}_{r \pm \rho(\sigma), a j}\left(m+\frac{r \pm \rho(\sigma)}{\rho(\sigma)}\right)=\hat{J}_{r a j}\left(m \pm 1+\frac{r}{\rho(\sigma)}\right) \\
& \left\langle\hat{g}_{+}\left(\mathcal{T}^{(1)}, z_{1}, \sigma\right) \cdots \hat{g}_{+}\left(\mathcal{T}^{(N)}, z_{N}, \sigma\right)\right\rangle_{\sigma} \\
& =C_{+}(\mathcal{T}, \sigma)\left(\prod_{\rho} z_{\rho}^{-\frac{\rho(\sigma)-1}{\rho(\sigma)} \Delta\left(T^{(\rho)}\right)}\right)\left(\prod_{\rho<\kappa} z_{\rho \kappa}^{\frac{T_{a}^{(\kappa)} \eta^{a b} T_{b}^{(\rho)}}{\rho(\sigma) k} \sum_{j=0}^{\frac{\lambda}{\rho(\sigma)}-1} t_{0 j}^{(\kappa)}(\sigma) t_{0 j}^{(\rho)}(\sigma)}\right) \\
& \times \prod_{\rho<\kappa} \exp \left\{\frac{T_{a}^{(\kappa)} \eta^{a b} T_{b}^{(\rho)}}{\rho(\sigma) k} \sum_{r=1}^{\rho(\sigma)-1} \sum_{j=0}^{\frac{\lambda}{\rho(\sigma)}-1} t_{r j}^{(\kappa)}(\sigma) t_{-r, j}^{(\rho)}(\sigma) I_{\frac{r}{\rho(\sigma)}}\left(\frac{z_{\rho}}{z_{\kappa}}, \infty\right)\right\} \\
& C_{+}(\mathcal{T}, \sigma)\left(\sum_{\rho=1}^{N} T_{a}^{(\rho)} t_{0 j}^{(\rho)}(\sigma)\right)=0, \quad a=1 \ldots \operatorname{dim}(\operatorname{Cartan} \mathfrak{g}), \quad j=0, \ldots \frac{\lambda}{\rho(\sigma)}-1 .
\end{aligned}
$$

The $j$-factorized forms (see Eqs. (2.53) and (2.55b) of these twisted vertex operators and correlators are also easily obtained.

When $\lambda=$ prime, these results simplify in the twisted sectors because

$$
\rho(\sigma)=\lambda=\text { prime }, \quad \bar{r}, \bar{s}=0, \ldots, \lambda-1, \quad j, l=0, \quad \sigma=1, \ldots, \lambda-1 .
$$

In this case it is conventional to suppress the degeneracy indices $j, l=0$, and we obtain:

$$
\begin{gathered}
\hat{J}_{r a} \equiv \hat{J}_{r a 0}, \quad \mathcal{T}_{r a 0} \equiv \mathcal{T}_{r a}=T_{a} t_{r}, \quad t_{r} \equiv t_{r}(\sigma) \\
\hat{J}_{r a}\left(z e^{2 \pi i}\right)=e^{-2 \pi i \frac{r}{\lambda}} \hat{J}_{r a}(z), \quad \hat{J}_{r \pm \lambda, a}(z)=\hat{J}_{r a}(z) \\
{\left[\hat{J}_{r a}\left(m+\frac{r}{\lambda}\right), \hat{J}_{s b}\left(n+\frac{s}{\lambda}\right)\right]=k \lambda \eta_{a b}\left(m+\frac{r}{\lambda}\right) \delta_{m+n+\frac{r+s}{\lambda}, 0}, \quad \hat{J}_{r \pm \lambda, a}\left(m+\frac{r \pm \lambda}{\lambda}\right)=\hat{J}_{r a}\left(m \pm 1+\frac{r}{\lambda}\right)} \\
L_{\sigma}(m)=\frac{1}{2 k \lambda} \sum_{r=0}^{\lambda-1} \sum_{p \in \mathbb{Z}} \eta^{a b}: \hat{J}_{r a}\left(p+\frac{r}{\lambda}\right) \hat{J}_{-r, b}\left(m-p-\frac{r}{\lambda}\right):_{M}+\delta_{m, 0} \hat{\Delta}_{0}(\sigma) \\
\hat{\Delta}_{0}(\sigma)=\frac{1}{24} \operatorname{dim}(\operatorname{Cartan} \mathfrak{g})\left(\lambda-\frac{1}{\lambda}\right)
\end{gathered}
$$




$$
\begin{gathered}
{\left[\hat{J}_{r a}\left(m+\frac{r}{\lambda}\right), \hat{g}_{+}(\mathcal{T}, z, \sigma)\right]=\hat{g}_{+}(\mathcal{T}, z, \sigma) T_{a} t_{r} z^{m+\frac{r}{\lambda}}} \\
t_{r} t_{s}=t_{r+s}, \quad t_{r \pm \lambda}=t_{r}, \quad\left(t_{r}\right)_{s}{ }^{t}=\delta_{r+s-t, 0 \bmod \lambda}, \quad t_{0}=\mathbb{1} . \\
a=1 \ldots \operatorname{dim}(\text { Cartan } \mathfrak{g}), \quad \bar{r}, \bar{s}=0, \ldots, \lambda-1, \quad \sigma=1, \ldots, \lambda-1 .
\end{gathered}
$$

Note that these relations are independent of $\sigma$. This extends a well-known fact [1] about $\lambda=$ prime cyclic permutation orbifolds - abelian or nonabelian - that their twisted current-algebraic formulation is independent of $\sigma$ in the twisted sectors.

Indeed, defining $\hat{q} \equiv \hat{q}(\sigma)$ and taking a $\sigma$-independent Klein transformation $\hat{\Gamma}$ we find the $\sigma$-independent twisted vertex operators and correlators

$$
\begin{aligned}
& \hat{g}_{+}(\mathcal{T}, z, \sigma)=z^{-\Delta(T) \frac{\lambda-1}{\lambda}} \hat{\Gamma}\left(\mathcal{T}, \hat{J}_{0}(0)\right) e^{i \hat{q}^{a} T_{a}} z^{\frac{\eta^{a b} T_{b}}{\lambda k} \hat{J}_{0 a}(0)} \\
& \times \exp \left(-\frac{\eta^{a b} T_{b}}{\lambda k} \sum_{r=0}^{\lambda-1} \sum_{m \leq-1} \hat{J}_{r a}\left(m+\frac{r}{\lambda}\right) \frac{z^{-\left(m+\frac{r}{\lambda}\right)}}{m+\frac{r}{\lambda}} t_{-r}\right) \\
& \times \exp \left(-\frac{\eta^{a b} T_{b}}{\lambda k} \sum_{m \geq 1} \hat{J}_{0 a}(m) \frac{z^{-m}}{m}\right) \\
& \times \exp \left(-\frac{\eta^{a b} T_{b}}{\lambda k} \sum_{r=1}^{\lambda-1} \sum_{m \geq 0} \hat{J}_{r a}\left(m+\frac{r}{\lambda}\right) \frac{z^{-\left(m+\frac{r}{\lambda}\right)}}{m+\frac{r}{\lambda}} t_{-r}\right) \\
& {\left[\hat{q}^{a}, \hat{J}_{r b}\left(m+\frac{r}{\lambda}\right)\right]=i \delta_{b}^{a} \delta_{m+\frac{r}{\lambda}, 0}, \quad\left[\hat{q}^{a}, \hat{q}^{b}\right]=0, \quad, \quad \sigma=1, \ldots, \lambda-1} \\
& \left\langle\hat{g}_{+}\left(\mathcal{T}^{(1)}, z_{1}, \sigma\right) \cdots \hat{g}_{+}\left(\mathcal{T}^{(N)}, z_{N}, \sigma\right)\right\rangle_{\sigma} \\
& =C_{+}(\mathcal{T})\left(\prod_{\rho} z_{\rho}^{-\frac{\lambda-1}{\lambda} \Delta\left(T^{(\rho)}\right)}\right)\left(\prod_{\rho<\kappa} z_{\rho \kappa}^{\frac{T_{a}^{(\kappa)} \eta^{a b} T_{b}^{(\rho)}}{\lambda k}}\right) \\
& \times \prod_{\rho<\kappa} \exp \left\{\frac{T_{a}^{(\kappa)} \eta^{a b} T_{b}^{(\rho)}}{\lambda k} \sum_{r=1}^{\lambda-1} t_{r}^{(\kappa)} t_{-r}^{(\rho)} I_{\frac{r}{\lambda}}\left(\frac{z_{\rho}}{z_{\kappa}}, \infty\right)\right\} \\
& C_{+}(\mathcal{T})\left(\sum_{\rho=1}^{N} T_{a}^{(\rho)}\right)=0, \quad a=1 \ldots \operatorname{dim}(\text { Cartan } \mathfrak{g}), \quad \sigma=1, \ldots, \lambda-1
\end{aligned}
$$

in each twisted sector of these orbifolds.

Finally for the special case $H$ (permutation) $=\mathbb{Z}_{2}$ we find for the single twisted sector $\sigma=1$

$$
\hat{J}_{0 a}\left(z e^{2 \pi i}\right)=\hat{J}_{0 a}(z), \quad \hat{J}_{1 a}\left(z e^{2 \pi i}\right)=-\hat{J}_{1 a}(z), \quad \hat{\Delta}_{0}=\frac{1}{16} \operatorname{dim}(\operatorname{Cartan} \mathfrak{g})
$$




$$
\begin{aligned}
&\left\langle\hat{g}_{+}\left(\mathcal{T}^{(1)}, z_{1}\right) \cdots \hat{g}_{+}\left(\mathcal{T}^{(N)}, z_{N}\right)\right\rangle= C_{+}(\mathcal{T})\left(\prod_{\rho} z_{\rho}^{-\frac{1}{2} \Delta\left(T^{(\rho)}\right)}\right)\left(\prod_{\rho<\kappa} z_{\rho \kappa}^{\left.\frac{1}{2 k} T_{a}^{(\kappa)} \eta^{a b} T_{b}^{(\rho)}\right)}\right. \\
& \times \prod_{\rho<\kappa}\left(\frac{\sqrt{z_{\rho}}-\sqrt{z_{\kappa}}}{\sqrt{z_{\rho}}+\sqrt{z_{\kappa}}}\right)^{\frac{T_{a}^{(\kappa)} \eta^{a b} T_{b}^{(\rho)}}{2 k} t_{1}^{(\kappa)} t_{1}^{(\rho)}} \\
& C_{+}(\mathcal{T})\left(\sum_{\rho=1}^{N} T_{a}^{(\rho)}\right)=0, \quad a=1 \ldots \operatorname{dim}(\operatorname{Cartan} \mathfrak{g}), \quad t_{1}^{(\kappa)}=t_{-1}^{(\kappa)}=\tau_{1}^{(\kappa)}
\end{aligned}
$$

where $\tau_{1}$ is the first Pauli matrix. Here we used the identities

$$
I_{\frac{1}{2}}(y, \infty)=\int_{\infty}^{y} \frac{d x}{x-1} x^{-\frac{1}{2}}=-\sum_{n=0}^{\infty} \frac{1}{n+\frac{1}{2}} y^{-\left(n+\frac{1}{2}\right)}, \quad e^{I_{\frac{1}{2}}(y, \infty)}=\frac{\sqrt{y}-1}{\sqrt{y}+1}
$$

given in Ref. [10].

\subsection{Example: The inversion orbifold $A_{\text {Cartan } \mathfrak{s u}(2)}\left(\mathbb{Z}_{2}\right) / \mathbb{Z}_{2}$}

The orbifold we consider here is a version of the outer-automorphic inversion orbifold

$$
\frac{\mathfrak{u}(1)}{\mathbb{Z}_{2}}: \quad J(z)^{\prime}=-J(z), \quad \sigma=1 .
$$

In particular, we study the realization of this orbifold obtained by embedding the $\mathfrak{u}(1)$ in an ambient $\mathfrak{s u}(2)$

$$
\frac{A_{\operatorname{Cartan} \mathfrak{s u}(2)}\left(\mathbb{Z}_{2}\right)}{\mathbb{Z}_{2}}: \quad J_{3}(z)^{\prime}=-J_{3}(z), \quad \sigma=1
$$

which is a simple example in our class of abelian orbifolds. Note in particular that this embedding promotes the outer automorphism $J(z)^{\prime}=-J(z)$ of the abelian current algebra into an inner automorphism of the ambient $\mathfrak{s u}(2)$ current algebra.

In this case the $c=1$ stress tensor and untwisted currents of the symmetric CFT are

$$
T(z)=\frac{1}{2 k}: J_{3}(z) J_{3}(z):, \quad J_{3}(z) J_{3}(w)=\frac{k}{(z-w)^{2}}+\mathcal{O}(z-w)^{0}
$$

where $k$ is the level of the ambient affine $\mathfrak{s u}(2)$. The solution to the $H$-eigenvalue problem in the twisted sector is very simple

$$
\omega U^{\dagger}=U^{\dagger} E \quad: \quad E=\omega=-1, \quad \rho=2, \quad \bar{n}(r)=\overline{-n(r)}=1, \quad U^{\dagger}=1
$$

and, choosing the normalization $\chi=1$, we obtain the simple duality transformations

$$
\mathcal{G}_{1,-1}=k, \quad \mathcal{G}^{1,-1}=\frac{1}{k}, \quad \mathcal{L}_{1,-1}=\frac{1}{2 k} .
$$


Then the twisted sector of the orbifold is described by the $\hat{c}=1$ stress tensor and twisted currents

$$
\begin{gathered}
\hat{T}(z)=\frac{1}{2 k}: \hat{J}_{1}(z) \hat{J}_{-1}(z):, \quad \hat{J}_{1}\left(z e^{2 \pi i}\right)=-\hat{J}_{1}(z), \quad \hat{J}_{1 \pm 2}(z)=\hat{J}_{1}(z) \\
\hat{J}_{1}(z)=\sum_{m \in \mathbb{Z}} \hat{J}_{1}\left(m+\frac{1}{2}\right) z^{-\left(m+\frac{1}{2}\right)-1}, \quad \hat{J}_{1 \pm 2}\left(m+\frac{1 \pm 2}{2}\right)=\hat{J}_{1}\left(m \pm 1+\frac{1}{2}\right) \\
L(m)=\frac{1}{2 k} \sum_{p \in \mathbb{Z}}: \hat{J}_{1}\left(p+\frac{1}{2}\right) \hat{J}_{-1}\left(m-p-\frac{1}{2}\right):_{M}+\delta_{m, 0} \frac{1}{16} \\
{\left[\hat{J}_{1}\left(m+\frac{1}{2}\right), \hat{J}_{1}\left(n+\frac{1}{2}\right)\right]=k\left(m+\frac{1}{2}\right) \delta_{m+n+1,0}, \quad \hat{J}_{1}\left(m+\frac{1}{2}\right)^{\dagger}=\hat{J}_{-1}\left(-m-\frac{1}{2}\right)=\hat{J}_{1}\left(-m-1+\frac{1}{2}\right)} \\
\hat{J}_{1}\left(\left(m+\frac{1}{2}\right) \geq 0\right)|0\rangle=\left\langle 0\left|\hat{J}_{1}\left(\left(m+\frac{1}{2}\right) \leq 0\right)=0, \quad\left(L(m \geq 0)-\delta_{m, 0} \frac{1}{16}\right)\right| 0\right\rangle=0
\end{gathered}
$$

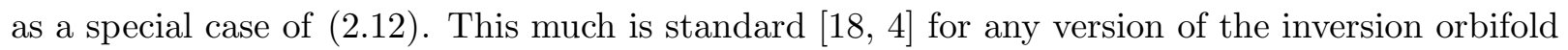
$\mathfrak{u}(1) / \mathbb{Z}_{2}$.

In current-algebraic orbifold theory, the representation theory of the orbifold begins by finding the action $W\left(h_{\sigma} ; T\right)$ of $h_{\sigma} \in H$ in representation $T$, which solves the linkage relation [10]

$$
W^{\dagger}\left(h_{\sigma} ; T\right) T_{a} W\left(h_{\sigma} ; T\right)=w\left(h_{\sigma}\right)_{a}^{b} T_{b}, \quad \omega\left(h_{\sigma}\right), W\left(h_{\sigma} ; T\right) \in H
$$

given the action $\omega\left(h_{\sigma}\right)$ of $h_{\sigma} \in H$ in the adjoint (see Eq. (2.4)). For our realization $A_{\text {Cartan } \mathfrak{s u}(2)}\left(\mathbb{Z}_{2}\right) / \mathbb{Z}_{2}$ of the inversion orbifold, we introduce the weight basis of the ambient $\mathfrak{s u}(2)$

$$
\begin{gathered}
{\left[T_{A}, T_{B}\right]=i \epsilon_{A B C} T_{C}, \quad, \quad A=1,2,3, \quad \epsilon_{123}=1} \\
\left(T_{A}\right)_{m^{\prime}}{ }^{m}=\left\langle j m^{\prime}\left|J_{A}(0)\right| j m\right\rangle, \quad\left(T_{3}\right)_{m^{\prime}}{ }^{m}=m \delta_{m^{\prime}}^{m}, \quad\left|m^{\prime}\right|,|m| \leq j, \quad j=0, \frac{1}{2}, 1, \ldots
\end{gathered}
$$

where we have chosen root length $\alpha^{2}=1$. Then the linkage relation and its solution for any spin $j$ are

$$
\begin{gathered}
W^{\dagger}(T) T_{3} W(T)=-T_{3}, \quad W^{\dagger}(T) W(T)=\mathbb{1} \\
W(T)=e^{i \pi T_{2}}, \quad R(T)=R(j)= \begin{cases}2 & \text { for integer } j \\
4 & \text { for half integer } j\end{cases}
\end{gathered}
$$

where $R(T)$ is the order of $W(T)$. Note that $W(T) \in S U(2)$, which reflects our promotion of the outer automorphism (2.66) to an inner automorphism of the ambient $\mathfrak{s u}(2)$. The affine cutoff is $j \leq x / 2, x=2 k$ in this case. 
The next step is to solve the extended $H$-eigenvalue problem 2.15) for representation $T$. In this case, the extended $H$-eigenvalue problem and its solution are

$$
\begin{gathered}
\sum_{n^{\prime}=-j}^{j} W(T)_{n}{ }^{n^{\prime}} U^{\dagger}(T)_{n^{\prime}}{ }^{m}=U^{\dagger}(T)_{n}{ }^{m} E_{m}(T), \quad|n|,|m| \leq j \\
U^{\dagger}(T)=e^{-i \frac{\pi}{2} T_{1}}, \quad E_{m}(T)=e^{i \pi m}=e^{-2 \pi i \frac{N(r)}{R(T)}}, \quad N(r)=-2 m R(T)
\end{gathered}
$$

where $U^{\dagger}(T)$ is again in $S U(2)$. Then we may use (2.14e) to obtain the twisted representation matrices $\mathcal{T}$ in the twisted sector

$$
\begin{gathered}
\hat{J}_{1}(z) \hat{g}_{+}(\mathcal{T}, w)=\frac{\hat{g}_{+}(\mathcal{T}, w)}{z-w} \mathcal{T}(T)+\mathcal{O}(z-w)^{0} \\
\mathcal{T}(T) \equiv \mathcal{T}_{ \pm 1}(T)=U(T) T_{3} U^{\dagger}(T)=-T_{2}, \quad E(T)_{m^{\prime}}{ }^{m}=\delta_{m^{\prime}}^{m} e^{i \pi m} \\
\mathcal{T}(T)=-E(T) \mathcal{T}(T) E^{*}(T) \quad \leftrightarrow \quad\left(T_{2}\right)_{m^{\prime}}{ }^{m}\left(1+e^{i \pi\left(m^{\prime}-m\right)}\right)=0
\end{gathered}
$$

where the $\mathcal{T}$-selection rule in (2.76c) is self-evident because $\left(T_{2}\right)_{m^{\prime}}{ }^{m}$ satisfies $|\Delta m|=1$.

Using this data, the twisted vertex operator equation and its solution, the twisted vertex operators

$$
\begin{aligned}
& \partial \hat{g}_{+}(\mathcal{T}, z)=\frac{\mathcal{T}}{k}: \hat{J}_{1}(z) \hat{g}_{+}(\mathcal{T}, z):_{M}-\frac{\mathcal{T}^{2}}{2 k z} \hat{g}_{+}(\mathcal{T}, z), \quad \mathcal{T} \equiv \mathcal{T}(T)=-T_{2} \\
& \hat{g}_{+}(\mathcal{T}, z)=z^{-\frac{\mathcal{T}^{2}}{2 k}} \exp \left(-\frac{\mathcal{T}}{k} \sum_{m \leq-1} \hat{J}_{1}\left(m+\frac{1}{2}\right) \frac{z^{-\left(m+\frac{1}{2}\right)}}{m+\frac{1}{2}}\right) \exp \left(-\frac{\mathcal{T}}{k} \sum_{m \geq 0} \hat{J}_{1}\left(m+\frac{1}{2}\right) \frac{z^{-\left(m+\frac{1}{2}\right)}}{m+\frac{1}{2}}\right) \\
& {\left[\hat{J}_{1}\left(m+\frac{1}{2}\right), \hat{g}_{+}(\mathcal{T}, z)\right]=\hat{g}_{+}(\mathcal{T}, z) \mathcal{T} z^{m+\frac{1}{2}}, \quad\left[L(m), \hat{g}_{+}(\mathcal{T}, z)\right]=z^{m}\left(z \partial_{z}+\frac{\mathcal{T}^{2}}{2 k}(m+1)\right) \hat{g}_{+}(\mathcal{T}, z)} \\
& \hat{g}_{+}\left(\mathcal{T}^{(1)}, z_{1}\right) \hat{g}_{+}\left(\mathcal{T}^{(2)}, z_{2}\right)=: \hat{g}_{+}\left(\mathcal{T}^{(1)}, z_{1}\right) \hat{g}_{+}\left(\mathcal{T}^{(2)}, z_{2}\right):_{M}\left(\frac{\sqrt{z_{1}}-\sqrt{z_{2}}}{\sqrt{z_{1}}+\sqrt{z_{2}}}\right)^{\frac{\mathcal{T}^{(2)} \mathcal{T}^{(1)}}{k}}
\end{aligned}
$$

can be read off (ignore all structure with zero twist class $\bar{n}(r)=0$ ) from Eq. 2.16a), the general results of Subsecs. 2.3, 2.4 and the identity (2.65).

Similarly, the correlators of the twisted vertex operators

$$
\left\langle\hat{g}_{+}\left(\mathcal{T}^{(1)}, z_{1}\right) \cdots \hat{g}_{+}\left(\mathcal{T}^{(N)}, z_{N}\right)\right\rangle=\left(\prod_{\rho} z_{\rho}^{-\frac{1}{2 k} \mathcal{T}^{(\rho)} \mathcal{T}^{(\rho)}}\right) \prod_{\rho<\kappa}\left(\frac{\sqrt{z_{\rho}}-\sqrt{z_{\kappa}}}{\sqrt{z_{\rho}}+\sqrt{z_{\kappa}}}\right)^{\frac{\mathcal{T}^{(\kappa)} \mathcal{T}^{(\rho)}}{k}}
$$

are easily read from the data and Eq. (2.39). The absence of a global Ward identity is another consequence of the absence of zero twist class in the inversion orbifold. 


\subsection{The twisted right-mover sectors of $A_{\text {Cartan } g}(H) / H$}

We turn now to the twisted right-mover sectors of our class $A_{\text {Cartan } g}(H) / H$ of abelian orbifolds, whose description may be read off as the abelian limit of the right-mover sectors of the general WZW orbifold in Ref. [10].

One finds in Ref. [10] that the twisted right-mover currents

$$
\begin{aligned}
& \hat{\bar{J}}_{n(r) \mu}\left(\bar{z} e^{-2 \pi i}, \sigma\right)=e^{-2 \pi i \frac{n(r)}{\rho(\sigma)}} \hat{\bar{J}}_{n(r) \mu}(\bar{z}, \sigma), \quad \hat{\bar{J}}_{n(r) \mu}(\bar{z}, \sigma)=\sum_{m \in \mathbb{Z}} \hat{\bar{J}}_{n(r) \mu}\left(m+\frac{n(r)}{\rho(\sigma)}\right) \bar{z}^{\left(m+\frac{n(r)}{\rho(\sigma)}\right)-1} \\
& \hat{\bar{J}}_{n(r) \pm \rho(\sigma), \mu}(\bar{z}, \sigma)=\hat{\bar{J}}_{n(r) \mu}(\bar{z}, \sigma), \quad \hat{\bar{J}}_{n(r) \pm \rho(\sigma), \mu}\left(m+\frac{n(r) \pm \rho(\sigma)}{\rho(\sigma)}\right)=\hat{\bar{J}}_{n(r) \mu}\left(m \pm 1+\frac{n(r)}{\rho(\sigma)}\right)
\end{aligned}
$$

have the same monodromies as the twisted left-mover currents when the same path is followed. Moreover, the twisted right-mover Virasoro operators, twisted right-mover current algebra and ground state conformal weights are easily read from Ref. 10]

$$
\begin{gathered}
\bar{L}_{\sigma}(m)=\frac{1}{2} \mathcal{G}^{n(r) \mu ;-n(r), \nu}(\sigma)\left\{\sum_{p \in \mathbb{Z}}: \hat{\bar{J}}_{n(r) \mu}\left(p+\frac{n(r)}{\rho(\sigma)}\right) \hat{\bar{J}}_{-n(r), \nu}\left(-m-p-\frac{n(r)}{\rho(\sigma)}\right):_{\bar{M}}\right. \\
\left.+\delta_{m, 0} \frac{\overline{-n(r)}}{2 \rho(\sigma)}\left(1-\frac{\overline{-n(r)}}{\rho(\sigma)}\right) \mathcal{G}_{n(r) \mu ;-n(r), \nu}(\sigma)\right\} \\
{\left[\hat{\bar{J}}_{n(r) \mu}\left(m+\frac{n(r)}{\rho(\sigma)}\right), \hat{\bar{J}}_{n(s) \nu}\left(n+\frac{n(s)}{\rho(\sigma)}\right)\right]=-\left(m+\frac{n(r)}{\rho(\sigma)}\right) \delta_{m+n+\frac{n(r)+n(s)}{\rho(\sigma)}, 0} \mathcal{G}_{n(r) \mu ;-n(r), \nu}(\sigma)} \\
\hat{\bar{J}}_{n(r) \mu}\left(m+\frac{n(r)}{\rho(\sigma)} \leq 0\right)|0\rangle_{\sigma}={ }_{\sigma}\langle 0| \hat{\bar{J}}_{n(r) \mu}\left(m+\frac{n(r)}{\rho(\sigma)} \geq 0\right)=0 \\
\left(\bar{L}_{\sigma}(m \geq 0)-\delta_{m, 0} \hat{\bar{\Delta}}_{0}(\sigma)|0\rangle_{\sigma}=0, \quad \hat{\bar{\Delta}}_{0}(\sigma)=\hat{\Delta}_{0}(\sigma)\right.
\end{gathered}
$$

where $\bar{M}$ normal ordering is defined in that reference and $\overline{-n(r)}$ and $\hat{\Delta}_{0}(\sigma)$ are given respectively in (2.32c) and (2.130). The central charge is $\overline{\hat{c}}(\sigma)=\hat{c}(\sigma)=c$. Similarly, the setup for the twisted right-mover vertex operators $\hat{g}_{-}(\mathcal{T}, \bar{z}, \sigma)$

$$
\begin{gathered}
{\left[\hat{\bar{J}}_{n(r) \mu}\left(m+\frac{n(r)}{\rho(\sigma)}\right), \hat{g}_{-}(\mathcal{T}, \bar{z}, \sigma)\right]=-\bar{z}^{-\left(m+\frac{n(r)}{\rho(\sigma)}\right)} \mathcal{T}_{n(r) \mu} \hat{g}_{-}(\mathcal{T}, \bar{z}, \sigma)} \\
{\left[L_{\sigma}(m), \hat{g}_{-}(\mathcal{T}, \bar{z}, \sigma)\right]=\bar{z}^{m}\left(\bar{z} \partial_{\bar{z}}+\mathcal{D}(\mathcal{T}, \sigma)(m+1)\right) \hat{g}_{-}(\mathcal{T}, \bar{z}, \sigma)} \\
\bar{\partial} \hat{g}_{-}(\mathcal{T}, \bar{z}, \sigma)=-\mathcal{G}^{n(r) \mu ;-n(r), \nu}(\sigma) \mathcal{T}_{-n(r), \nu}\left(: \hat{\bar{J}}_{n(r) \mu}(z) \hat{g}_{-}(\mathcal{T}, \bar{z}, \sigma):_{\bar{M}}+\frac{\overline{-n(r)}}{\rho(\sigma)} \frac{1}{\bar{z}} \mathcal{T}_{n(r) \mu} \hat{g}_{-}(\mathcal{T}, \bar{z}, \sigma)\right) \\
: \hat{\bar{J}}_{n(r) \mu}(\bar{z}) \hat{g}_{-}(\mathcal{T}, \bar{z}, \sigma):_{\bar{M}}=\hat{\bar{J}}_{n(r) \mu}^{+}(\bar{z}) \hat{g}_{-}(\mathcal{T}, \bar{z}, \sigma)+\hat{g}_{-}(\mathcal{T}, \bar{z}, \sigma) \hat{\bar{J}}_{n(r) \mu}^{-}(\bar{z})
\end{gathered}
$$




$$
\begin{aligned}
& \hat{\bar{J}}_{n(r) \mu}^{+}(\bar{z}) \equiv \sum_{m>0} \hat{\bar{J}}_{n(r) \mu}\left(m-\frac{\overline{-n(r)}}{\rho(\sigma)}\right) \bar{z}^{\left(m-\frac{\overline{-n(r)}}{\rho(\sigma)}\right)-1} \\
& \hat{\bar{J}}_{n(r) \mu}^{-}(\bar{z}) \equiv \sum_{m \leq 0} \hat{\bar{J}}_{n(r) \mu}\left(m-\frac{\overline{-n(r)}}{\rho(\sigma)}\right) \bar{z}^{\left(m-\frac{\overline{-n(r)}}{\rho(\sigma)}\right)-1}
\end{aligned}
$$

is easily read from Ref. [10]. The twisted representation matrices $\mathcal{T}(T, \sigma)$ and the twisted conformal weight matrix $\mathcal{D}(\mathcal{T}, \sigma)$ are given in (2.14). Again, we assume that the twisted right-mover vertex operators $\hat{g}_{-}(\mathcal{T}, \bar{z}, \sigma)$ are square matrices which commute with the twisted representation matrices $\mathcal{T}=\mathcal{T}(T, \sigma)$.

Note the minus sign on the right side of the twisted right-mover current algebra (2.80b). This sign change (relative to the twisted left-mover current algebra (2.7d)) is a universal phenomenon [10] in the right-mover sectors of all current-algebraic orbifolds. It is known for permutation orbifolds (and we will check below for the inversion orbifold) that the twisted right-mover current algebra is rectifiable [10] to a copy of the twisted left-mover current algebra (see also Subsec [3.5). More generally, it is known [10] for all current-algebraic orbifolds that the twisted right-mover current algebra of sector $\sigma \leftrightarrow h_{\sigma}$ is isomorphic to the twisted left-mover current algebra of sector $h_{\sigma}^{-1}$. Here we exploit this isomorphism to solve the twisted right-mover vertex operator equation (2.810).

Following Ref. [10], we first define the mode-number reversed right-mover currents

$$
\begin{gathered}
\hat{\bar{J}}_{n(r) \mu}^{\mathrm{R}}\left(m+\frac{n(r)}{\rho(\sigma)}\right) \equiv \hat{\bar{J}}_{-n(r), \mu}\left(-m-\frac{n(r)}{\rho(\sigma)}\right) \\
{\left[\hat{\bar{J}}_{n(r) \mu}^{\mathrm{R}}\left(m+\frac{n(r)}{\rho(\sigma)}\right), \hat{\bar{J}}_{n(s) \nu}^{\mathrm{R}}\left(n+\frac{n(s)}{\rho(\sigma)}\right)\right]=\left(m+\frac{n(r)}{\rho(\sigma)}\right) \delta_{m+n+\frac{n(r)+n(s)}{\rho(\sigma)}, 0} \tilde{\mathcal{G}}_{n(r), \mu ;-n(r), \nu}(\sigma) .}
\end{gathered}
$$

Here $\tilde{\mathcal{G}}(\sigma)$ is the twisted metric of sector $h_{\sigma}^{-1}$, which satisfies

$$
\tilde{\mathcal{G}}_{n(r) \mu ; n(s) \nu}(\sigma) \equiv \mathcal{G}_{-n(r), \mu ;-n(s), \nu}(\sigma) \propto \delta_{n(r)+n(s), 0 \bmod \rho(\sigma)}, \quad \tilde{\mathcal{G}}_{n(r) \mu ;-n(r), \nu}(\sigma)=\mathcal{G}_{n(r) \nu ;-n(r), \mu}(\sigma) .
$$

We will also need the inverse of $\tilde{\mathcal{G}}(\sigma)$ which satisfies

$$
\begin{gathered}
\tilde{\mathcal{G}}_{n(r) \mu ; n(t) \delta}(\sigma) \tilde{\mathcal{G}}^{n(t) \delta ; n(s) \nu}(\sigma)=\delta_{n(r) \mu}{ }^{n(s) \nu}, \quad \tilde{\mathcal{G}}^{n(r) \mu ;-n(r), \nu}(\sigma)=\mathcal{G}^{n(r) \nu ;-n(r), \mu}(\sigma) \\
\sum_{\delta} \tilde{\mathcal{G}}_{n(r) \mu ;-n(r) \delta}(\sigma) \tilde{\mathcal{G}}^{-n(r) \delta ; n(r) \nu}(\sigma)=\delta_{\mu}{ }^{\nu} .
\end{gathered}
$$

When the twisted right-mover current algebra is rectifiable one finds that $\tilde{\mathcal{G}}(\sigma)=\mathcal{G}(\sigma)$, but we will not need this fact here

We can also extend this isomorphism to the twisted representation matrices:

$$
\left[\hat{\bar{J}}_{n(r) \mu}^{\mathrm{R}}\left(m+\frac{n(r)}{\rho(\sigma)}\right), \hat{g}_{-}(\mathcal{T}, \bar{z}, \sigma)\right]=\bar{z}^{m+\frac{n(r)}{\rho(\sigma)}} \tilde{\mathcal{T}}_{n(r) \mu} \hat{g}_{-}(\mathcal{T}, \bar{z}, \sigma), \quad \tilde{\mathcal{T}}_{n(r) \mu}(\sigma) \equiv-\mathcal{T}_{-n(r), \mu}(\sigma)
$$


by rewriting Eq. 2.81a) in terms of $\hat{\bar{J}}^{\mathrm{R}}$ and the matrices $\tilde{\mathcal{T}}$. This relation is isomorphic to the analogous left-mover result (2.24a) because $\tilde{\mathcal{T}}$ commutes with $\hat{g}_{-}$.

Finally, the twisted right-mover vertex operator equation takes the form

$$
\begin{gathered}
\bar{\partial} \hat{g}_{-}(\mathcal{T}, \bar{z}, \sigma)=\tilde{\mathcal{G}}^{n(r) \mu ;-n(r), \nu}(\sigma) \tilde{\mathcal{T}}_{-n(r), \nu}\left(: \hat{\bar{J}}_{n(r) \mu}^{\mathrm{R}}(z) \hat{g}_{-}(\mathcal{T}, \bar{z}, \sigma):_{M}-\frac{\bar{n}(r)}{\rho(\sigma)} \frac{1}{\bar{z}} \tilde{\mathcal{T}}_{n(r) \mu} \hat{g}_{-}(\mathcal{T}, \bar{z}, \sigma)\right) \\
: \hat{\bar{J}}_{n(r) \mu}^{\mathrm{R}}(\bar{z}) \hat{g}_{-}(\mathcal{T}, \bar{z}, \sigma):_{M}=\hat{\bar{J}}_{n(r) \mu}^{\mathrm{R}-}(\bar{z}) \hat{g}_{-}(\mathcal{T}, \bar{z}, \sigma)+\hat{g}_{-}(\mathcal{T}, \bar{z}, \sigma) \hat{\bar{J}}_{n(r) \mu}^{\mathrm{R}+}(\bar{z}) \\
\hat{\bar{J}}_{n(r) \mu}^{\mathrm{R}-}(\bar{z}) \equiv \hat{\bar{J}}_{n(r) \mu}^{+}(\bar{z})=\sum_{m \leq-1} \hat{\bar{J}}_{\bar{n}(r) \mu}^{\mathrm{R}}\left(m+\frac{\bar{n}(r)}{\rho(\sigma)}\right) \bar{z}^{-\left(m+\frac{\bar{n}(r)}{\rho(\sigma)}\right)-1} \\
\hat{\bar{J}}_{n(r) \mu}^{\mathrm{R}+}(\bar{z}) \equiv \hat{\bar{J}}_{n(r) \mu}^{-}(\bar{z})=\sum_{m \geq 0} \hat{\bar{J}}_{\bar{n}(r) \mu}^{\mathrm{R}}\left(m+\frac{\bar{n}(r)}{\rho(\sigma)}\right) \bar{z}^{-\left(m+\frac{\bar{n}(r)}{\rho(\sigma)}\right)-1}
\end{gathered}
$$

where we have changed variable $n(r) \rightarrow-n(r)$ to obtain (2.86a) from (2.81c).

This completes the isomorphism with Eq. (2.16) and allows us to read off the twisted rightmover vertex operators of $A_{\text {Cartan } g}(H) / H$ on inspection from the left-mover results

$$
\begin{aligned}
& z \rightarrow \bar{z}, \quad \mathcal{T} \rightarrow \tilde{\mathcal{T}}, \quad \hat{J} \rightarrow \hat{\bar{J}}^{\mathrm{R}}, \quad \hat{\Gamma} \rightarrow \hat{\bar{\Gamma}} \\
& \hat{g}_{-}(\mathcal{T}, \bar{z}, \sigma)=\bar{z}^{-\tilde{\mathcal{G}}^{n(r) \mu ;-n(r), \nu}(\sigma) \frac{\bar{n}(r)}{\rho(\sigma)} \tilde{\mathcal{T}}_{n(r) \mu} \tilde{\mathcal{T}}_{-n(r), \nu}} \hat{\bar{\Gamma}}\left(\tilde{\mathcal{T}}, \hat{\bar{J}}_{0}^{\mathrm{R}}(0), \sigma\right) e^{i \hat{\bar{q}}^{\mu}(\sigma) \tilde{\mathcal{T}}_{0 \mu}}
\end{aligned}
$$

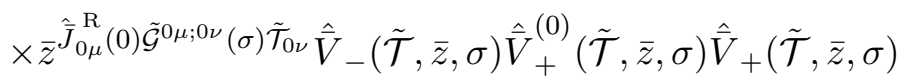

$$
\begin{aligned}
& \hat{\bar{V}}_{-}(\tilde{\mathcal{T}}, \bar{z}, \sigma) \equiv \exp \left\{-\tilde{\mathcal{G}}^{n(r) \mu ;-n(r), \nu}(\sigma) \sum_{m \leq-1} \hat{\bar{J}}_{\bar{n}(r) \mu}^{\mathrm{R}}\left(m+\frac{\bar{n}(r)}{\rho(\sigma)}\right) \frac{\bar{z}^{-\left(m+\frac{\bar{n}(r)}{\rho(\sigma)}\right)}}{m+\frac{\bar{n}(r)}{\rho(\sigma)}} \tilde{\mathcal{T}}_{-n(r), \nu}\right\} \\
& \hat{\bar{V}}_{+}^{(0)}(\tilde{\mathcal{T}}, \bar{z}, \sigma) \equiv \exp \left\{-\tilde{\mathcal{G}}^{0 \mu ; 0 \nu}(\sigma) \sum_{m \geq 1} \hat{\bar{J}}_{0 \mu}^{\mathrm{R}}(m) \frac{\bar{z}^{-m}}{m} \tilde{\mathcal{T}}_{0 \nu}\right\} \\
& \hat{\bar{V}}_{+}(\tilde{\mathcal{T}}, \bar{z}, \sigma) \equiv \exp \left\{-\tilde{\mathcal{G}}^{n(r) \mu ;-n(r), \nu}(\sigma)\left(1-\delta_{\bar{n}(r), 0}\right) \sum_{m \geq 0} \hat{\bar{J}}_{\bar{n}(r) \mu}^{\mathrm{R}}\left(m+\frac{\bar{n}(r)}{\rho(\sigma)}\right) \frac{\bar{z}^{-\left(m+\frac{\bar{n}(r)}{\rho(\sigma)}\right)}}{m+\frac{\bar{n}(r)}{\rho(\sigma)}} \tilde{\mathcal{T}}_{-n(r), \nu}\right\} \\
& {\left[\hat{\bar{q}}^{\mu}(\sigma), \hat{\bar{J}}_{n(s) \nu}^{\mathrm{R}}\left(n+\frac{n(s)}{\rho(\sigma)}\right)\right]=i \delta_{\nu}^{\mu} \delta_{n+\frac{n(s)}{\rho(\sigma)}, 0}, \quad\left[\hat{\bar{q}}^{\mu}(\sigma), \hat{\bar{q}}^{\nu}(\sigma)\right]=0, \quad \sigma=0, \ldots, N_{c}-1}
\end{aligned}
$$


where $\hat{\bar{\Gamma}}$ is an undetermined right-mover Klein transformation. The twisted mode algebra of $\hat{\bar{J}}^{\mathrm{R}}$ and the quantities $\tilde{\mathcal{G}}, \tilde{\mathcal{T}}$ are given in Eqs. (2.82), (2.83) and (2.85). At this point we verify our initial assumption that $\hat{g}_{-}$is a square matrix which commutes with the twisted representation matrices $\mathcal{T}$.

Using (2.87) we may now discuss for the twisted right-mover vertex operators all the properties given for the twisted left-mover vertex operators in Subsec. 2.4. For brevity we confine ourselves here to the following three topics.

\section{- Intrinsic monodromy of the right-mover vertex operators}

The monodromies of the twisted right-mover vertex operators follow the same line as given above for the left movers. The result in sector $\sigma$ of $A_{\text {Cartan } g}(H) / H$ is

$$
\begin{aligned}
\hat{g}_{-}\left(\mathcal{T}, \bar{z} e^{-2 \pi i}, \sigma\right)= & E(T, \sigma) \hat{g}_{-}(\mathcal{T}, \bar{z}, \sigma) E(T, \sigma)^{*} \\
& \times e^{2 \pi i\left(\tilde{\mathcal{G}}^{n(r) \mu ;-n(r), \nu}(\sigma) \frac{\bar{n}(r)}{\rho(\sigma)} \tilde{\mathcal{T}}_{n(r) \mu} \tilde{\mathcal{T}}_{-n(r), \nu}-\hat{\bar{J}}_{0 \mu}^{\mathrm{R}}(0) \tilde{\mathcal{G}}^{0 \mu ; 0 \nu}(\sigma) \tilde{\mathcal{T}}_{0 \nu}\right)} \\
e^{2 \pi i \frac{n(r)}{\rho(\sigma)}} \tilde{\mathcal{T}}_{-n(r), \nu}= & E(T, \sigma) \tilde{\mathcal{T}}_{-n(r), \nu} E(T, \sigma)^{*}, \quad \sigma=0, \ldots, N_{c}-1
\end{aligned}
$$

where the $\mathcal{T}$-selection rule (2.34a was used in the form (2.88b).

\section{- Right-mover orbifold correlators}

The right-mover correlators of sector $\sigma$ of $A_{\text {Cartan } g}(H) / H$ are:

$$
\begin{aligned}
& \hat{A}_{-}(\mathcal{T}, \bar{z}, \sigma) \quad \equiv\left\langle\hat{g}_{-}\left(\mathcal{T}^{(1)}, \bar{z}_{1}, \sigma\right) \cdots \hat{g}_{-}\left(\mathcal{T}^{(N)}, \bar{z}_{N}, \sigma\right)\right\rangle_{\sigma}, \quad \sigma=0, \ldots, N_{c}-1 \\
& =C_{-}(\tilde{\mathcal{T}}, \sigma)\left(\prod_{\rho} \bar{z}_{\rho}^{\left.-\tilde{\mathcal{G}}^{n(r) \mu ;-n(r), \nu}(\sigma) \frac{\bar{n}(r)}{\rho(\sigma)} \tilde{\mathcal{T}}_{n(r) \mu}^{(\rho)} \tilde{\mathcal{T}}_{-n(r), \nu}^{(\rho)}\right)\left(\prod_{\rho<\kappa} \tilde{z}_{\rho \kappa}^{(\kappa)} \tilde{\mathcal{G}}^{0 \mu ; 0 \nu}(\sigma) \tilde{\mathcal{T}}_{0 \nu}^{(\rho)}\right)}\right) \\
& \times \prod_{\rho<\kappa} \exp \left\{\tilde{\mathcal{T}}_{n(r) \mu}^{(\kappa)} \tilde{\mathcal{G}}^{n(r) \mu ;-n(r), \nu}(\sigma) \tilde{\mathcal{T}}_{-n(r), \nu}^{(\rho)}\left(1-\delta_{\bar{n}(r), 0}\right) I_{\frac{\bar{n}(r)}{\rho(\sigma)}}\left(\frac{\bar{z}_{\rho}}{\bar{z}_{\kappa}}, \infty\right)\right\} \\
& C_{-}(\tilde{\mathcal{T}}, \sigma) \equiv\left\langle\hat{G}_{-}\left(\tilde{\mathcal{T}}^{(1)}, \sigma\right) \cdots \hat{G}_{-}\left(\tilde{\mathcal{T}}^{(N)}, \sigma\right)\right\rangle_{\sigma}, \quad \hat{G}_{-}(\tilde{\mathcal{T}}, \sigma) \equiv \hat{\bar{\Gamma}}\left(\tilde{\mathcal{T}}, \hat{\bar{J}}_{0}^{\mathrm{R}}(0), \sigma\right) e^{i \hat{\bar{q}}^{\mu}(\sigma) \tilde{\mathcal{T}}_{0 \mu}} \\
& \left\langle\left[\hat{\bar{J}}_{0 \mu}^{\mathrm{R}}(0), \hat{G}_{-}\left(\tilde{\mathcal{T}}^{(1)}, \sigma\right) \cdots \hat{G}_{-}\left(\tilde{\mathcal{T}}^{(N)}, \sigma\right)\right]\right\rangle_{\sigma}=0 \quad \Rightarrow \quad\left(\sum_{\rho=1}^{N} \tilde{\mathcal{T}}_{0 \mu}^{(\rho)}\right) C_{-}(\tilde{\mathcal{T}}, \sigma)=0, \quad \forall \mu
\end{aligned}
$$

As for the twisted left movers, the sidedness of the right-mover global Ward identity is irrelevant and we may equivalently write the right-mover identity as

$$
C_{-}(\tilde{\mathcal{T}}, \sigma)\left(\sum_{\rho=1}^{N} \tilde{\mathcal{T}}_{0 \mu}^{(\rho)}\right)=0, \quad \forall \mu
$$

because $\mathcal{T}$ and $\tilde{\mathcal{T}}$ commute with the twisted vertex operators. Similarly, the constant factor $C_{-}(\tilde{\mathcal{T}}, \sigma)$ can be moved to the right of the $\bar{z}$-dependent factor in the twisted vertex operators. 


\section{- In terms of untilded quantities}

Using (2.82), (2.83) and (2.85), all the twisted right-mover results can be expressed in terms of the original untilded quantities $\hat{\bar{J}}, \mathcal{G}$ and $\mathcal{T}$. For example, one finds that

$$
\tilde{\mathcal{G}}^{n(r) \mu ;-n(r), \nu}(\sigma) \frac{\bar{n}(r)}{\rho(\sigma)} \tilde{\mathcal{T}}_{n(r) \mu} \tilde{\mathcal{T}}_{-n(r), \nu}=\mathcal{G}^{n(r) \mu ;-n(r), \nu}(\sigma) \frac{\bar{n}(r)}{\rho(\sigma)} \mathcal{T}_{n(r) \mu} \mathcal{T}_{-n(r), \nu}
$$

because the $\mathcal{T}$ 's commute and $\mathcal{G}$ is symmetric. It follows that, under $\bar{z} \rightarrow z$, the first factor in the twisted right-mover vertex operator (2.87b) is the same factor we found for the twisted left-mover vertex operators in (2.28a). As another example, we give the result for the right-mover orbifold correlators

$$
\begin{aligned}
& \hat{A}_{-}(\mathcal{T}, \bar{z}, \sigma)=C_{-}(-\mathcal{T}, \sigma)\left(\prod_{\rho} \bar{z}_{\rho}^{-\mathcal{G}^{n(r) \mu ;-n(r), \nu}(\sigma) \frac{\bar{n}(r)}{\rho(\sigma)} \mathcal{T}_{n(r) \mu}^{(\rho)} \mathcal{T}_{-n(r), \nu}^{(\rho)}}\right)\left(\prod_{\rho<\kappa} \bar{z}_{\rho \kappa}^{\mathcal{T}_{0 \mu}^{(\kappa)} \mathcal{G}^{0 \mu ; 0 \nu}(\sigma) \mathcal{T}_{0 \nu}^{(\rho)}}\right) \\
& \times \prod_{\rho<\kappa} \exp \left\{\mathcal{T}_{n(r) \mu}^{(\rho)} \mathcal{G}^{n(r) \mu ;-n(r), \nu}(\sigma) \mathcal{T}_{-n(r), \nu}^{(\kappa)}\left(1-\delta_{\bar{n}(r), 0}\right) I_{\frac{\bar{n}(r)}{\rho(\sigma)}}\left(\bar{z}_{\bar{z}_{\kappa}}, \infty\right)\right\} \\
& \left(\sum_{\rho=1}^{N} \mathcal{T}_{0 \mu}^{(\rho)}\right) C_{-}(-\mathcal{T}, \sigma)=C_{-}(-\mathcal{T}, \sigma)\left(\sum_{\rho=1}^{N} \mathcal{T}_{0 \mu}^{(\rho)}\right)=0, \quad \forall \mu, \quad \sigma=0, \ldots, N_{c}-1
\end{aligned}
$$

in each sector $\sigma$ of all $A_{\text {Cartan } g}(H) / H$.

\subsection{Full non-chiral results}

Combining the twisted left- and right-mover results above, we obtain the full twisted vertex operators $\hat{g}=\hat{g}_{-} \hat{g}_{+}$and their intrinsic monodromy in each sector $\sigma$ of $A_{\text {Cartan } g}(H) / H$ :

$$
\begin{gathered}
\hat{g}(\mathcal{T}, \bar{z}, z, \sigma)=\hat{g}_{-}(\mathcal{T}, \bar{z}, \sigma) \hat{g}_{+}(\mathcal{T}, z, \sigma), \quad \sigma=0, \ldots, N_{c}-1 \\
\hat{g}\left(\mathcal{T}, \bar{z} e^{-2 \pi i}, z e^{2 \pi i}, \sigma\right)=E(T, \sigma) \hat{g}(\mathcal{T}, \bar{z}, z, \sigma) E(T, \sigma)^{*} e^{2 \pi i\left(\hat{J}_{0 \mu}(0)+\hat{\bar{J}}_{0 \mu}(0)\right) \mathcal{G}^{0 \mu ; 0 \nu}(\sigma) \mathcal{T}_{0 \nu}} .
\end{gathered}
$$

Here we used Eqs. (2.33a, (2.88a $)$ and (2.91) to obtain the result (2.93b). Except for the last factor, which is a quantum correction, the monodromy 2.93b is consistent with the classical monodromy

$$
\hat{g}\left(\mathcal{T}, \bar{z} e^{-2 \pi i}, z e^{2 \pi i}, \sigma\right)=E(T, \sigma) \hat{g}(\mathcal{T}, \bar{z}, z, \sigma) E(T, \sigma)^{*}, \quad \sigma=0, \ldots, N_{c}-1
$$

obtained for classical group orbifold elements in Ref. [10].

For the full orbifold correlators $\hat{A}=\hat{A}_{-} \hat{A}_{+}$we obtain

$$
\begin{aligned}
& \hat{A}(\mathcal{T}, \bar{z}, z, \sigma)=\left\langle\hat{g}\left(\mathcal{T}^{(1)}, \bar{z}_{1}, z_{1}, \sigma\right) \cdots \hat{g}\left(\mathcal{T}^{(N)}, \bar{z}_{N}, z_{N}, \sigma\right)\right\rangle_{\sigma}, \quad \sigma=0, \ldots, N_{c}-1 \\
& =C(\mathcal{T}, \sigma) \prod_{\rho}\left|z_{\rho}\right|^{-2 \mathcal{G}^{n(r) \mu ;-n(r), \nu}(\sigma) \frac{\bar{n}(r)}{\rho(\sigma)}} \mathcal{T}_{n(r) \mu}^{(\rho)} \mathcal{T}_{-n(r), \nu}^{(\rho)} \prod_{\rho<\kappa}\left|z_{\rho \kappa}\right|^{2 \mathcal{T}_{0 \mu}^{(\kappa)} \mathcal{G}^{0 \mu ; 0 \nu}(\sigma) \mathcal{T}_{0 \nu}^{(\rho)}} \prod_{\rho<\kappa} e^{F(\rho, \kappa)}
\end{aligned}
$$




$$
\begin{aligned}
F(\rho, \kappa) \equiv & \mathcal{G}^{n(r) \mu ;-n(r), \nu}(\sigma)\left(1-\delta_{\bar{n}(r), 0}\right) \\
& \times\left(\mathcal{T}_{n(r) \mu}^{(\rho)} \mathcal{T}_{-n(r), \nu}^{(\kappa)} I_{\frac{\bar{n}(r)}{\rho(\sigma)}}\left(\frac{z_{\rho}}{z_{\kappa}}, \infty\right)+\mathcal{T}_{n(r) \mu}^{(\kappa)} \mathcal{T}_{-n(r), \nu}^{(\rho)} I_{\frac{\bar{n}(r)}{\rho(\sigma)}}\left(\frac{\bar{z}_{\rho}}{\bar{z}_{\kappa}}, \infty\right)\right) \\
C(\mathcal{T}, \sigma) \equiv C_{-}(-\mathcal{T}, \sigma) & C_{+}(\mathcal{T}, \sigma), \quad\left(\sum_{\rho=1}^{N} \mathcal{T}_{0 \mu}^{(\rho)}\right) C(\mathcal{T}, \sigma)=C(\mathcal{T}, \sigma)\left(\sum_{\rho=1}^{N} \mathcal{T}_{0 \mu}^{(\rho)}\right)=0
\end{aligned}
$$

where the function $F(\rho, \kappa)$ is defined only for $\rho \neq \kappa$ and the global Ward identities (2.95d) hold for all degeneracy indices $\mu$. As expected, the orbifold correlators (2.95) are symmetric under the exchange of any two full twisted vertex operators

$$
\hat{g}\left(\mathcal{T}^{(\rho)}, \bar{z}_{\rho}, z_{\rho}, \sigma\right) \leftrightarrow \hat{g}\left(\mathcal{T}^{(\kappa)}, \bar{z}_{\kappa}, z_{\kappa}, \sigma\right)
$$

This is checked explicitly in App. C, where the identities

$$
\begin{gathered}
I_{\frac{\bar{n}(r)}{\rho(\sigma)}}\left(y, y_{0}\right)=I_{\frac{\rho(\sigma)-\bar{n}(r)}{\rho(\sigma)}}\left(y^{-1}, y_{0}^{-1}\right), \quad I_{\frac{\bar{n}(r)}{\rho(\sigma)}}\left(y, y_{0}\right) \equiv \int_{y_{0}}^{y} \frac{d x}{x-1} x^{-\frac{\bar{n}(r)}{\rho(\sigma)}} \\
F(\kappa, \rho)=F(\rho, \kappa)+\Delta \\
\Delta=\mathcal{G}^{n(r) \mu ;-n(r), \nu}(\sigma)\left(1-\delta_{\bar{n}(r), 0}\right)\left(\mathcal{T}_{n(r) \mu}^{(\rho)} \mathcal{T}_{-n(r), \nu}^{(\kappa)}+\mathcal{T}_{n(r) \mu}^{(\kappa)} \mathcal{T}_{-n(r), \nu}^{(\rho)}\right) I_{\frac{\bar{n}(r)}{\rho(\sigma)}}(\infty, 0)=0 .
\end{gathered}
$$

are used to establish the required symmetry of $F$. The identity in (2.97a) was given in Ref. 10].

\subsection{Example: More about the inversion orbifold}

The twisted left-mover sector of the inversion orbifold $A_{\text {Cartan } \mathfrak{s u}(2)}\left(\mathbb{Z}_{2}\right) / \mathbb{Z}_{2}$ was studied in Subsec. 2.8. Here we illustrate the discussion above by providing the corresponding twisted rightmover and non-chiral results for this simple case.

Using (2.81c), (2.87b) and the data (2.69), (2.70) we find the twisted right-mover vertex operator equation and the twisted right-mover vertex operators

$$
\begin{gathered}
\bar{\partial} \hat{g}_{-}(\mathcal{T}, \bar{z})=-\frac{\mathcal{T}}{k}: \hat{\bar{J}}_{1}(\bar{z}) \hat{g}_{-}(\mathcal{T}, \bar{z}): \bar{M}-\frac{\mathcal{T}^{2}}{2 k \bar{z}} \hat{g}_{-}(\mathcal{T}, \bar{z}), \quad \mathcal{T}=\mathcal{T}(T)=-T_{2} \\
\hat{g}_{-}(\mathcal{T}, \bar{z})=\bar{z}^{-\frac{\mathcal{T}^{2}}{2 k}} \exp \left(\frac{\mathcal{T}}{k} \sum_{m \leq-1} \hat{\bar{J}}_{1}^{\mathrm{R}}\left(m+\frac{1}{2}\right) \frac{\bar{z}^{-\left(m+\frac{1}{2}\right)}}{m+\frac{1}{2}}\right) \exp \left(\frac{\mathcal{T}}{k} \sum_{m \geq 0} \hat{\bar{J}}_{1}^{\mathrm{R}}\left(m+\frac{1}{2}\right) \frac{\bar{z}^{-\left(m+\frac{1}{2}\right)}}{m+\frac{1}{2}}\right) .
\end{gathered}
$$

In this case, it is easy to check from Eq. 2.80b that the mode-reversed currents satisfy a copy of the twisted left-mover current algebra

$$
\left[\hat{\bar{J}}_{1}\left(m+\frac{1}{2}\right), \hat{\bar{J}}_{1}\left(n+\frac{1}{2}\right)\right]=-k\left(m+\frac{1}{2}\right) \delta_{m+n+1,0}
$$




$$
\begin{gathered}
\hat{\bar{J}}_{1}^{\mathrm{R}}\left(m+\frac{1}{2}\right)=\hat{\bar{J}}_{-1}\left(-m-\frac{1}{2}\right)=\hat{\bar{J}}_{1}\left(-m-1+\frac{1}{2}\right) \\
{\left[\hat{\bar{J}}_{1}^{\mathrm{R}}\left(m+\frac{1}{2}\right), \hat{\bar{J}}_{1}^{\mathrm{R}}\left(n+\frac{1}{2}\right)\right]=k\left(m+\frac{1}{2}\right) \delta_{m+n+1,0}}
\end{gathered}
$$

and hence that the mode-reversed currents provide a realization of the rectified right-mover currents $\hat{\bar{J}}^{\sharp}$ of Ref. [10].

Combining the result (2.98b) with the twisted left-mover vertex operators in (2.77b), we find the full twisted vertex operators $\hat{g}=\hat{g}_{-} \hat{g}_{+}$:

$$
\begin{aligned}
\hat{g}(\mathcal{T}, \bar{z}, z)= & |z|^{-\frac{\mathcal{T}^{2}}{k}} \exp \left(\frac{\mathcal{T}}{k} \sum_{m \leq-1} \frac{1}{m+\frac{1}{2}}\left(\hat{\bar{J}}_{1}^{\mathrm{R}}\left(m+\frac{1}{2}\right) \bar{z}^{-\left(m+\frac{1}{2}\right)}-\hat{J}_{1}\left(m+\frac{1}{2}\right) z^{-\left(m+\frac{1}{2}\right)}\right)\right) \\
& \times \exp \left(\frac{\mathcal{T}}{k} \sum_{m \geq 0} \frac{1}{m+\frac{1}{2}}\left(\hat{\bar{J}}_{1}^{\mathrm{R}}\left(m+\frac{1}{2}\right) \bar{z}^{-\left(m+\frac{1}{2}\right)}-\hat{J}_{1}\left(m+\frac{1}{2}\right) z^{\left.-\left(m+\frac{1}{2}\right)\right)}\right)\right. \\
= & |z|^{-\frac{\mathcal{T}^{2}}{k}}: e^{i \hat{\beta}^{1}(\bar{z}, z) \mathcal{T}}:_{M} \\
i \hat{\beta}^{1}(\bar{z}, z)= & \frac{1}{k} \sum_{m \in \mathbb{Z}} \frac{1}{m+\frac{1}{2}}\left(\hat{\bar{J}}_{1}^{\mathrm{R}}\left(m+\frac{1}{2}\right) \bar{z}^{-\left(m+\frac{1}{2}\right)}-\hat{J}_{1}\left(m+\frac{1}{2}\right) z^{-\left(m+\frac{1}{2}\right)}\right)
\end{aligned}
$$

Because the inversion orbifold has no zero twist class $\bar{n}(r)=0$, we find that the intrinsic monodromy of the full twisted vertex operators

$$
\begin{aligned}
& \hat{\beta}^{1}\left(\bar{z} e^{-2 \pi i}, z e^{2 \pi i}\right)=-\hat{\beta}^{1}(\bar{z}, z), \quad \mathcal{T}(T)=-E(T) \mathcal{T}(T) E(T)^{*} \\
& \hat{g}\left(\mathcal{T}, \bar{z} e^{-2 \pi i}, z e^{2 \pi i}\right)= E(T) \hat{g}(\mathcal{T}, \bar{z}, z) E(T)^{*} \\
& \leftrightarrow \hat{g}\left(\mathcal{T}, \bar{z} e^{-2 \pi i}, z e^{2 \pi i}\right)_{m^{\prime}}{ }^{m}=e^{i \pi\left(m^{\prime}-m\right)} \hat{g}(\mathcal{T}, \bar{z}, z)_{m^{\prime}}{ }^{m}, \quad\left|m^{\prime}\right|,|m| \leq j
\end{aligned}
$$

is in agreement with the classical monodromy (2.94) of the corresponding group orbifold elements.

In this connection we also use the results of Ref. [10] to give the explicit forms of the group elements $g(T)$, the eigengroup elements $\mathcal{G}(\mathcal{T})$ and the group orbifold elements $\hat{g}(\mathcal{T})$

$$
\begin{gathered}
g(T, \bar{z}, z)=e^{i \beta(\bar{z}, z) T_{3}}, \quad \beta(\bar{z}, z)^{\prime}=-\beta(\bar{z}, z) \\
g(T, \bar{z}, z)^{\prime}=W(T) g(T, \bar{z}, z) W^{\dagger}(T)=e^{i \beta(\bar{z}, z)^{\prime} T_{3}}=e^{-i \beta(\bar{z}, z) T_{3}} \\
\mathcal{G}(\mathcal{T}, \bar{z}, z)=U(T) g(T, \bar{z}, z) U^{\dagger}(T)=e^{i \beta(\bar{z}, z) \mathcal{T}}=e^{-i \beta(\bar{z}, z) T_{2}} \\
\mathcal{G}(T, \bar{z}, z)^{\prime}=E(T) \mathcal{G}(T, \bar{z}, z) E^{*}(T)
\end{gathered}
$$




$$
\begin{gathered}
\hat{g}(\mathcal{T}, \bar{z}, z)=e^{i \hat{\beta}(\bar{z}, z) \mathcal{T}}=e^{-i \hat{\beta}(\bar{z}, z) T_{2}} \\
\hat{\beta}\left(\bar{z} e^{-2 \pi i}, z e^{2 \pi i}\right)=-\hat{\beta}(\bar{z}, z), \quad \hat{g}\left(\mathcal{T}, \bar{z} e^{-2 \pi i}, z e^{2 \pi i}\right)=E(T) \hat{g}(\mathcal{T}, \bar{z}, z) E^{*}(T)
\end{gathered}
$$

in the classical theory of the inversion orbifold. Here the primed quantities are the responses to the inversion in the symmetric theory, and $W(T), U^{\dagger}(T)$ and $E(T)$ are given in Eqs. (2.74) and (2.76).

Continuing with the quantum theory, we give the right-mover and the full non-chiral correlators of the inversion orbifold:

$$
\begin{gathered}
\left\langle\hat{g}_{-}\left(\mathcal{T}^{(1)}, \bar{z}_{1}\right) \cdots \hat{g}_{-}\left(\mathcal{T}^{(N)}, \bar{z}_{N}\right)\right\rangle=\left(\prod_{\rho} \bar{z}_{\rho}^{-\frac{1}{2 k} \mathcal{T}^{(\rho)} \mathcal{T}^{(\rho)}}\right) \prod_{\rho<\kappa}\left(\frac{\sqrt{z_{\rho}}-\sqrt{\bar{z}_{\kappa}}}{\sqrt{\bar{z}_{\rho}}+\sqrt{\bar{z}_{\kappa}}}\right)^{\frac{\mathcal{T}^{(\kappa)} \mathcal{T}^{(\rho)}}{k}} \\
\left\langle\hat{g}\left(\mathcal{T}^{(1)}, \bar{z}_{1}, z_{1}\right) \cdots \hat{g}\left(\mathcal{T}^{(N)}, \bar{z}_{N}, z_{N}\right)\right\rangle=\left(\prod_{\rho}\left|z_{\rho}\right|^{-\frac{1}{k} \mathcal{T}^{(\rho)}} \mathcal{T}^{(\rho)}\right) \prod_{\rho<\kappa}\left|\frac{\sqrt{z_{\rho}}-\sqrt{z_{\kappa}}}{\sqrt{z_{\rho}}+\sqrt{z_{\kappa}}}\right|^{\frac{2}{k} \mathcal{T}^{(\kappa)} \mathcal{T}^{(\rho)}} .
\end{gathered}
$$

Note that the intrinsic monodromy (2.101b) of the full vertex operators is consistent with the form of the full correlators, e.g.

$$
\begin{aligned}
&\left\langle\hat{g}\left(\mathcal{T}^{(1)}, \bar{z}_{1} e^{-2 \pi i}, z_{1} e^{2 \pi i}\right) \cdots \hat{g}\left(\mathcal{T}^{(N)}, \bar{z}_{N} e^{-2 \pi i}, z_{N} e^{2 \pi i}\right)\right\rangle \\
&=\left\langle\hat{g}\left(\mathcal{T}^{(1)}, \bar{z}_{1}, z_{1}\right) \cdots \hat{g}\left(\mathcal{T}^{(N)}, \bar{z}_{N}, z_{N}\right)\right\rangle \\
&= E(\{T\})\left\langle\hat{g}\left(\mathcal{T}^{(1)}, \bar{z}_{1}, z_{1}\right) \cdots \hat{g}\left(\mathcal{T}^{(N)}, \bar{z}_{N}, z_{N}\right)\right\rangle E(\{T\})^{*} \\
& E(\{T\})=\otimes_{\rho=1}^{N} E\left(T^{(\rho)}\right), \quad E(\{T\})^{*}=\otimes_{\rho=1}^{N} E\left(T^{(\rho)}\right)^{*} .
\end{aligned}
$$

Here the equality (2.104a $)$ follows from the correlators $2.103 \mathrm{~b})$, while the equality (2.104b) follows from the monodromy (2.101) of the full twisted vertex operators. The agreement of the two forms follows from the $\mathcal{T}$-selection rule (2.101b) and the fact that the $\mathcal{T}$ 's appear quadratically in the correlators.

\section{The Charge Conjugation Orbifold on $\mathfrak{s u}(n)$}

\subsection{Charge conjugation}

As our second large example, we apply the general results of Ref. [10] to study the (outerautomorphic $f^{2}$ ) charge conjugation orbifold on $\mathfrak{s u}(n)$

$$
\frac{A_{\mathfrak{s u}(n)}\left(\mathbb{Z}_{2}\right)}{\mathbb{Z}_{2}}, \quad n \geq 3 .
$$

One can follow our development through for the charge conjugation orbifold $A_{\mathfrak{s u}(2)}\left(\mathbb{Z}_{2}\right) / \mathbb{Z}_{2}$ as well, but in this case the automorphism is an inner automorphism and all irreps are real representations.

\footnotetext{
${ }^{\ddagger}$ At the level of characters, useful references for outer-automorphically twisted affine Lie algebras include [19-22].
} 
For accessibility in physics we will work in the standard Cartesian basis of $\mathfrak{s u}(n)$, with generalized $n \times n$ Gell-Mann matrices $\lambda_{a}^{\dagger}=\lambda_{a}$ and real structure constants $f_{a b}{ }^{c}=f_{a b c}$

$$
\begin{aligned}
& {\left[\lambda_{a}, \lambda_{b}\right]=2 i F_{a b c} \lambda_{c}, \quad f_{a b c}=\sqrt{\psi^{2}} F_{a b c}, \quad G_{a b}=k \delta_{a b}} \\
& \operatorname{Tr}\left(\lambda_{a} \lambda_{b}\right)=2 \delta_{a b}, \quad \operatorname{Tr} \lambda_{a}=0, \quad a, b=1, \ldots, n^{2}-1
\end{aligned}
$$

where $\sqrt{\psi^{2}}$ is the root length of $\mathfrak{s u}(n)$ and $k$ is the level of affine $\mathfrak{s u}(n)$. The standard iterative scheme is assumed for the generalized Gell-Mann matrices of $\mathfrak{s u}(n \geq 4)$, so that e.g. $\lambda_{9}$ for $\mathfrak{s u}(4)$ has a 1 in the $(1,4)$ and $(4,1)$ entries.

In this basis, the OPEs of affine $\mathfrak{s u}(n)$ are

$$
\begin{aligned}
J_{A}(z) J_{B}(w) & =\frac{k \delta_{A B}}{(z-w)^{2}}+\frac{i f_{A B C} J_{C}(w)}{z-w}+\mathcal{O}(z-w)^{0} \\
J_{A}(z) J_{I}(w) & =\frac{i f_{A I J} J_{J}(w)}{z-w}+\mathcal{O}(z-w)^{0} \\
J_{I}(z) J_{J}(w) & =\frac{k \delta_{I J}}{(z-w)^{2}}+\frac{i f_{I J A} J_{A}(w)}{z-w}+\mathcal{O}(z-w)^{0} \\
A & \in h=\mathfrak{s o}(n), \quad I \in \frac{g}{h}=\frac{\mathfrak{s u}(n)}{\mathfrak{s o}(n)}
\end{aligned}
$$

where we have decomposed the affine algebra according to the symmetric space

$$
\frac{\mathfrak{s u}(n)_{x}}{\mathfrak{s o}(n)_{2 \tau x}}, \quad x=\frac{2 k}{\psi^{2}}, \quad \tau= \begin{cases}2 & \text { for } n=3 \\ 1 & \text { for } n \geq 4\end{cases}
$$

We will choose in particular the $\mathfrak{s o}(n)$ subalgebra which corresponds to the Cartan-Weyl generators $\left\{i\left(\tilde{E}_{\alpha}-\tilde{E}_{-\alpha}\right), \forall \alpha>0\right\}$, so that for example:

$$
\begin{aligned}
& \mathfrak{s u}(3) \quad: \quad A=2,5,7, \quad I=1,3,4,6,8 \\
& \mathfrak{s u}(4) \quad: \quad A=2,5,7,10,12,14, \quad I=1,3,4,6,8,9,11,13,15 .
\end{aligned}
$$

Then one finds for the fundamental representation of $\mathfrak{s u}(n)$

$$
T_{a}=\frac{\sqrt{\psi^{2}}}{2} \lambda_{a}, \quad \operatorname{Tr}\left(T_{a} T_{b}\right)=\frac{\psi^{2}}{2} \delta_{a b}, \quad T_{a}^{\dagger}=T_{a}, \quad T_{A}^{*}=-T_{A}, \quad T_{I}^{*}=T_{I}
$$

that the $\mathfrak{s o}(n)$ generators $\left\{T_{A}\right\}$ are proportional to the standard generators of Cartesian $\mathfrak{s o}(n)$ in the vector representation

$$
\begin{gathered}
\left(T_{i j}\right)_{k}^{l}=i \frac{\sqrt{\psi^{2}}}{2}\left(\delta_{i k} \delta_{j}^{l}-\delta_{j k} \delta_{i}^{l}\right)=i \sqrt{\frac{\tau \psi_{\mathfrak{s o}(n)}^{2}}{2}}\left(\delta_{i k} \delta_{j}^{l}-\delta_{j k} \delta_{i}^{l}\right) \\
1 \leq i<j \leq n, \quad k, l=1 \ldots n, \quad x_{\mathfrak{s o}(n)}=\frac{\psi^{2}}{\psi_{\mathfrak{s o}(n)}^{2}} x=2 \tau x
\end{gathered}
$$


where $T_{A} \leftrightarrow-T_{i<j}$ is a relabelling of each $n \times n$ matrix $T_{A}$ by its non-zero entries. The quantity $\psi_{\mathfrak{s o}(n)}$ is the highest root of $\mathfrak{s o}(n)$, and this choice of $\mathfrak{s o}(n)$ is inner-automorphically equivalent to any other $\mathfrak{s o}(n)$ subalgebra which is irregularly embedded at the level shown in (3.4).

Note in particular that the commuting Cartan generators of $\mathfrak{s u}(n)$

$$
\begin{gathered}
J_{\dot{a}}, \quad \dot{a} \in \operatorname{Cartan} \mathfrak{s u}(n), \quad \operatorname{Cartan} \mathfrak{s u}(n) \subset \frac{\mathfrak{s u}(n)}{\mathfrak{s o}(n)} \\
\mathfrak{s u}(3) \quad: \quad \dot{a}=3,8, \quad \mathfrak{s u}(4) \quad: \quad \dot{a}=3,8,15, \quad \mathfrak{s u}(5) \quad: \quad \dot{a}=3,8,15,24
\end{gathered}
$$

are always in $g / h$. In this notation, the general matrix irrep $T$ of $\mathfrak{s u}(n)$ satisfies

$$
\begin{gathered}
\left(T_{a}\right)_{\lambda^{i}}{ }^{\lambda^{j}}=\left\langle\lambda^{i}(T)\left|J_{a}(0)\right| \lambda^{j}(T)\right\rangle, \quad T_{a}^{\dagger}=T_{a} \\
{\left[J_{a}(0), J_{b}(0)\right]=i f_{a b c} J_{c}(0), \quad\left[T_{a}, T_{b}\right]=i f_{a b c} T_{c}} \\
\left(T_{\dot{a}}\right)_{\lambda^{i}}{ }^{\lambda^{j}}=\lambda_{\dot{a}}^{i}(T) \delta_{\lambda^{i}}^{\lambda^{j}}, \quad \dot{a} \in \operatorname{Cartan} \mathfrak{s u}(n) \subset \frac{\mathfrak{s u}(n)}{\mathfrak{s o}(n)}
\end{gathered}
$$

where $J_{a}(0)$ are the generators of $\mathfrak{s u}(n)$ and $\lambda^{i}(T), i=1 \ldots \operatorname{dim} T$ are the weights of irrep $T$.

We turn next to the non-trivial element $(\sigma=1)$ of the charge conjugation automorphism group, whose action on the currents is

$$
\begin{gathered}
J_{a}(z)^{\prime}=\omega_{a}^{b} J_{b}(z), \quad \omega^{\dagger}=\omega \\
\omega_{A}{ }^{A}=-\omega_{I}^{I}=1, \quad A \in \mathfrak{s o}(n), \quad I \in \mathfrak{s u}(n) / \mathfrak{s o}(n)
\end{gathered}
$$

where $\omega$ is a diagonal matrix whose diagonal elements are given in $(3.10 \mathrm{~b})$. Because $\mathfrak{s u}(n) / \mathfrak{s o}(n)$ is a symmetric space it is trivial to check that this is an automorphism of the affine OPEs (3.3), with invariant subalgebra $h=\mathfrak{s o}(n)$. To identify this automorphism as the Cartesian form of charge conjugation we consider for each irrep $T$ the following two closely-related representations of $\mathfrak{s u}(n)$

$$
\begin{gathered}
\bar{T}_{a} \equiv-T_{a}^{\mathrm{T}}, \quad T_{a}{ }^{\prime} \equiv \omega_{a}{ }^{b} T_{b} \\
{\left[\bar{T}_{a}, \bar{T}_{b}\right]=i f_{a b c} \bar{T}_{c}, \quad\left[T_{a}{ }^{\prime}, T_{b}{ }^{\prime}\right]=i f_{a b c} T_{c}{ }^{\prime}}
\end{gathered}
$$

where superscript $\mathrm{T}$ is matrix transpose, $\bar{T}$ is the standard charge conjugate representation of $T$ and $T^{\prime}$ is the automorphic transform of $T$. In fact, $T^{\prime}$ is unitarily equivalent to $\bar{T}$

$$
T^{\prime} \simeq \bar{T}
$$

because both representations have the weights

$$
\left(\bar{T}_{\dot{a}}\right)_{\lambda^{i}, \lambda^{j}}=\left(T_{\dot{a}}{ }^{\prime}\right)_{\lambda^{i}, \lambda^{j}}=-\lambda_{\dot{a}}^{i} \delta_{\lambda^{i}, \lambda^{j}}, \quad \dot{a} \in \operatorname{Cartan} \mathfrak{s u}(n)
$$


of the charge conjugate representation. Our Cartesian automorphism $\omega$ is inner-automorphically equivalent $\omega \simeq \tau$ to a realization of the action $\tau$ of the Dynkin automorphism. This is discussed explicitly for $\mathfrak{s u}(3)$ in App. D, which also remarks on the inner-automorphically equivalent realization of $\tau$ with invariant subalgebra $h=\mathfrak{c}_{n}$ for $\mathfrak{s u}(2 n)$.

Following the observation (3.12), we will say that a real representation of $\mathfrak{s u}(n)$ satisfies

$$
T \simeq \bar{T} \simeq T^{\prime}
$$

while for a complex representation $\bar{T}$ or $T^{\prime}$ is not equivalent to $T$. As examples, the adjoint representation is real with

$$
\left(T_{a}^{\mathrm{adj}}\right)_{b}{ }^{c}=-i f_{a b c}, \quad \bar{T}_{a}^{\mathrm{adj}}=T_{a}^{\mathrm{adj}} \simeq T_{a}^{\mathrm{adj} \prime}
$$

while the fundamental representation $n$ is complex with

$$
T_{a}=\frac{\sqrt{\psi^{2}}}{2} \lambda_{a}, \quad T_{A}^{\mathrm{T}}=-T_{A}, \quad T_{I}^{\mathrm{T}}=T_{I}, \quad T_{a}^{\prime}=\bar{T}_{a} .
$$

In this case the two equivalent matrix representations $T^{\prime}$ and $\bar{T}$ of $\bar{n}$ are exactly equal.

\subsection{The Cartesian form of $A_{n-1}^{(2)}$}

Because the action $\omega$ of charge conjugation is diagonal in the Cartesian basis, the solution to the $H$-eigenvalue problem is trivial

$$
\begin{array}{cl}
\omega U^{\dagger}=U^{\dagger} E \quad: & \sigma=1, \quad \rho=2, \quad U^{\dagger}=1, \quad E=\omega \\
\bar{n}(r)=0 & \leftrightarrow E_{A}=1, \quad \forall A \in \mathfrak{s o}(n) \\
\bar{n}(r)=1 \quad \leftrightarrow E_{I}=-1, \quad \forall I \in \mathfrak{s u}(n) / \mathfrak{s o}(n) .
\end{array}
$$

Then the normalization $\chi=1$ gives the eigencurrents $\mathcal{J}$ and the non-zero entries of the basic duality transformations

$$
\begin{gathered}
\mathcal{J}_{0 A}(z)=J_{A}(z), \quad \mathcal{J}_{1 I}(z)=J_{I}(z) \quad ; \quad \mathcal{J}_{0 A}(z)^{\prime}=\mathcal{J}_{0 A}(z), \quad \mathcal{J}_{1 I}(z)^{\prime}=-\mathcal{J}_{1 I}(z) \\
\mathcal{G}_{0 A ; 0 B}=k \delta_{A B}, \quad \mathcal{G}_{1 I ;-1, J}=k \delta_{I J} \\
\mathcal{F}_{0 A ; 0 B}{ }^{0 C}=f_{A B C}, \quad \mathcal{F}_{0 A ; 1 I}{ }^{1 J}=f_{A I J}, \quad \mathcal{F}_{1 I ;-1, J}{ }^{0 A}=f_{I J A}
\end{gathered}
$$

and the principle of local isomorphisms [3] gives the twisted current system:

$$
\begin{gathered}
\hat{J}_{n(r) \mu}=\left\{\hat{J}_{0 A}, \hat{J}_{1 I}\right\}, \quad \hat{J}_{n(r) \pm 2, \mu}(z)=\hat{J}_{n(r) \mu}(z), \quad \hat{J}_{n(r) \mu}\left(z e^{2 \pi i}\right)=e^{-2 \pi i \frac{n(r)}{\rho(\sigma)}} \hat{J}_{n(r) \mu}(z) \\
\hat{J}_{0 A}(z) \hat{J}_{0 B}(w)=\frac{k \delta_{A B}}{(z-w)^{2}}+\frac{i f_{A B C} \hat{J}_{0 C}(w)}{z-w}+\mathcal{O}(z-w)^{0}
\end{gathered}
$$




$$
\begin{gathered}
\hat{J}_{0 A}(z) \hat{J}_{1 I}(w)=\frac{i f_{A I J} \hat{J}_{1 J}(w)}{z-w}+\mathcal{O}(z-w)^{0} \\
\hat{J}_{1 I}(z) \hat{J}_{1 J}(w)=\frac{k \delta_{I J}}{(z-w)^{2}}+\frac{i f_{I J A} \hat{J}_{2 A}(w)}{z-w}+\mathcal{O}(z-w)^{0} \\
\hat{J}_{0 A}\left(z e^{2 \pi i}\right)=\hat{J}_{0 A}(z), \quad \hat{J}_{1 I}\left(z e^{2 \pi i}\right)=-\hat{J}_{1 I}(z), \quad A \in \mathfrak{s o}(n), \quad I \in \mathfrak{s u}(n) / \mathfrak{s o}(n) .
\end{gathered}
$$

Finally the monodromies (3.19]) give the twisted mode expansions

$$
\hat{J}_{0 A}(z)=\sum_{m \in \mathbb{Z}} \hat{J}_{0 A}(m) z^{-m-1}, \quad \hat{J}_{1 I}(z)=\sum_{m \in \mathbb{Z}} \hat{J}_{1 I}\left(m+\frac{1}{2}\right) z^{-\left(m+\frac{1}{2}\right)-1}
$$

and one finds from (3.19), (3.20) that the twisted modes satisfy the Cartesian form of the outerautomorphically twisted affine Lie algebra $A_{n-1}^{(2)}$ :

$$
\begin{gathered}
{\left[\hat{J}_{0 A}(m), \hat{J}_{0 B}(n)\right]=i f_{A B C} \hat{J}_{0 C}(m+n)+k m \delta_{A B} \delta_{m+n, 0}} \\
{\left[\hat{J}_{0 A}(m), \hat{J}_{1 I}\left(n+\frac{1}{2}\right)\right]=i f_{A I J} \hat{J}_{1 J}\left(m+n+\frac{1}{2}\right)} \\
{\left[\hat{J}_{1 I}\left(m+\frac{1}{2}\right), \hat{J}_{1 J}\left(n+\frac{1}{2}\right)\right]=i f_{I J A} \hat{J}_{2 A}(m+n+1)+k\left(m+\frac{1}{2}\right) \delta_{I J} \delta_{m+n+1,0}} \\
\hat{J}_{ \pm 2, A}(m)=\hat{J}_{0 A}(m), \quad \hat{J}_{-1, I}\left(m-\frac{1}{2}\right)=\hat{J}_{1 I}\left(m-1+\frac{1}{2}\right) \\
\hat{J}_{0 A}(m)^{\dagger}=\hat{J}_{0 A}(-m), \quad \hat{J}_{1 I}\left(m+\frac{1}{2}\right)^{\dagger}=\hat{J}_{-1, I}\left(-m-\frac{1}{2}\right)=\hat{J}_{1 I}\left(-m-1+\frac{1}{2}\right) \\
A \in \mathfrak{s o}(n), \quad I \in \mathfrak{s u}(n) / \mathfrak{s o}(n) .
\end{gathered}
$$

This twisted current algebra (and (3.19)) can alternately be obtained by substitution of the data (3.17), (3.18) into the general twisted current algebra of Ref. [四], and the dagger relations in (3.21e) follow from the general orbifold adjoint operation [4 in this case. App. D gives a translation dictionary between our Cartesian form of $A_{2}^{(2)}$ and its more conventional form in mathematics.

We also note that for even levels the outer-automorphically twisted affine Lie algebra (3.21) is a subalgebra of the order-two orbifold affine algebra [1] on $\mathfrak{s u}(n)$

$$
\begin{gathered}
{\left[\hat{J}_{a}^{(r)}\left(m+\frac{r}{2}\right), \hat{J}_{b}^{(s)}\left(n+\frac{s}{2}\right)\right]=i f_{a b c} \hat{J}_{c}^{(r+s)}\left(m+n+\frac{r+s}{2}\right)+\hat{k} \delta_{a b}\left(m+\frac{r}{2}\right) \delta_{m+n+\frac{r+s}{2}, 0}} \\
\hat{k}=2 k, \quad \bar{r}, \bar{s}=0,1, \quad a, b, c=1, \ldots, n^{2}-1
\end{gathered}
$$

which lives in the twisted sector of $\mathbb{Z}_{2}$ cyclic permutation orbifolds on $\mathfrak{s u}(n)_{k} \oplus \mathfrak{s u}(n)_{k}$.

Similarly, one reads from Ref. [4] the twisted left-mover affine-Sugawara construction of sector $\sigma=1$

$$
\begin{aligned}
\mathcal{L}_{\hat{\mathfrak{g}}}^{0 A ; 0 B}=\frac{1}{2 k+Q_{g}} \delta^{A B}, \quad \mathcal{L}_{\hat{\mathfrak{g}}}^{1 I ;-1, J}=\frac{1}{2 k+Q_{g}} \delta^{I J}, \quad \hat{c}=c=\frac{x\left(n^{2}-1\right)}{x+n} \\
\hat{T}(z)=\mathcal{L}_{\hat{\mathfrak{g}}}^{n(r) \mu ;-n(r), \nu}: \hat{J}_{n(r) \mu}(z) \hat{J}_{-n(r), \nu}(z): \\
=\frac{1}{2 k+Q_{g}}\left(: \sum_{A} \hat{J}_{0 A}(z) \hat{J}_{0 A}(z)+\sum_{I} \hat{J}_{1 I}(z) \hat{J}_{-1, I}(z):\right)
\end{aligned}
$$

where $\hat{\mathfrak{g}} \equiv \hat{\mathfrak{g}}(\sigma=1), Q_{g}$ is the quadratic Casimir of $\mathfrak{s u}(n)$ and : $:$ : is operator product normal ordering. 


\subsection{The twisted representation matrices}

To study the representation theory of the charge conjugation orbifold in the twisted sector, we need to determine the twisted representation matrices $\mathcal{T}(T)$ corresponding to each untwisted representation $T$.

In order to do this, we must first find the action $W(T)$ of the automorphism in representation $T$, which satisfies the linkage relation [10]

$$
W^{\dagger}(T) T_{a} W(T)=T_{a}{ }^{\prime}=\omega_{a}^{b} T_{b}
$$

and then we must solve the extended $H$-eigenvalue problem 10] for $W(T)$

$$
W(T) U^{\dagger}(T)=U^{\dagger}(T) E(T) .
$$

Given this solution (see below) and the eigendata (3.17) of the $H$-eigenvalue problem, we read from Ref. [10] the form and properties of the twisted representation matrices $\mathcal{T}$ :

$$
\begin{aligned}
\mathcal{T}_{0 A}(T)=\mathcal{T}_{ \pm 2, A}(T)=U(T) T_{A} U^{\dagger}(T), & \mathcal{T}_{1 I}(T)=\mathcal{T}_{-1, I}(T)=U(T) T_{I} U^{\dagger}(T) \\
{\left[\mathcal{T}_{0 A}(T), \mathcal{T}_{0 B}(T)\right] } & =i f_{A B C} \mathcal{T}_{0 C}(T) \\
{\left[\mathcal{T}_{0 A}(T), \mathcal{T}_{1 I}(T)\right] } & =i f_{A I J} \mathcal{T}_{1 J}(T) \\
{\left[\mathcal{T}_{1 I}(T), \mathcal{T}_{1 J}(T)\right] } & =i f_{I J A} \mathcal{T}_{0 A}(T) \\
\mathcal{T}_{0 A}(T)=E(T) \mathcal{T}_{0 A}(T) E(T)^{*}, & \mathcal{T}_{1 I}(T)=-E(T) \mathcal{T}_{1 I}(T) E(T)^{*}
\end{aligned}
$$

Here the relations $(3.26 \mathrm{e})$ are the $\mathcal{T}$-selection rules, which are the orbifold dual of the linkage relation.

To go further we must distinguish between real and complex representations of $\mathfrak{s u}(n)$. For any real representation, the solution $W(T)$ of the linkage relation (3.24) is guaranteed because

$T^{\prime} \simeq T$ for real representations. As an example, we know 10 for the adjoint representation $\left(T_{a}^{\mathrm{adj}}\right)_{b}{ }^{c}=-i f_{a b}{ }^{c}$ :

$$
\begin{gathered}
W\left(T^{\mathrm{adj}}\right)=\omega, \quad U\left(T^{\mathrm{adj}}\right)=U=1, \quad E\left(T^{\mathrm{adj}}\right)=E=\omega, \quad R\left(T^{\mathrm{adj}}\right)=\rho=2 \\
W^{\dagger}\left(T^{\mathrm{adj}}\right) T_{a}^{\mathrm{adj}} W\left(T^{\mathrm{adj}}\right)=\omega_{a}{ }^{b} T_{b}^{\mathrm{adj}} \leftrightarrow \omega_{a}{ }^{d} \omega_{b}{ }^{e} f_{d e}{ }^{f}\left(\omega^{\dagger}\right) f^{c}=f_{a b}{ }^{c} .
\end{gathered}
$$

Indeed, the relation in (3.27b) reminds us [10] that $W\left(T^{\mathrm{adj}}\right)=\omega$ for the adjoint in all currentalgebraic orbifolds. Using formulae (3.26a) above, we find the twisted representation matrices for the adjoint

$$
\mathcal{T}_{0 A}\left(T^{\mathrm{adj}}\right)=T_{A}^{\mathrm{adj}}, \quad \mathcal{T}_{1 I}\left(T^{\mathrm{adj}}\right)=T_{I}^{\mathrm{adj}}
$$

The selection rules (3.26e) are easily checked explicitly in this case, for example

$$
\mathcal{T}_{1 I}\left(T^{\mathrm{adj}}\right)_{J}{ }^{K}=-\mathcal{T}_{1 I}\left(T^{\mathrm{adj}}\right)_{J}{ }^{K}
$$

is satisfied as $0=0$ because $f_{I J K}=0$ for a symmetric space. 
For complex representations $T^{(\mathrm{c})}$ of $\mathfrak{s u}(n)$, we choose the reducible representation 10]

$$
\begin{gathered}
T_{a}=\left(\begin{array}{cc}
T_{a}^{(\mathrm{c})} & 0 \\
0 & T_{a}^{(\mathrm{c}),}
\end{array}\right) \\
T_{a}^{\prime}=\omega_{a}{ }^{b} T_{b}=\left(\begin{array}{cc}
T_{a}^{(\mathrm{c}) \prime} & 0 \\
0 & T_{a}^{(\mathrm{c})}
\end{array}\right)
\end{gathered}
$$

where $T^{(\mathrm{c}) \prime}$ in Eqs. (3.10), (3.11) is the automorphic transform of $T^{(\mathrm{c})}$. The choice in (3.30a) results in considerable simplification over the unitarily-equivalent choice with $T^{(\mathrm{c}) \prime} \rightarrow \bar{T}^{(\mathrm{c})}$. In particular we then find for all complex representations that the solution of the linkage relation (3.24) is very simple

$$
W(T)=\exp \left(i \frac{\pi}{2}\left(\begin{array}{cc}
0 & 1 \\
1 & 0
\end{array}\right)\right)=i\left(\begin{array}{cc}
0 & 1 \\
1 & 0
\end{array}\right), \quad W^{\dagger}(T) W(T)=\left(\begin{array}{cc}
1 & 0 \\
0 & 1
\end{array}\right) .
$$

Moreover, the extended $H$-eigenvalue problem (3.25) is also easy to solve

$$
U(T)=U^{\dagger}(T)=\frac{1}{\sqrt{2}}\left(\begin{array}{cc}
1 & 1 \\
1 & -1
\end{array}\right), \quad U^{\dagger}(T) U(T)=\left(\begin{array}{cc}
1 & 0 \\
0 & 1
\end{array}\right), \quad E(T)=i\left(\begin{array}{cc}
1 & 0 \\
0 & -1
\end{array}\right) .
$$

Then we find for the twisted representation matrices

$$
\begin{aligned}
& \mathcal{T}_{0 A}(T)=U(T)\left(\begin{array}{cc}
T_{A}^{(\mathrm{c})} & 0 \\
0 & T_{A}^{(\mathrm{c}) \prime}
\end{array}\right) U^{\dagger}(T)=\left(\begin{array}{cc}
T_{A}^{(\mathrm{c})} & 0 \\
0 & T_{A}^{(\mathrm{c})}
\end{array}\right) \\
& \mathcal{T}_{1 I}(T)=U(T)\left(\begin{array}{cc}
T_{I}^{(\mathrm{c})} & 0 \\
0 & T_{I}^{(\mathrm{c}) \prime}
\end{array}\right) U^{\dagger}(T)=\left(\begin{array}{cc}
0 & T_{I}^{(\mathrm{c})} \\
T_{I}^{(\mathrm{c})} & 0
\end{array}\right)
\end{aligned}
$$

and these explicit forms allow us to verify that the $\mathcal{T}$-selection rules $(3.26 \mathrm{e})$ are identities.

\subsection{Twisted left-mover KZ system for charge conjugation orbifolds}

We consider here only the $\mathfrak{s o}(n)$-invariant or scalar twist-field state, which satisfies

$$
\begin{gathered}
\hat{J}_{0 A}(m \geq 0)|0\rangle=\langle 0| \hat{J}_{0 A}(m \leq 0)=0, \quad A \in \mathfrak{s o}(n) \\
\hat{J}_{1 I}\left(m+\frac{1}{2} \geq 0\right)|0\rangle=\langle 0| \hat{J}_{1 I}\left(m+\frac{1}{2} \leq 0\right)=0, \quad I \in \mathfrak{s u}(n) / \mathfrak{s o}(n)
\end{gathered}
$$

in the twisted sector of the charge conjugation orbifold. Then the $M$-ordered (mode-ordered) expressions of Refs. [4, 10] are immediately useful. For example, the left-mover Virasoro generators and the conformal weight of the scalar twist-field state

$$
\begin{gathered}
L_{\sigma}(m)=\frac{1}{2 k+Q_{g}} \sum_{p \in \mathbb{Z}}: \sum_{A} \hat{J}_{0 A}(p) \hat{J}_{0 A}(m-p)+\sum_{I} \hat{J}_{1 I}\left(p+\frac{1}{2}\right) \hat{J}_{-1, I}\left(m-p-\frac{1}{2}\right):_{M} \\
+\delta_{m, 0} \hat{\Delta}_{0}, \quad\left[L_{\sigma}(m \geq 0)-\delta_{m, 0} \hat{\Delta}_{0}\right]|0\rangle=0
\end{gathered}
$$




$$
\begin{aligned}
\hat{\Delta}_{0} & =\mathcal{L}_{\hat{\mathfrak{g}}(\sigma)}^{n(r) \mu ;-n(r) \nu}(\sigma) \frac{\bar{n}(r)}{2 \rho(\sigma)}\left(1-\frac{\bar{n}(r)}{\rho(\sigma)}\right) \mathcal{G}_{n(r) \mu ;-n(r) \nu}(\sigma) \\
& =\frac{\operatorname{dim}(g / h)}{16} \frac{x}{x+n}=\frac{(n-1)(n+2)}{32} \frac{x}{x+n}
\end{aligned}
$$

are easily read from (3.17), (3.18), (3.23) and the general form given in Eq. (6.8b) of Ref. [10].

We turn next to the left-mover twisted affine primary fields $\hat{g}_{+}(\mathcal{T}, z), \mathcal{T}=\mathcal{T}(T)$ of the charge conjugation orbifold. From Ref. [10], we read the OPEs

$$
\begin{gathered}
\hat{J}_{0 A}(z) \hat{g}_{+}(\mathcal{T}, w)=\frac{\hat{g}_{+}(\mathcal{T}, w)}{z-w} \mathcal{T}_{0 A}(T)+\mathcal{O}(z-w)^{0} \\
\hat{J}_{1 I}(z) \hat{g}_{+}(\mathcal{T}, w)=\frac{\hat{g}_{+}(\mathcal{T}, w)}{z-w} \mathcal{T}_{1 I}(T)+\mathcal{O}(z-w)^{0} \\
\hat{T}(z) \hat{g}_{+}(\mathcal{T}, w)=\left(\frac{\Delta}{(z-w)^{2}}+\frac{1}{z-w} \partial_{w}\right) \hat{g}_{+}(\mathcal{T}, w)+\mathcal{O}(z-w)^{0} \\
\Delta \equiv\left\{\begin{aligned}
\Delta(T) & \text { for real reps } T \\
\Delta\left(T^{(\mathrm{c})}\right)\left(\begin{array}{ll}
1 & 0 \\
0 & 1
\end{array}\right) & \text { for complex reps } T^{(\mathrm{c})}
\end{aligned}\right.
\end{gathered}
$$

where $\Delta(T)$ and $\Delta\left(T^{(\mathrm{c})}\right)$ are the conformal weight of the representation under the (untwisted) affine-Sugawara construction [5, 6, 23, 24] on $\mathfrak{s u}(n)$.

Similarly, using (3.17a), (3.23a) and Eq. (6.13a) of Ref. [10], we find the twisted left-mover vertex operator equation

$$
\begin{aligned}
\partial \hat{g}_{+}(\mathcal{T}, z)=\frac{2}{2 k+Q_{g}} & {\left[\sum_{A}: \hat{J}_{0 A}(z) \hat{g}_{+}(\mathcal{T}, z):_{M} \mathcal{T}_{0 A}\right.} \\
& \left.+\sum_{I}\left(: \hat{J}_{1 I}(z) \hat{g}_{+}(\mathcal{T}, z):_{M}-\frac{1}{2 z} \hat{g}_{+}(\mathcal{T}, z) \mathcal{T}_{1 I}\right) \mathcal{T}_{1 I}\right]
\end{aligned}
$$

for the twisted affine primary fields. $M$-ordering for the twisted vertex operator equation is defined in Ref. [10] (see also Eq. (2.16)). In combination with the twisted current algebra (3.21) and the ground state conditions (3.34), the twisted vertex operator equation (3.37) suffices to derive a twisted KZ system for the twisted sector of the charge conjugation orbifolds.

We prefer however to include this computation as an example of a more general result. For any twisted current algebra with an action on a general scalar twist-field state $|0\rangle_{\sigma}$

$$
\hat{J}_{n(r) \mu}\left(m+\frac{n(r)}{\rho(\sigma)} \geq 0\right)|0\rangle_{\sigma}={ }_{\sigma}\langle 0| \hat{J}_{n(r) \mu}\left(m+\frac{n(r)}{\rho(\sigma)} \leq 0\right)=0
$$

one finds that the general expression for the conformal weight $\hat{\Delta}_{0}(\sigma)$ of the scalar twist-field state is that given in Eq. (3.35b). Moreover, we may use the general twisted current algebra and the 
general twisted vertex operator equation of Ref. [10] to find the general twisted left-mover KZ system

$$
\begin{gathered}
\hat{A}_{+}(\mathcal{T}, z, \sigma) \equiv{ }_{\sigma}\left\langle 0\left|\hat{g}_{+}\left(\mathcal{T}^{(1)}, z_{1}, \sigma\right) \hat{g}_{+}\left(\mathcal{T}^{(2)}, z_{2}, \sigma\right) \cdots \hat{g}_{+}\left(\mathcal{T}^{(N)}, z_{N}, \sigma\right)\right| 0\right\rangle_{\sigma} \\
\partial_{\kappa} \hat{A}_{+}(\mathcal{T}, z, \sigma)=\hat{A}_{+}(\mathcal{T}, z, \sigma) \hat{W}_{\kappa}(\mathcal{T}, z, \sigma), \quad, \quad \kappa=1 \ldots N, \quad \sigma=0, \ldots, N_{c}-1 \\
\hat{W}_{\kappa}(\mathcal{T}, z, \sigma)=2 \mathcal{L}_{\hat{\mathfrak{g}}(\sigma)}^{n(r) \mu ;-n(r), \nu}(\sigma)\left[\sum_{\rho \neq \kappa}\left(\frac{z_{\rho}}{z_{\kappa}}\right)^{\frac{\bar{n}(r)}{\rho(\sigma)}} \frac{1}{z_{\kappa \rho}} \mathcal{T}_{n(r) \mu}^{(\rho)} \mathcal{T}_{-n(r), \nu}^{(\kappa)}-\frac{\bar{n}(r)}{\rho(\sigma)} \frac{1}{z_{\kappa}} \mathcal{T}_{n(r) \mu}^{(\kappa)} \mathcal{T}_{-n(r), \nu}^{(\kappa)}\right] \\
\hat{A}_{+}(\mathcal{T}, z, \sigma)\left(\sum_{\rho=1}^{N} \mathcal{T}_{0 \mu}^{(\rho)}\right)=0, \quad \forall \mu
\end{gathered}
$$

for the correlators in the scalar twist-field states of any WZW orbifold. The global Ward identity (3.39d) follows from the residual symmetry

$$
{ }_{\sigma}\left\langle 0\left|\left[\hat{J}_{0 \mu}(0), \hat{g}_{+}\left(\mathcal{T}^{(1)}, z_{1}, \sigma\right) \cdots \hat{g}_{+}\left(\mathcal{T}^{(N)}, z_{N}, \sigma\right)\right]\right| 0\right\rangle_{\sigma}=0, \quad \forall \mu
$$

generated by the zero modes of the integral affine subalgebra of the general twisted current algebra. The so-called ground state [4, 10] in sector $\sigma$ of any WZW permutation orbifold is in fact a scalar twist-field state, so the general twisted KZ system (3.39) includes as a special case the twisted KZ system obtained for the WZW permutation orbifolds in Ref. [10].

Moreover, a scalar twist-field state exists for each twisted sector of every outer-automorphically twisted affine Lie algebra, so the general system (3.39) includes a twisted KZ system for every outer-automorphic WZW orbifold. In these cases, the residual symmetry expressed by the global Ward identity (3.39d) is that of the invariant subalgebra $h \subset g$ of each automorphism group.

In particular, we obtain the twisted KZ system for the left-mover sector of the charge conjugation orbifold on $\mathfrak{s u}(n)$

$$
\begin{gathered}
\hat{A}_{+}(\mathcal{T}, z) \equiv\left\langle 0\left|\hat{g}_{+}\left(\mathcal{T}^{(1)}, z_{1}\right) \cdots \hat{g}_{+}\left(\mathcal{T}^{(N)}, z_{N}\right)\right| 0\right\rangle \\
\partial_{\mu} \hat{A}_{+}(\mathcal{T}, z)=\hat{A}_{+}(\mathcal{T}, z) \hat{W}_{\mu}(\mathcal{T}, z), \quad \mu=1 \ldots N \\
\hat{W}_{\mu}(\mathcal{T}, z)=\frac{2}{2 k+Q_{g}}\left[\sum_{\nu \neq \mu} \frac{1}{z_{\mu \nu}}\left(\sum_{A} \mathcal{T}_{0 A}^{(\nu)} \mathcal{T}_{0 A}^{(\mu)}+\left(\frac{z_{\nu}}{z_{\mu}}\right)^{\frac{1}{2}} \sum_{I} \mathcal{T}_{1 I}^{(\nu)} \mathcal{T}_{1 I}^{(\mu)}\right)\right. \\
\left.-\frac{1}{2 z_{\mu}} \sum_{I} \mathcal{T}_{1 I}^{(\mu)} \mathcal{T}_{1 I}^{(\mu)}\right] \\
\hat{A}_{+}(\mathcal{T}, z)\left(\sum_{\mu=1}^{N} \mathcal{T}_{0 A}^{(\mu)}\right)=0, \quad \forall A \in \mathfrak{s o}(n)
\end{gathered}
$$


where $|0\rangle$ is the $\mathfrak{s o}(n)$-invariant twist-field state in (3.34). For this case, the explicit form of the twisted representation matrices $\mathcal{T}=\mathcal{T}(T)$ is given in Subsec. 3.3. Although it is guaranteed by the construction, we check explicitly in App. E that the twisted connection (3.41d) is flat.

We emphasize here that the flatness check of App. E uses only the symmetric-space form $\left(f_{I J K}=0\right)$ of the algebra $\left.3.26 \mathrm{~b}\right),(3.26 \mathrm{~d}),(3.26 \mathrm{~d})$ of the twisted representation matrices. All $\mathbb{Z}_{2}$-type outer automorphism groups are similarly associated to symmetric spaces, so we expect that the twisted $\mathrm{KZ}$ systems of all $\mathbb{Z}_{2}$-type outer-automorphic orbifolds will have the same form (with $\mathfrak{s o}(n)$ replaced by the relevant invariant subalgebra) as that given in (3.41).

\subsection{Rectification and the twisted right-mover $\mathrm{KZ}$ system}

The form of the general twisted right-mover current algebra was given in Ref. [10]: As an example of the general phenomenon discussed there, we know that the twisted right-mover modes $\hat{\bar{J}}_{0 A}(m)$ and $\hat{\bar{J}}_{1 I}\left(m+\frac{1}{2}\right)$ satisfy the same algebra as $A_{n-1}^{(2)}$ in $(3.21)$, but now with $k \rightarrow-k$. This situation is rectifiable

$$
\hat{\bar{J}}_{0 A}^{\sharp}(m) \equiv \hat{\bar{J}}_{0 A}(-m), \quad \hat{\bar{J}}_{1 I}^{\sharp}\left(m+\frac{1}{2}\right) \equiv \hat{\bar{J}}_{-1, I}\left(-m-\frac{1}{2}\right)=\hat{\bar{J}}_{1 I}\left(-m-1+\frac{1}{2}\right) .
$$

The rectified right-mover modes $\hat{\bar{J}}^{\sharp}$ also satisfy a copy of the left-mover conditions

$$
\hat{\bar{J}}_{0 A}^{\sharp}(m \geq 0)|0\rangle=\left\langle 0\left|\hat{\bar{J}}_{0 A}^{\sharp}(m \leq 0)=0, \quad \hat{\bar{J}}_{1 I}^{\sharp}\left(m+\frac{1}{2} \geq 0\right)\right| 0\right\rangle=\langle 0| \hat{\bar{J}}_{1 I}^{\sharp}\left(m+\frac{1}{2} \leq 0\right)=0
$$

on the scalar twist-field state, and we find that the right-mover Virasoro generators are also a copy of the left movers

$$
\begin{gathered}
\bar{L}_{\sigma}(m)=\frac{1}{2 k+Q_{g}} \sum_{p \in \mathbb{Z}}: \sum_{A} \hat{\bar{J}}_{0 A}^{\sharp}(p) \hat{\bar{J}}_{0 A}^{\sharp}(m-p)+\sum_{I} \hat{\bar{J}}_{1 I}^{\sharp}\left(p+\frac{1}{2}\right) \hat{\bar{J}}_{-1, I}^{\sharp}\left(m-p-\frac{1}{2}\right): M \\
+\delta_{m, 0} \hat{\bar{\Delta}}_{0}, \quad \hat{\bar{c}}=\hat{c}=\frac{x\left(n^{2}-1\right)}{x+n} \\
{\left[\bar{L}_{\sigma}(m \geq 0)-\delta_{m, 0} \hat{\bar{\Delta}}_{0}\right]|0\rangle=0, \quad \hat{\bar{\Delta}}_{0}=\hat{\Delta}_{0}=\frac{(n-1)(n+2)}{32} \frac{x}{x+n}}
\end{gathered}
$$

with the same action on the scalar twist-field state.

Following Ref. [10], we also find the twisted KZ system for the right movers

$$
\begin{gathered}
\hat{A}_{-}(\mathcal{T}, \bar{z}) \equiv\left\langle 0\left|\hat{g}_{-}\left(\mathcal{T}^{(1)}, \bar{z}_{1}\right) \cdots \hat{g}_{-}\left(\mathcal{T}^{(N)}, \bar{z}_{N}\right)\right| 0\right\rangle \\
\bar{\partial}_{\mu} \hat{A}_{-}(\mathcal{T}, \bar{z})=\hat{\bar{W}}_{\mu}(\mathcal{T}, \bar{z}) \hat{A}_{-}(\mathcal{T}, \bar{z}), \quad \mu=1 \ldots N
\end{gathered}
$$

\footnotetext{
${ }^{\ddagger}$ The rectification problem is the question whether the twisted right- and left-mover current algebras are isomorphic. The question has been answered in the affirmative for inner-automorphic orbifolds on simple $g$ and for permutation orbifolds in Ref. [10]. Except for the triality orbifold on $\mathfrak{s o}(8)$, all outer automorphism groups of simple $g$ are $\mathbb{Z}_{2}$ 's, and we know that the non-trivial element of $\mathbb{Z}_{2}$ satisfies $h_{1}^{-1}=h_{1}$. According to the discussion of Ref. [10] this means that the twisted right-mover current algebra of all these orbifolds is similarly rectifiable, as seen here for the charge conjugation orbifold on $\mathfrak{s u}(n)$. Thus the rectification problem is solved except for the triality orbifold on $\mathfrak{s o}(8)$, to which we expect to return elsewhere.
} 


$$
\begin{gathered}
\hat{\bar{W}}_{\mu}(\mathcal{T}, \bar{z})=\frac{2}{2 k+Q_{g}}\left[\sum_{\nu \neq \mu} \frac{1}{\bar{z}_{\mu \nu}}\left(\sum_{A} \mathcal{T}_{0 A}^{(\mu)} \mathcal{T}_{0 A}^{(\nu)}+\left(\frac{\bar{z}_{\nu}}{\bar{z}_{\mu}}\right)^{\frac{1}{2}} \sum_{I} \mathcal{T}_{1 I}^{(\mu)} \mathcal{T}_{1 I}^{(\nu)}\right)\right. \\
\left.-\frac{1}{2 \bar{z}_{\mu}} \sum_{I} \mathcal{T}_{1 I}^{(\mu)} \mathcal{T}_{1 I}^{(\mu)}\right] \\
\left(\sum_{\mu=1}^{N} \mathcal{T}_{0 A}^{(\mu)}\right) \hat{A}_{-}(\mathcal{T}, \bar{z})=0, \quad \forall A \in \mathfrak{s o}(n) .
\end{gathered}
$$

In this case $\hat{\bar{W}}_{\mu}$ is just $\hat{W}_{\mu}$ with $\left\{z_{\mu}\right\} \rightarrow\left\{\bar{z}_{\mu}\right\}$ because the order of the matrices is reversible.

\subsection{The classical theory of charge conjugation orbifolds}

The classical action formulation of each sector of all WZW orbifolds, in terms of appropriate group orbifold elements with definite monodromy, was given in Ref. [10]. The group orbifold elements are the classical limit of the twisted affine primary fields of the WZW orbifolds. Before discussing solutions of our twisted KZ systems above, it will be helpful to work out the details of this classical formulation in the case of the charge conjugation orbifolds.

Reading from Subsec. 5.7 of Ref. [10], we find for all representations that the group elements $g$, the eigengroup elements $\mathcal{G}$ and the group orbifold elements $\hat{g}$ satisfy

$$
\begin{gathered}
g(T, \xi)=e^{i \beta^{a}(\xi) T_{a},} \quad \beta^{a}(\xi)^{\prime}=\beta^{b}(\xi)\left(\omega^{\dagger}\right)_{b}{ }^{a}=\beta^{b}(\xi) \omega_{b}{ }^{a} \\
g(T, \xi)^{\prime}=W(T) g(T, \xi) W^{\dagger}(T)=e^{i \beta^{a}(\xi)^{\prime} T_{a}}=e^{i \beta^{a}(\xi) T_{a}{ }^{\prime}} \\
\mathcal{G}(\mathcal{T}, \xi)=U(T) g(T, \xi) U^{\dagger}(T)=e^{i\left(\beta^{0 A}(\xi) \mathcal{T}_{0 A}(T)+\beta^{1 I}(\xi) \mathcal{T}_{1 I}(T)\right)} \\
\beta^{0 A}(\xi) \equiv \beta^{A}(\xi), \quad \beta^{0 A}(\xi)^{\prime}=\beta^{0 A}(\xi) \\
\mathcal{G}(\mathcal{T}, \xi)^{\prime}=E(T) \mathcal{G}(\mathcal{T}, \xi) E(T)^{*} \\
\hat{g}(\mathcal{T}, \xi)=e^{i\left(\hat{\beta}^{0 A}(\xi) \mathcal{T}_{0 A}(T)+\hat{\beta}^{1 I}(\xi) \mathcal{T}_{1 I}(T)\right)}, \quad \hat{\beta}^{0 A}(\xi+2 \pi)=\beta^{I I}(\xi), \quad \hat{\beta}^{0 A}(\xi), \quad \hat{\beta}^{1 I}(\xi+2 \pi)=-\beta^{1 I}(\xi) \\
\hat{g}(\mathcal{T}, \xi+2 \pi)=E(T) \hat{g}(\mathcal{T}, \xi) E(T)^{*} .
\end{gathered}
$$

Here $\xi$ is the spatial coordinate on the cylinder, $W(T)$ is the action of charge conjugation in rep $T$, $\{U(T), E(T)\}$ is the eigendata of the extended $H$-eigenvalue problem (3.25) and $\beta^{\prime}, g^{\prime}, \mathcal{G}^{\prime}$ are the responses of the untwisted objects to charge conjugation. The $\mathcal{T}$-selection rules (3.26e) guarantee the consistency of these responses, as well as the consistency of the monodromies of $\beta$ and $\hat{g}$. 
As an example, Eqs. (3.10b), 3.27a $), 3.28$ ) and (3.48b give the monodromy of the group orbifold elements

$$
\begin{array}{cc}
\hat{g}\left(\mathcal{T}\left(T^{\mathrm{adj}}\right), \xi+2 \pi\right)_{A}{ }^{B}=\hat{g}\left(\mathcal{T}\left(T^{\mathrm{adj}}\right), \xi\right)_{A}{ }^{B}, & \hat{g}\left(\mathcal{T}\left(T^{\mathrm{adj}}\right), \xi+2 \pi\right)_{I}{ }^{J}=\hat{g}\left(\mathcal{T}\left(T^{\mathrm{adj}}\right), \xi\right)_{I}{ }^{J} \\
\hat{g}\left(\mathcal{T}\left(T^{\mathrm{adj}}\right), \xi+2 \pi\right)_{A}{ }^{I}=-\hat{g}\left(\mathcal{T}\left(T^{\mathrm{adj}}\right), \xi\right)_{A}{ }^{I}, & \hat{g}\left(\mathcal{T}\left(T^{\mathrm{adj}}\right), \xi+2 \pi\right)_{I}{ }^{A}=-\hat{g}\left(\mathcal{T}\left(T^{\mathrm{adj}}\right), \xi\right)_{I}{ }^{A} \\
A \in \mathfrak{s o}(n), & I \in \mathfrak{s u}(n) / \mathfrak{s o}(n)
\end{array}
$$

in the twisted adjoint representation $\mathcal{T}\left(T^{\mathrm{adj}}\right)$.

For general twisted complex representations, we may use Eqs. (3.32) and (3.33) to write out the results (3.46), (3.47) and (3.48) in terms of the complex untwisted representation matrices $T^{(\mathrm{c})}$ :

$$
\begin{gathered}
g(T, \xi)=\exp \left(i \beta^{a}(\xi)\left(\begin{array}{cc}
T_{a}^{(\mathrm{c})} & 0 \\
0 & T_{a}^{(\mathrm{c})},
\end{array}\right)\right), \quad g(T, \xi)^{\prime}=\exp \left(i \beta^{a}(\xi)\left(\begin{array}{cc}
T_{a}^{(\mathrm{c}) \prime} & 0 \\
0 & T_{a}^{(\mathrm{c})}
\end{array}\right)\right) \\
\mathcal{G}(\mathcal{T}, \xi)=\exp \left(i\left(\begin{array}{cc}
\beta^{0 A}(\xi) T_{A}^{(\mathrm{c})} & \beta^{1 I}(\xi) T_{I}^{(\mathrm{c})} \\
\beta^{1 I}(\xi) T_{I}^{(\mathrm{c})} & \beta^{0 A}(\xi) T_{A}^{(\mathrm{c})}
\end{array}\right)\right) \\
\hat{g}(\mathcal{T}, \xi)=\exp \left(i\left(\begin{array}{cc}
\hat{\beta}^{0 A}(\xi) T_{A}^{(\mathrm{c})} & \hat{\beta}^{1 I}(\xi) T_{I}^{(\mathrm{c})} \\
\hat{\beta}^{1 I}(\xi) T_{I}^{(\mathrm{c})} & \hat{\beta}^{0 A}(\xi) T_{A}^{(\mathrm{c})}
\end{array}\right)\right) \\
\hat{g}(\mathcal{T}, \xi+2 \pi)=\exp \left(i\left(\begin{array}{cc}
\hat{\beta}^{0 A}(\xi) T_{A}^{(\mathrm{c})} & -\hat{\beta}^{1 I}(\xi) T_{I}^{(\mathrm{c})} \\
-\hat{\beta}^{1 I}(\xi) T_{I}^{(\mathrm{c})} & \hat{\beta}^{0 A}(\xi) T_{A}^{(\mathrm{c})}
\end{array}\right)\right) \\
=E(T) \hat{g}(\mathcal{T}, \xi) E(T)^{*},
\end{gathered}
$$

The consistency of the forms in (3.50d) and (3.500) is easily checked.

We turn next to the action formulation of the charge conjugation orbifolds. The untwisted charge-conjugation-invariant WZW action for any representation $T$ of $\mathfrak{s u}(n)$ can be taken as

$$
\begin{gathered}
S_{\mathrm{WZW}}[g(T)]=-\frac{k}{\epsilon y(T)}\left(\frac{1}{8 \pi} \int d^{2} \xi \operatorname{Tr}\left(g^{-1}(T) \partial_{+} g(T) g^{-1}(T) \partial_{-} g(T)\right)\right. \\
\left.+\frac{1}{12 \pi} \int_{\Gamma} \operatorname{Tr}\left(\left(g^{-1}(T) d g(T)\right)^{3}\right)\right) \\
\operatorname{Tr}\left(T_{a} T_{b}\right)=y(T) \delta_{a b}, \quad \epsilon= \begin{cases}1 & \text { for real reps } T \\
2 & \text { for complex reps } T^{(\mathrm{c})}\end{cases}
\end{gathered}
$$

$$
S_{\mathrm{WZW}}\left[g(T)^{\prime}\right]=S_{\mathrm{WZW}}[g(T)], \quad g(T, \xi)^{\prime}=W(T) g(T, \xi) W^{\dagger}(T) .
$$


For complex representations $T^{(\mathrm{c})}$, the block-diagonal group element 3.50a and the fact that $y\left(T^{(\mathrm{c}) \prime}\right)=y\left(\bar{T}^{(\mathrm{c})}\right)=y\left(T^{(\mathrm{c})}\right)$ tell us that the action $(\overline{3.51 \mathrm{a}})$ is in fact one half the sum of the WZW actions for $T^{(\mathrm{c})}$ and for $\bar{T}^{(\mathrm{c})} \simeq T^{(\mathrm{c}) \prime}$. Moreover, since the WZW action is numerically independent of $T$, the choice (3.51a) is in fact equal to the WZW action for either $T^{(\mathrm{c})}$ or $\bar{T}^{(\mathrm{c})}$.

Then following Ref. [10], one finds the action for any twisted representation $\mathcal{T}$ in twisted sector $\sigma=1$ of the charge conjugation orbifold on $\mathfrak{s u}(n)$

$$
\begin{gathered}
\hat{S}[\hat{g}(\mathcal{T})]=-\frac{k}{\epsilon y(T)}\left(\frac{1}{8 \pi} \int d^{2} \xi \operatorname{Tr}\left(\hat{g}^{-1}(\mathcal{T}, \sigma) \partial_{+} \hat{g}(\mathcal{T}, \sigma) \hat{g}^{-1}(\mathcal{T}, \sigma) \partial_{-} \hat{g}(\mathcal{T}, \sigma)\right)\right. \\
\left.+\frac{1}{12 \pi} \int_{\Gamma} \operatorname{Tr}\left(\left(\hat{g}^{-1}(\mathcal{T}, \sigma) d \hat{g}(\mathcal{T}, \sigma)\right)^{3}\right)\right) \\
\hat{S}[\hat{g}(\mathcal{T}, \xi+2 \pi)]=\hat{S}[\hat{g}(\mathcal{T}, \xi)], \quad \hat{g}(\mathcal{T}, \xi+2 \pi)=E(T) \hat{g}(\mathcal{T}, \xi) E(T)^{*} .
\end{gathered}
$$

The equations of motion of this action give twisted left- and right-mover classical matrix currents proportional to $\hat{g}^{-1} \partial_{+} \hat{g}$ and $\hat{g} \partial_{-} \hat{g}^{-1}$ whose monodromies agree with the monodromies of the quantum currents above.

Two further remarks about the case of complex representations are relevant here. We note first that the block-diagonal group elements $g$ in (3.50a and the matrix

$$
\left(\begin{array}{ll}
0 & 1 \\
1 & 0
\end{array}\right)=-i W(T)
$$

generate a non-connected Lie group [21]. Second, we emphasize that the corresponding group orbifold elements $\hat{g}$ are also reducible. To see this we consider the so-called group orbifold elements with twisted boundary conditions $\hat{\mathcal{G}}(T, \xi)$

$$
\begin{gathered}
\hat{g}(\mathcal{T}, \xi)=U(T) \hat{\mathcal{G}}(T, \xi) U^{\dagger}(T) \\
\hat{\mathcal{G}}(T, \xi)=\left(\begin{array}{cc}
\hat{\mathcal{G}}_{+}(T, \xi) & 0 \\
0 & \hat{\mathcal{G}}_{-}(T, \xi)
\end{array}\right), \quad \hat{\mathcal{G}}_{ \pm}(T, \xi)=\exp \left(i\left(\hat{\beta}^{0 A}(\xi) T_{A}^{(\mathrm{c})} \pm \hat{\beta}^{1 I}(\xi) T_{I}^{(\mathrm{c})}\right)\right) \\
\hat{\mathcal{G}}(T, \xi+2 \pi)=W(T) \hat{\mathcal{G}}(T, \xi) W^{\dagger}(T) \quad \leftrightarrow \quad \hat{\mathcal{G}}_{ \pm}(T, \xi+2 \pi)=\hat{\mathcal{G}}_{\mp}(T, \xi)
\end{gathered}
$$

which are block-diagonal with mixed monodromy: As seen in (3.54a), the group orbifold element $\hat{g}$ is the monodromy decomposition of $\hat{\mathcal{G}}$. Then the orbifold action (3.52a) decomposes into the sum of two terms $\hat{s}\left(\hat{\mathcal{G}}_{+}\right)+\hat{s}\left(\hat{\mathcal{G}}_{-}\right)$which mix under monodromy transformations.

\subsection{Correlator examples and undetermined parameters}

In this subsection we consider some correlator examples, pointing out and interpreting the existence of certain undetermined parameters in the solutions of the twisted KZ systems (3.41), (3.45) for complex representations.

We begin with the solution of (3.41) for the twisted left-mover one-point correlators

$$
\left\langle 0\left|\hat{g}_{+}(\mathcal{T}, z)\right| 0\right\rangle=C_{+}(\mathcal{T}) z^{-\frac{1}{2 k+Q_{g}} \sum_{I} \mathcal{T}_{1 I}(T) \mathcal{T}_{1 I}(T)}, \quad C_{+}(\mathcal{T}) \mathcal{T}_{0 A}(T)=0, \quad \forall A \in \mathfrak{s o}(n)
$$


We have checked for real and complex representations that the only solution for non-trivial $\mathcal{T}$ is

$$
\left\langle 0\left|\hat{g}_{+}(\mathcal{T}, z)\right| 0\right\rangle=0
$$

because there is no non-trivial solution to the global Ward identity in this case. This makes sense because our scalar twist-field state $|0\rangle$ is an $\mathfrak{s o}(n)$-singlet, and the same result $\left\langle\hat{g}_{-}(\mathcal{T}, \bar{z})\right\rangle=0$ is found for the twisted right movers when $\mathcal{T}$ is non-trivial.

We have also solved the twisted left-mover $\mathrm{KZ}$ equations for the two-point correlator in the case when both representations $T^{(\mathrm{c})(1)} \equiv T^{(1)}, T^{(\mathrm{c})(2)} \equiv T^{(2)}$ are taken as the fundamental representation of $\mathfrak{s u}(n)$. The result is

$$
\begin{gathered}
\left\langle 0\left|\hat{g}_{+}\left(\mathcal{T}^{(1)}, z_{1}\right) \hat{g}_{+}\left(\mathcal{T}^{(2)}, z_{2}\right)\right| 0\right\rangle=C_{+}(\mathcal{T})\left(z_{1} z_{2}\right)^{-\Delta_{n}}\left(\frac{z_{1} z_{2}}{z_{12}^{2}}\right)^{\alpha_{n}}\left(\frac{\sqrt{z_{1}}-\sqrt{z_{2}}}{\sqrt{z_{1}}+\sqrt{z_{2}}}\right)^{M(1,2)} \\
C_{+}(\mathcal{T})\left(\begin{array}{cc}
T_{A}^{(1)}+T_{A}^{(2)} & 0 \\
0 & T_{A}^{(1)}+T_{A}^{(2)}
\end{array}\right)=0, \quad \forall A \in \mathfrak{s o}(n) \\
\Delta_{n} \equiv \frac{n^{2}-1}{2 n(x+n)}, \quad \alpha_{n} \equiv \frac{n-1}{4(x+n)} \\
M(1,2) \equiv \frac{2}{2 k+Q_{g}} \sum_{I}\left(\begin{array}{cc}
0 & T_{I}^{(2)} \\
T_{I}^{(2)} & 0
\end{array}\right) \otimes\left(\begin{array}{cc}
0 & T_{I}^{(1)} \\
T_{I}^{(1)} & 0
\end{array}\right)
\end{gathered}
$$

where the quantity $\Delta_{n}$ is the conformal weight of the fundamental representation in the symmetric theory. To obtain this result, we used the explicit form (3.33) of the twisted representation matrices and the identities

$$
\begin{gathered}
\frac{1}{2 k+Q_{g}} \sum_{A} \mathcal{T}_{0 A}(T) \mathcal{T}_{0 A}(T)=\frac{1}{2 k+Q_{g}} \mathbb{1} \sum_{A} T_{A} T_{A}=\alpha_{n} \mathbb{1} \otimes \mathbb{1}_{n}, \quad \mathbb{1}=\left(\begin{array}{ll}
1 & 0 \\
0 & 1
\end{array}\right) \\
\frac{1}{2 k+Q_{g}} \sum_{I} \mathcal{T}_{1 I}(T) \mathcal{T}_{1 I}(T)=\frac{1}{2 k+Q_{g}} \mathbb{1} \sum_{I} T_{I} T_{I}=\left(\Delta_{n}-\alpha_{n}\right) \mathbb{1} \otimes \mathbb{1}_{n} \\
{\left[M(1,2), \mathcal{T}_{0 A}^{(1)}+\mathcal{T}_{0 A}^{(2)}\right]=0, \quad \forall A \in \mathfrak{s o}(n)}
\end{gathered}
$$

where 1 is the $2 \times 2$ unit matrix in the doubled space and $1_{n}$ is the $n \times n$ unit matrix in the space of the generalized Gell-Mann matrices.

We have further solved (3.45) for the twisted right-mover two-point correlator in this case, solved the full set of global Ward identities

$$
\begin{gathered}
C(\mathcal{T})=C_{-}(\mathcal{T}) C_{+}(\mathcal{T}) \\
C(\mathcal{T})\left(\begin{array}{cc}
T_{A}^{(1)}+T_{A}^{(2)} & 0 \\
0 & T_{A}^{(1)}+T_{A}^{(2)}
\end{array}\right)=\left(\begin{array}{cc}
T_{A}^{(1)}+T_{A}^{(2)} & 0 \\
0 & T_{A}^{(1)}+T_{A}^{(2)}
\end{array}\right) C(\mathcal{T})=0, \forall A \in \mathfrak{s o}(n)
\end{gathered}
$$


and combined these results with (3.57) to obtain the non-chiral two-point correlator for the full twisted vertex operators $\hat{g}=\hat{g}_{-} \hat{g}_{+}$. The final result is

$$
\begin{gathered}
\left\langle 0\left|\hat{g}\left(\mathcal{T}^{(1)}, \bar{z}_{1}, z_{1}\right) \hat{g}\left(\mathcal{T}^{(2)}, \bar{z}_{2}, z_{2}\right)\right| 0\right\rangle=C(\mathcal{T})\left|z_{1} z_{2}\right|^{-2 \Delta_{n}}\left|\frac{z_{1} z_{2}}{z_{12}^{2}}\right|^{2 \alpha_{n}}\left|\frac{\sqrt{z_{1}}-\sqrt{z_{2}}}{\sqrt{z_{1}}+\sqrt{z_{2}}}\right|^{2 M(1,2)} \\
C(\mathcal{T})=\left(\begin{array}{cc}
\gamma_{1} D & \gamma_{2} D \\
\gamma_{3} D & \gamma_{4} D
\end{array}\right), \quad[C(\mathcal{T}), M(1,2)]=0 \\
\left(T_{a}^{(1)}\right)_{\alpha_{1}}{ }^{\beta_{1}},\left(T_{a}^{(2)}\right)_{\alpha_{2}}{ }^{\beta_{2}} \quad: \quad D_{\beta_{1} \beta_{2}}{ }^{\alpha_{1} \alpha_{2}}=\delta_{\beta_{1} \beta_{2}} \delta^{\alpha_{1} \alpha_{2}}
\end{gathered}
$$

where $\gamma_{1} \ldots \gamma_{4}$ are arbitrary constants found in the general solution of the global Ward identities (3.59b).

Further determination of these constants is beyond the scope of this paper. However, we note here that the constants can be interpreted as undetermined couplings among the blocks of the quantum analogue $\hat{\mathcal{G}}(T, \bar{z}, z)$ of the group orbifold elements with twisted boundary conditions:

$$
\begin{gathered}
\hat{\mathcal{G}}(T, \bar{z}, z) \equiv U(T) \hat{g}(\mathcal{T}, \bar{z}, z) U^{\dagger}(T), \quad\left[U\left(T^{(1)}\right) \otimes U\left(T^{(2)}\right), C(\mathcal{T})\right]=0 \\
\left\langle 0\left|\hat{\mathcal{G}}\left(T^{(1)}, \bar{z}_{1}, z_{1}\right) \hat{\mathcal{G}}\left(T^{(2)}, \bar{z}_{2}, z_{2}\right)\right| 0\right\rangle=\left(\begin{array}{cc}
\gamma_{1} D & \gamma_{2} D \\
\gamma_{3} D & \gamma_{4} D
\end{array}\right)\left|z_{1} z_{2}\right|^{-2 \Delta_{n}}\left|\frac{z_{1} z_{2}}{z_{12}^{2}}\right|^{2 \alpha_{n}}\left|\frac{\sqrt{z_{1}}-\sqrt{z_{2}}}{\sqrt{z_{1}}+\sqrt{z_{2}}}\right|^{2 N(1,2)} \\
N(1,2) \equiv \frac{2}{2 k+Q_{g}} \sum_{I}\left(\begin{array}{cc}
T_{I}^{(2)} & 0 \\
0 & -T_{I}^{(2)}
\end{array}\right) \otimes\left(\begin{array}{cc}
T_{I}^{(1)} & 0 \\
0 & -T_{I}^{(1)}
\end{array}\right)
\end{gathered}
$$

This suggests that a natural boundary condition is $\gamma_{2}=\gamma_{3}=0$, i.e. a block diagonal solution for the $\hat{\mathcal{G}}$ correlator. Such a boundary condition could in principle be implemented in general because, for any number of $\hat{\mathcal{G}}$ 's in any set of complex representations, we find the block-diagonal twisted KZ system:

$$
\begin{aligned}
& \hat{\mathcal{A}}(T, \bar{z}, z) \equiv\left\langle 0\left|\hat{\mathcal{G}}\left(T^{(1)}, \bar{z}_{1}, z_{1}\right) \cdots \hat{\mathcal{G}}\left(T^{(N)}, \bar{z}_{N}, z_{N}\right)\right| 0\right\rangle \\
& \partial_{\mu} \hat{\mathcal{A}}(T, \bar{z}, z)=\hat{\mathcal{A}}(T, \bar{z}, z) \hat{\mathcal{W}}_{\mu}(T, z), \quad \bar{\partial}_{\mu} \hat{\mathcal{A}}(T, \bar{z}, z)=\hat{\mathcal{W}}_{\mu}(T, \bar{z}) \hat{\mathcal{A}}(T, \bar{z}, z) \\
& \hat{\mathcal{W}}_{\mu}(T, z)=\left.\hat{W}_{\mu}(\mathcal{T}, z)\right|_{\mathcal{T} \rightarrow T}, \quad \hat{\mathcal{W}}_{\mu}(T, \bar{z})=\left.\hat{\bar{W}}_{\mu}(\mathcal{T}, \bar{z})\right|_{\mathcal{T} \rightarrow T} \\
& \hat{\mathcal{A}}(T, \bar{z}, z)\left(\sum_{\mu=1}^{N} T_{A}^{(\mu)}\right)=\left(\sum_{\mu=1}^{N} T_{A}^{(\mu)}\right) \hat{\mathcal{A}}(T, \bar{z}, z)=0, \quad \forall A \in \mathfrak{s o}(n) .
\end{aligned}
$$

Here the twisted connections $\hat{W}$ and $\hat{\bar{W}}$ are given in (3.39), (3.45) and the untwisted representation matrices $T$ have the block-diagonal form in 3.30a). 


\section{Acknowledgements}

For helpful discussions we thank J. de Boer, C. Helfgott, J. Lepowsky, C. Schweigert, F. Wagner and N. Warner. MBH and NO thank the Niels Bohr Institute for hospitality and support. The work of $\mathrm{MBH}$ was supported in part by the Director, Office of Energy Research, Office of High Energy and Nuclear Physics, Division of High Energy Physics of the U.S. Department of Energy under Contract DE-AC03-76SF00098 and in part by the National Science Foundation under grant PHY95-14797. The work of NO is supported in part by the stichting FOM and the European Commission RTN programme HPRN-CT-2000-00131.

\section{A The $H$-eigenvalue problem for permutation groups}

In this appendix we use the modified notation of Subsec. 2.6 to solve the $H$-eigenvalue problem [3, 4, 10] for all permutation groups $H \subset S_{N}$. The solution of the $H$-eigenvalue problem also gives an explicit derivation of various results which were argued more abstractly for the WZW permutation orbifolds in Ref. [10].

We begin with the permutation-invariant system

$$
\begin{gathered}
\oplus_{I=0}^{K-1} \mathfrak{g}^{I}, \quad \mathfrak{g}^{I} \simeq \mathfrak{g}, \quad K \leq N \\
J_{a I}(z) J_{b J}(w)=\delta_{I J}\left\{\frac{k \eta_{a b}}{(z-w)^{2}}+\frac{i f_{a b}^{c} J_{c I}(w)}{z-w}\right\}+\mathcal{O}(z-w)^{0} \\
T(z)=\frac{1}{2 k+Q_{\mathfrak{g}}} \sum_{I=0}^{K-1} \eta^{a b}: J_{a I}(z) J_{b I}(z):, \quad a, b=1 \ldots \operatorname{dim} \mathfrak{g}
\end{gathered}
$$

where the automorphism group acts on the currents and stress tensor as $J_{a I}{ }^{\prime}=\omega\left(h_{\sigma}\right)_{I}^{J} J_{a J}$, $h_{\sigma} \in H$ (permutation) $\subset S_{N}$ and $T(z)^{\prime}=T(z)$. The eigencurrents [3, 1, 10]

$$
\begin{gathered}
\mathcal{J}_{n(r) a j}(z)=\chi_{n(r) a j}(\sigma) U(\sigma)_{n(r) j}{ }^{I} J_{a I}(z) \\
\mathcal{J}_{n(r) a j}(z)^{\prime}=E_{n(r)}(\sigma) \mathcal{J}_{n(r) a j}(z), \quad E_{n(r)}(\sigma)=e^{-2 \pi i \frac{n(r)}{\rho(\sigma)}}
\end{gathered}
$$

have diagonal response under $H$, where $\omega\left(h_{\sigma}\right) U^{\dagger}(\sigma)=U^{\dagger}(\sigma) E(\sigma), \sigma=0, \ldots, N_{c}-1$ is the $H$-eigenvalue problem [3, 4, 10] and the $\chi$ 's are normalization constants.

To study the $H$-eigenvalue problem most efficiently, it is convenient to introduce a new basis. For each $h_{\sigma} \in H$ (permutation), we may relabel the copies $I$ to obtain a $\sigma$-dependent cycle basis of the type constructed for $H=S_{N}$ in Ref. [4]

$$
\begin{gathered}
I \rightarrow \hat{j} j, \quad J_{a I} \rightarrow \tilde{J}_{a \hat{j} j} \\
\tilde{J}_{a \hat{j} j}(z) \tilde{J}_{b \hat{l} l}(w)=\delta_{j l} \delta_{\hat{j}-\hat{l}, 0 \bmod f_{j}(\sigma)}\left\{\frac{k \eta_{a b}}{(z-w)^{2}}+\frac{i f_{a b}{ }^{c} \tilde{J}_{c \hat{j} j}(w)}{z-w}\right\}+\mathcal{O}(z-w)^{0}
\end{gathered}
$$




$$
\begin{gathered}
T(z)=\frac{1}{2 k+Q_{\mathfrak{g}}} \sum_{j} \sum_{\hat{j}=0}^{f_{j}(\sigma)-1} \eta^{a b}: \tilde{J}_{a \hat{j} j}(z) \tilde{J}_{b \hat{j} j}(z): \\
\mathcal{J}_{n(r) a j}(z)=\chi_{n(r) a j}(\sigma) U(\sigma)_{n(r) a j} \hat{l} l \tilde{J}_{a \hat{l} l}(z) \\
\overline{\hat{j}}=0, \ldots, f_{j}(\sigma)-1, \quad \overline{\hat{l}}=0, \ldots, f_{l}(\sigma)-1, \quad \sum_{j} f_{j}(\sigma)=K .
\end{gathered}
$$

Here the unhatted indices $j$ or $l$ label the disjoint cycles (of size and order $f_{j}(\sigma)$ or $f_{l}(\sigma)$ ) of each $h_{\sigma} \in H$ and the hatted indices $\hat{j}$ or $\hat{l}$ run inside the disjoint cycle $j$ or $l$. The labelling inside each disjoint cycle is periodic $\hat{j} \rightarrow \hat{j} \pm f_{j}(\sigma)$ and the barred quantities $\overline{\hat{j}}, \hat{i}$ in (A.3e are the pullbacks of $\hat{j}$ and $\hat{l}$ into the fundamental range. The cycle basis can be chosen so that each $h_{\sigma}$ has matrix representation

$$
\omega\left(h_{\sigma}\right)_{a \hat{j j}}^{b \hat{l} l}=\delta_{a}^{b} \omega\left(h_{\sigma}\right)_{\hat{j} j}^{\hat{l} l}, \quad \omega\left(h_{\sigma}\right)_{\hat{j} j}^{\hat{l} l}=\delta_{j}^{l} \delta_{\hat{j}+1, \hat{l} \bmod f_{j}(\sigma)}, \quad \sigma=0, \ldots, N_{c}-1
$$

that is so that the automorphism is a cyclic permutation $\hat{j} \rightarrow \hat{j}+1$ in each disjoint cycle.

Examples.

a) The cycle basis is discussed explicitly for the permutation groups $S_{N}$ in Ref. [4], with

$$
K=N, \quad f_{j}(\sigma)=\sigma_{j}, \quad \sigma_{j+1} \leq \sigma_{j}, \quad j=0, \ldots, n(\vec{\sigma})-1, \quad \sum_{j=0}^{n(\vec{\sigma})-1} \sigma_{j}=N
$$

This choice gives one element $h_{\sigma} \in S_{N}$ in each conjugacy class of $S_{N}$.

b) For the cyclic permutation groups $\mathbb{Z}_{\lambda}$ one has

$$
K=\lambda, \quad f_{j}(\sigma)=\rho(\sigma), \quad \overline{\hat{j}}=0, \ldots, \rho(\sigma)-1, \quad j=0, \ldots, \frac{\lambda}{\rho(\sigma)}-1
$$

where $\rho(\sigma)$ is the order of $h_{\sigma} \in \mathbb{Z}_{\lambda}$ and $\sigma=0, \ldots, \lambda-1$. In this case we give some simple examples of the relabelling $I \rightarrow \hat{j} j$ : For $\lambda=$ prime, one has $\rho(\sigma)=\lambda, \sigma=1, \ldots, \lambda-1$ and a single disjoint cycle $j=0$. Choosing also $\sigma=2$ we maintain a shift $\hat{j} \rightarrow \hat{j}+1$ in the single disjoint cycle by relabelling

$$
\begin{gathered}
\hat{j}=(0,1, \ldots, \lambda-1) \quad \leftrightarrow I=(0,2, \ldots, \lambda-1,1,3, \ldots, \lambda-2) \\
\tilde{J}_{a \hat{j}}=\tilde{J}_{a \hat{j} 0}=\left(J_{a 0}, J_{a 2}, \ldots, J_{a, \lambda-1}, J_{a 1}, J_{a 3}, \ldots, J_{a, \lambda-2}\right) .
\end{gathered}
$$

For $\lambda=4, \sigma=2$ we have $\rho(2)=2$ and two disjoint cycles $j=0$ and 1 , and we may relabel as follows:

$$
\tilde{J}_{a \hat{j} 0}=\left(J_{a 0}, J_{a 2}\right), \quad \tilde{J}_{a \hat{j} 1}=\left(J_{a 1}, J_{a 3}\right)
$$

where $\hat{j}=0$ and 1 in each disjoint cycle. 
We consider now the $H$-eigenvalue problem and its solution in the cycle basis:

$$
\begin{aligned}
& \sum_{l} \sum_{\hat{l}=0}^{f_{l}(\sigma)-1} \omega\left(h_{\sigma}\right)_{\hat{j} j}^{\hat{l} l} U^{\dagger}(\sigma)_{\hat{l} l}^{\hat{m} m}=U^{\dagger}(\sigma)_{\hat{j} j}^{\hat{m} m} E_{\hat{m}}^{m}(\sigma) \\
& U^{\dagger}(\sigma)_{\hat{j} j}^{\hat{l} l}=\frac{\delta_{j}^{l}}{\sqrt{f_{j}(\sigma)}} e^{-2 \pi i \frac{\hat{j} \hat{l}}{f_{j}(\sigma)}}, \quad U(\sigma)_{\hat{j} j}^{\hat{l} l}=\frac{\delta_{j}^{l}}{\sqrt{f_{j}(\sigma)}} e^{2 \pi i \frac{\hat{j} \hat{l}}{f_{j}(\sigma)}}, \quad E_{\hat{j}}^{j}=e^{-2 \pi i \frac{\hat{j}}{f_{j}(\sigma)}} \\
& \sum_{l} \sum_{\hat{l}=0}^{f_{j}(\sigma)-1} U^{\dagger}(\sigma)_{\hat{j} j}^{\hat{l} l} U(\sigma)_{\hat{l} l}^{\hat{m} m}=\delta_{j}^{m} \delta_{\hat{j}-\hat{m}, 0 \bmod f_{j}(\sigma)} \\
& \overline{\hat{j}}=0, \ldots, f_{j}(\sigma)-1, \quad \overline{\hat{l}}=0, \ldots, f_{l}(\sigma)-1, \quad \overline{\hat{m}}=0, \ldots, f_{m}(\sigma)-1 .
\end{aligned}
$$

Here the relabelling of the spectral integers

$$
n(r) j \rightarrow \hat{j j}, \quad U^{\dagger}(\sigma)_{n(r) j}{ }^{\hat{l} l} \rightarrow U^{\dagger}(\sigma)_{\hat{j} j}^{\hat{l} l}
$$

is dictated by the form of the eigenvalue problem, and the correspondence

$$
\frac{n(r)}{\rho(\sigma)}=\frac{\hat{j}}{f_{j}(\sigma)}, \quad \overline{\hat{j}}=0, \ldots, f_{j}(\sigma)-1
$$

is obtained by comparing the eigenvalues $E_{\hat{j}}^{j}$ to the standard form of the eigenvalues in (A.2). In what follows we use the correspondence (A.11) to relabel all the spectral integers $n(r) \rightarrow \hat{j}$.

Returning to the eigencurrents $\mathcal{J}_{n(r) a j} \rightarrow \mathcal{J}_{\hat{j} a j}$ we choose the normalization

$$
\chi_{n(r) a j}(\sigma) \rightarrow \chi_{\hat{j a j}}(\sigma)=\sqrt{f_{j}(\sigma)} .
$$

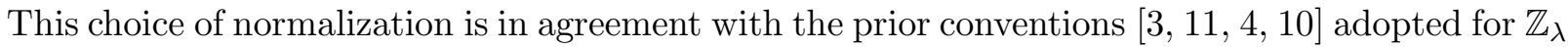
and $S_{N}$. Then, combining (A.3d), (A.9) and (A.12) we find an explicit form for the eigencurrents

$$
\begin{aligned}
& \mathcal{J}_{\hat{j} a j}(z)=\sqrt{f_{j}(\sigma)} U(\sigma)_{\hat{j} j}^{\hat{l} l} \tilde{J}_{a \hat{l} l}(z)=\sum_{\hat{j}^{\prime}=0}^{f_{j}(\sigma)-1} e^{2 \pi i \frac{\hat{j} \hat{j}^{\prime}}{f_{j}(\sigma)}} \tilde{J}_{a \hat{j}^{\prime} j}(z), \quad \mathcal{J}_{\hat{j} a j}(z)^{\prime}=e^{-2 \pi i \frac{\hat{j}}{f_{j}(\sigma)}} \mathcal{J}_{\hat{j} a j}(z) \\
& \tilde{J}_{a \hat{j} j}(z)=\frac{1}{f_{j}(\sigma)} \sum_{\hat{j}^{\prime}=0}^{f_{j}(\sigma)-1} e^{-2 \pi i \frac{\hat{j} \hat{j}^{\prime}}{f_{j}(\sigma)}} \mathcal{J}_{a \hat{j}^{\prime} j}(z), \quad \sum_{j} \mathcal{J}_{0 a j}(z)=\sum_{j} \sum_{\hat{j}=0}^{f_{j}(\sigma)-1} \tilde{J}_{a \hat{j} j}(z)=\sum_{I=0}^{K-1} J_{a I}(z)
\end{aligned}
$$

and the OPEs of the eigencurrents are

$$
\mathcal{J}_{\hat{j} a j}(z) \mathcal{J}_{\hat{l} b l}(w)=\frac{\mathcal{G}_{\hat{j} a j ; \hat{l} b l}(\sigma)}{(z-w)^{2}}+i \mathcal{F}_{\hat{j} a j ; \hat{l} b l}{ }^{\hat{j}+\hat{l}, c m}(\sigma) \frac{\mathcal{J}_{\hat{j}+\hat{l}, c m}(w)}{z-w}+\mathcal{O}(z-w)^{0}
$$




$$
\begin{gathered}
\mathcal{G}_{\hat{j} a j ; \hat{l} b l}(\sigma)=\eta_{a b} \hat{k}_{j}(\sigma) \delta_{j l} \delta_{\hat{j}+\hat{l}, 0 \bmod f_{j}(\sigma)}, \quad \hat{k}_{j}(\sigma)=k f_{j}(\sigma) \\
\mathcal{F}_{\hat{j} a j ; \hat{l} b l}{ }^{\hat{m} c m}(\sigma)=f_{a b}{ }^{c} \delta_{j l} \delta_{l}^{m} \delta_{\hat{j}+\hat{l}-\hat{m}, 0 \bmod f_{j}(\sigma)}
\end{gathered}
$$

We may also rewrite the stress tensor in terms of the eigencurrents

$$
\begin{gathered}
\mathcal{L}_{\hat{\mathfrak{g}}(\sigma)}^{\hat{j} a j ; \hat{l} b l}(\sigma)=\frac{k}{2 k+Q_{\mathfrak{g}}} \mathcal{G}^{\hat{j} a j ; \hat{l} b l}(\sigma), \quad \mathcal{G}^{\hat{j} a j ; \hat{l} b l}(\sigma)=\eta^{a b} \hat{k}_{j}^{-1}(\sigma) \delta^{j l} \delta_{\hat{j}+\hat{l}, 0 \bmod f_{j}(\sigma)} \\
T(z)=\sum_{j} \mathcal{L}_{\hat{\mathfrak{g}}(\sigma)}^{\hat{j} a j ; \hat{l} b l}(\sigma): \mathcal{J}_{\hat{j} a j}(z) \mathcal{J}_{\hat{l} b l}(z):=\frac{1}{2 k+Q_{\mathfrak{g}}} \sum_{j} \frac{1}{f_{j}(\sigma)} \sum_{\hat{j}=0}^{f_{j}(\sigma)-1} \eta^{a b}: \mathcal{J}_{\hat{j} a j}(z) \mathcal{J}_{-\hat{j}, b j}(z):
\end{gathered}
$$

where : $:$ is operator product normal ordering [2, 3, 11, 4, 10]. Under the relabelling $n(r) \rightarrow \hat{j}$, these results for $\mathcal{G}, \mathcal{F}$ and $\mathcal{L}$ are equivalent to those given in Ref. [10].

Then using local isomorphisms $\mathcal{J} \underset{\sigma}{\longrightarrow} \hat{J}$ [3] we obtain for sector $\sigma$ of the general WZW permutation orbifold

$$
\begin{aligned}
& \hat{J}_{\hat{j} a j}(z) \hat{J}_{\hat{l} b l}(w)=\frac{\mathcal{G}_{\hat{j} a j ; \hat{l} b l}(\sigma)}{(z-w)^{2}}+i \mathcal{F}_{\hat{j} a j ; \hat{l} b l} \hat{j}^{\hat{j} \hat{l}, c m}(\sigma) \frac{\hat{J}_{\hat{j}+\hat{l}, c m}(w)}{z-w}+\mathcal{O}(z-w)^{0} \\
& \hat{J}_{\hat{j} a j}\left(z e^{2 \pi i}\right)=e^{-2 \pi i \frac{\hat{j}}{f_{j}(\sigma)}} \hat{J}_{\hat{j} a j}(z), \quad \hat{J}_{\hat{j} a j}(z)=\sum_{m \in \mathbb{Z}} \hat{J}_{\hat{j} a j}\left(m+\frac{\hat{j}}{f_{j}(\sigma)}\right) z^{-\left(m+\frac{\hat{j}}{f_{j}(\sigma)}\right)-1} \\
& {\left[\hat{J}_{\hat{j} a j}\left(m+\frac{\hat{j}}{f_{j}(\sigma)}\right), \hat{J}_{\hat{l} b l}\left(n+\frac{\hat{l}}{f_{j}(\sigma)}\right)\right]=\delta_{j l}\left(i f_{a b}{ }^{c} \hat{J}_{\hat{j}+\hat{l}}\left(m+n+\frac{\hat{j}+\hat{l}}{f_{j}(\sigma)}\right)\right.} \\
& \left.+\hat{k}_{j}(\sigma) \eta_{a b}\left(m+\frac{\hat{j}}{f_{j}(\sigma)}\right) \delta_{m+n+\frac{\hat{j}+\hat{l}}{f_{j}(\sigma)}, 0}\right) \\
& \hat{T}_{\sigma}(z)=\mathcal{L}_{\hat{\mathfrak{g}}(\sigma)}^{\hat{j} a j ; \hat{l} b l}(\sigma): \hat{J}_{\hat{j} a j}(z) \hat{J}_{\hat{l} b l}(z):=\frac{1}{2 k+Q_{\mathfrak{g}}} \sum_{j} \frac{1}{f_{j}(\sigma)} \sum_{\hat{j}=0}^{f_{j}(\sigma)-1} \eta^{a b}: \hat{J}_{\hat{j} a j}(z) \hat{J}_{-\hat{j} b j}(z):
\end{aligned}
$$

Under the relabelling $n(r) a j \rightarrow \hat{j} a j$, these results are also equivalent to those given in Ref. [10].

For the special case $H=\mathbb{Z}_{\lambda}$ another form of the eigencurrents

$$
\mathcal{J}_{\text {raj }}(z)=\sum_{s=0}^{\rho(\sigma)-1} e^{2 \pi i \frac{N(\sigma) r s}{\rho(\sigma)}} J_{a, \frac{\lambda}{\rho(\sigma)} s+j}(z), \quad \overline{\hat{j}}=\bar{r}=0, \ldots, \rho(\sigma)-1, \quad j=0, \ldots, \frac{\lambda}{\rho(\sigma)}-1
$$

was given in Eq. (3.28) of Ref. [3], where the integers $N(\sigma)$ are also defined. This is the form taken by the $\mathbb{Z}_{\lambda}$ eigencurrents when expressed in terms of the untwisted currents $J_{a I}$ of the original $I$ 
basis. The two expressions (A.17) and A.13a for $H=\mathbb{Z}_{\lambda}$ are equal because the eigencurrents are independent of the basis choice $I$ or $\hat{j} j$. To see how this works in a simple example, consider the case $\lambda=3, \sigma=2$ with $\rho(2)=3, N(2)=2$ and $j=0$ :

$$
\mathcal{J}_{r a 0}(z)=\sum_{s=0}^{2} e^{4 \pi i \frac{r s}{3}} J_{a s}=\sum_{s=0}^{2} e^{2 \pi i \frac{r s}{3}} \tilde{J}_{a s}, \quad \bar{r}=0,1,2, \quad \tilde{J}_{a s}=\left(J_{a 0}, J_{a 2}, J_{a 1}\right)
$$

The form of $\tilde{J}_{a s}$ in (A.18) is a special case of the relabelling (A.7b).

For the extended $H$-eigenvalue problem, we have the solution 10

$$
U^{\dagger}(T, \sigma)_{\alpha I}^{n(r) \beta j} \rightarrow U^{\dagger}(T, \sigma)_{\alpha \hat{j} j}^{\hat{l} \beta l}=\delta_{\alpha}^{\beta} U^{\dagger}(\sigma)_{\hat{j} j}^{\hat{l} l}, \quad \alpha, \beta=1 \ldots \operatorname{dim} T
$$

where $T$ is any irrep of $\mathfrak{g}$ and $U^{\dagger}(\sigma)$ is the solution (A.9) to the $H$-eigenvalue problem. Then we may evaluate Eq. (7.7e) of Ref. [10] to find the factorized form of the twisted representation matrices $\mathcal{T}$

$$
\begin{gathered}
\mathcal{T}_{n(r) j}(T, \sigma) \rightarrow \mathcal{T}_{\hat{j} a j}(T, \sigma), \quad\left[\mathcal{T}_{\hat{j} a j}(T, \sigma), \mathcal{T}_{\hat{l} b l}(T, \sigma)\right]=i \delta_{j l} f_{a b}{ }^{c} \mathcal{T}_{\hat{j}+\hat{l}, c j}(T, \sigma) \\
\mathcal{T}_{\hat{j} a j}(T, \sigma)=T_{a} t_{\hat{j} j}(\sigma), \quad\left[T_{a}, T_{b}\right]=i f_{a b}{ }^{c} T_{c}, \quad t_{\hat{j} j}(\sigma)_{\hat{l} l} \hat{m} m=\delta_{j l} \delta_{l}^{m} \delta_{\hat{j}+\hat{l}-\hat{m}, 0 \bmod f_{j}(\sigma)}
\end{gathered}
$$

in agreement with the result given in Ref. [10]. The formulae (2.48f $),(2.48 \mathrm{~g})$ and $(2.48 \mathrm{~h})$ are the abelian limit of (A.20), as expected.

\section{B More about the WZW permutation orbifolds}

We continue with the WZW permutation orbifolds, using the spectral index relabelling

$$
n(r) a j \quad \rightarrow \hat{j} a j, \quad N(r) \alpha j \rightarrow \hat{j} \alpha j, \quad a=1 \ldots \operatorname{dim} \mathfrak{g}, \quad \alpha=1 \ldots \operatorname{dim} T
$$

to rewrite more of the results of Ref. [10]. First we rewrite the results of Ref. [10] for the left- and right-mover ground state conformal weights in each sector $\sigma$ of all WZW permutation orbifolds 10

$$
\begin{gathered}
\hat{\Delta}_{0}(\sigma)=\hat{\bar{\Delta}}_{0}(\sigma)=\frac{c_{\mathfrak{g}}}{2} \sum_{r j} \frac{\bar{n}(r)}{2 \rho(\sigma)}\left(1-\frac{\bar{n}(r)}{\rho(\sigma)}\right)=\frac{c_{\mathfrak{g}}}{2} \sum_{j} \sum_{\hat{j}=1}^{f_{j}(\sigma)-1} \frac{\hat{j}}{2 f_{j}(\sigma)}\left(1-\frac{\hat{j}}{f_{j}(\sigma)}\right) \\
=\frac{c_{\mathfrak{g}}}{24} \sum_{j}\left(f_{j}(\sigma)-\frac{1}{f_{j}(\sigma)}\right)=\frac{c_{\mathfrak{g}}}{24}\left(K-\sum_{j} \frac{1}{f_{j}(\sigma)}\right) \\
c_{\mathfrak{g}}=\frac{x_{\mathfrak{g}} \operatorname{dim} \mathfrak{g}}{x_{\mathfrak{g}}+\tilde{h}_{\mathfrak{g}}}, \quad x_{\mathfrak{g}} \equiv \frac{2 k}{\psi_{\mathfrak{g}}^{2}}, \quad \tilde{h}_{\mathfrak{g}} \equiv \frac{Q_{\mathfrak{g}}}{\psi_{\mathfrak{g}}^{2}}
\end{gathered}
$$

where $K$ is defined in (A.1) and $c_{\mathfrak{g}}$ is the central charge of the affine-Sugawara construction [5, 6, 23, 24] on affine g. Eq. (2.49) is the abelian limit of (B.2), as expected. 
To go further it will be useful to have the identities

$$
\begin{aligned}
& \mathcal{L}_{\hat{\mathfrak{g}}(\sigma)}^{\hat{j} a j ;-\hat{j}, b l}(\sigma) \mathcal{T}_{\hat{j} a j} \mathcal{T}_{-\hat{j}, b l}=\Delta_{\mathfrak{g}}(T) \mathbb{1}, \quad \frac{\left(\eta^{a b} T_{a} T_{b}\right)_{\alpha}{ }^{\beta}}{2 k+Q_{\mathfrak{g}}}=\Delta_{\mathfrak{g}}(T) \delta_{\alpha}^{\beta} \\
& \mathcal{L}_{\hat{\mathfrak{g}}(\sigma)}^{0 a j ; 0 b l}(\sigma) \mathcal{T}_{0 a j} \mathcal{T}_{0 b l}=\Delta_{\mathfrak{g}}(T) \sum_{j} \frac{1}{f_{j}(\sigma)} t_{0 j}(\sigma) \\
& 2 \mathcal{L}_{\hat{\mathfrak{g}}(\sigma)}^{n(r) a j ;-n(r) b l}(\sigma) \frac{\bar{n}(r)}{\rho(\sigma)} \mathcal{T}_{n(r) a j} \mathcal{T}_{-n(r), b l}=\mathcal{L}_{\hat{\mathfrak{g}}(\sigma)}^{n(r) a j ;-n(r) b l}(\sigma) \mathcal{T}_{n(r) a j} \mathcal{T}_{-n(r), b l}\left(1-\delta_{\bar{n}(r), 0}\right) \\
& =\Delta_{\mathfrak{g}}(T)\left(\mathbb{1}-\sum_{j} \frac{1}{f_{j}(\sigma)} t_{0 j}(\sigma)\right)
\end{aligned}
$$

where we have used (A.15a $)$ and the factorized form $\mathcal{T}=T t$ of the twisted representation matrices. These results are analogous to those given for the abelian case in Subsec. 2.6.

Then the twisted left-mover KZ systems of the WZW permutation orbifolds take the form 10

$$
\begin{gathered}
\partial_{\mu} \hat{A}_{+}(\sigma)=\hat{A}_{+}(\sigma) \hat{W}_{\mu}(\sigma), \quad \hat{A}_{+}(\sigma) \equiv \hat{A}_{+}(\mathcal{T}, z, \sigma), \quad \sigma=0, \ldots, \rho(\sigma)-1 \\
\hat{W}_{\mu}(\sigma)=\frac{2}{2 k+Q_{\mathfrak{g}}} \sum_{\nu \neq \mu} \eta^{a b} \frac{T_{a}^{(\nu)} T_{b}^{(\mu)}}{z_{\mu \nu}} \sum_{j} \sum_{\hat{j}=0}^{f_{j}(\sigma)-1}\left(\frac{z_{\nu}}{z_{\mu}}\right)^{\frac{\hat{j}}{f_{j}(\sigma)}} \frac{t_{\hat{j} j}^{(\nu)}(\sigma) t_{-\hat{j}, j}^{(\mu)}(\sigma)}{f_{j}(\sigma)} \\
-\frac{\Delta_{\mathfrak{g}}\left(T^{(\mu)}\right)}{z_{\mu}}\left(\mathbb{1}-\sum_{j} \frac{1}{f_{j}(\sigma)} t_{0 j}^{(\mu)}(\sigma)\right) \\
\hat{A}_{+}(\sigma) \sum_{\mu=1}^{N} T_{a}^{(\mu)} t_{0 j}^{(\mu)}(\sigma)=0 .
\end{gathered}
$$

Finally we note the form of the general left-mover two-point correlators of Ref. 10] in this notation

$$
\begin{aligned}
& \hat{A}_{+}(1,2)=\left\langle\hat{g}_{+}\left(\mathcal{T}^{1}, z_{1}, \sigma\right) \hat{g}_{+}\left(\mathcal{T}^{2}, z_{2}, \sigma\right)\right\rangle_{\sigma} \\
& =C_{+}(\mathcal{T}, \sigma) z_{1}^{-\Delta_{\mathfrak{g}}\left(T^{(1)}\right)\left(\mathbb{1}-\sum_{j} \frac{1}{f_{j}(\sigma)} t_{0 j}^{(1)}(\sigma)\right)} z_{2}^{-\Delta_{\mathfrak{g}}\left(T^{(2)}\right)\left(\mathbb{1}-\sum_{j} \frac{1}{f_{j}(\sigma)} t_{0 j}^{(2)}(\sigma)\right)} z_{12}^{-2 \Delta_{\mathfrak{g}}\left(T^{(1)}\right) \sum_{j} \frac{1}{f_{j}(\sigma)} t_{0 j}^{(1)}(\sigma)} \\
& \times \exp \left(\frac{2}{2 k+Q_{\mathfrak{g}}} T_{a}^{(2)} \eta^{a b} T_{b}^{(1)} \sum_{j} \frac{1}{f_{j}(\sigma)} \sum_{\hat{j}=1}^{f_{j}(\sigma)-1} t_{\hat{j} j}^{(2)}(\sigma) t_{-\hat{j}, j}^{(1)}(\sigma) I_{\frac{\hat{j}}{f_{j}(\sigma)}}\left(\frac{z_{1}}{z_{2}}\right)\right) \\
& C_{+}(\mathcal{T}, \sigma)\left(T_{a}^{(1)} t_{0 j}^{(1)}(\sigma)+T_{a}^{(2)} t_{0 j}^{(2)}(\sigma)\right)=0
\end{aligned}
$$

where the indefinite integrals $I$ were evaluated in Ref. [10]. The abelian limit of (B.5) is in agreement with the result (2.55) of the text. 


\section{An identity for the full correlators of the abelian orbifolds}

In this appendix, we verify the symmetry $F(\kappa, \rho)=F(\rho, \kappa)$ stated in Eq. (2.97) of the text. The steps followed here parallel those used in Ref. [10] to prove $1 \leftrightarrow 2$ symmetry for the non-chiral two-point correlators of the WZW cyclic permutation orbifolds.

We start with the definition $(\rho \neq k)$

$$
\begin{aligned}
F(\rho, \kappa) \equiv & \mathcal{G}^{n(r) \mu ;-n(r), \nu}(\sigma)\left(1-\delta_{\bar{n}(r), 0}\right) \\
& \times\left(\mathcal{T}_{n(r) \mu}^{(\rho)} \mathcal{T}_{-n(r), \nu}^{(\kappa)} I_{\bar{n}(r)}\left(\frac{z_{\rho}}{z_{\kappa}}, \infty\right)+\mathcal{T}_{n(r) \mu}^{(\kappa)} \mathcal{T}_{-n(r), \nu}^{(\rho)} I_{\bar{n}(r)}\left(\frac{\bar{z}_{\rho}}{\bar{z}_{\kappa}}, \infty\right)\right)
\end{aligned}
$$

Then we compare this to $F(\kappa, \rho)$, following the steps

$$
\begin{aligned}
F(\kappa, \rho)= & \mathcal{G}^{n(r)-\rho(\sigma), \mu ; \rho(\sigma)-n(r), \nu}(\sigma)\left(1-\delta_{\bar{n}(r), 0}\right) \\
\times & \left(\mathcal{T}_{\rho(\sigma)-n(r), \nu}^{(\rho)} \mathcal{T}_{n(r)-\rho(\sigma), \mu}^{(\kappa)} \frac{\rho(\sigma)-\bar{n}(r)}{\rho(\sigma)}\left(\frac{z_{\rho}}{z_{\kappa}}, 0\right)+\mathcal{T}_{\rho(\sigma)-n(r), \nu}^{(\kappa)} \mathcal{T}_{n(r)-\rho(\sigma), \mu}^{(\rho)} \frac{I_{\rho(\sigma)-\bar{n}(r)}\left(\frac{\bar{z}_{\rho}}{\bar{z}_{\kappa}}, 0\right)}{\rho(\sigma)}\right. \\
= & \mathcal{G}^{-n(r), \mu ; n(r) \nu}(\sigma)\left(1-\delta_{\bar{n}(r), 0}\right) \\
& \times\left(\mathcal{T}_{n(r) \nu}^{(\rho)} \mathcal{T}_{-n(r), \mu}^{(\kappa)} I_{\bar{n}(r)}\left(\frac{z_{\rho}}{z_{\kappa}}, 0\right)+\mathcal{T}_{n(r) \nu}^{(\kappa)} \mathcal{T}_{-n(r), \mu}^{(\rho)} I_{\bar{n}(r)}\left(\frac{z_{\rho}}{\bar{z}_{\kappa}}, 0\right)\right) \\
= & \mathcal{G}^{n(r), \mu ;-n(r), \nu}(\sigma)\left(1-\delta_{\bar{n}(r), 0}\right) \\
& \times\left(\mathcal{T}_{n(r) \mu}^{(\rho)} \mathcal{T}_{-n(r), \nu}^{(\kappa)} I_{\frac{\bar{n}(r)}{\rho(\sigma)}}\left(\frac{z_{\rho}}{z_{\kappa}}, 0\right)+\mathcal{T}_{n(r) \mu}^{(\kappa)} \mathcal{T}_{-n(r), \nu}^{(\rho)} I_{\bar{n}(r)}\left(\frac{\bar{z}_{\rho}}{\bar{z}_{\kappa}}, 0\right)\right) \\
= & F(\rho, \kappa)+\Delta \\
\Delta \equiv & \mathcal{G}^{n(r) \mu ;-n(r), \nu}(\sigma)\left(1-\delta_{\bar{n}(r), 0}\right)\left(\mathcal{T}_{n(r) \mu}^{(\rho)} \mathcal{T}_{-n(r), \nu}^{(\kappa)}+\mathcal{T}_{n(r) \mu}^{(\kappa)} \mathcal{T}_{-n(r), \nu}^{(\rho)}\right) I_{\frac{\bar{n}(r)}{\rho(\sigma)}}(\infty, 0)
\end{aligned}
$$

Here we have used the identity 2.97a) and the periodicity $n(r) \rightarrow n(r) \pm \rho(\sigma)$ of $\mathcal{G}$ and $\mathcal{T}$ to obtain (C.2a $)$ and the variable change $n^{\prime} \equiv \rho-n$ to obtain ( $(\mathrm{C} .2 \mathrm{E})$ are obtained with the symmetry of $\mathcal{G}$ and a $\mu \leftrightarrow \nu$ relabelling.

The same steps on the first term of $\Delta$ are used to show that $\Delta=0$

$$
\begin{aligned}
& \mathcal{G}^{n(r) \mu ;-n(r), \nu}(\sigma)\left(1-\delta_{\bar{n}(r), 0}\right) \mathcal{T}_{-n(r), \nu}^{(\kappa)} \mathcal{T}_{n(r) \mu}^{(\rho)} I_{\frac{\bar{n}(r)}{\rho(\sigma)}}(\infty, 0) \\
= & \mathcal{G}^{n(r)-\rho(\sigma), \mu ; \rho(\sigma)-n(r), \nu}(\sigma)\left(1-\delta_{\bar{n}(r), 0}\right) \mathcal{T}_{\rho(\sigma)-n(r), \nu}^{(\kappa)} \mathcal{T}_{n(r)-\rho(\sigma), \mu}^{(\rho)} \frac{\rho(\sigma)-\bar{n}(r)}{\rho(\sigma)}(0, \infty) \\
= & \mathcal{G}^{-n(r), \mu ; n(r) \nu}(\sigma)\left(1-\delta_{\bar{n}(r), 0}\right) \mathcal{T}_{n(r) \nu}^{(\kappa)} \mathcal{T}_{-n(r), \mu}^{(\rho)} I_{\frac{\bar{n}(r)}{\rho(\sigma)}}(0, \infty) \\
= & -\mathcal{G}^{n(r) \mu ;-n(r), \nu}(\sigma)\left(1-\delta_{\bar{n}(r), 0}\right) \mathcal{T}_{n(r) \mu}^{(\kappa)} \mathcal{T}_{-n(r), \nu}^{(\rho)} \frac{I_{\frac{\bar{n}(r)}{\rho(\sigma)}}}{(\infty), 0)}
\end{aligned}
$$

which establishes the symmetry of $F$. 


\section{Relation to the Dynkin automorphisms}

Our first exercise in this appendix is to show explicitly that the standard action $\tau$ of the Dynkin diagram automorphism of $\mathfrak{s u}(3)$

$$
\begin{gathered}
\tau\left(\alpha_{1}\right)=\alpha_{2}, \quad \tau\left(\alpha_{2}\right)=\alpha_{1}, \quad \tau(\alpha \cdot H)=\alpha \cdot \tau(H)=\tau(\alpha) \cdot H \\
\tau\left(E_{ \pm \alpha_{1}}\right)=E_{ \pm \alpha_{2}}, \quad \tau\left(E_{ \pm \alpha_{2}}\right)=E_{ \pm \alpha_{1}}, \quad \tau\left(E_{ \pm\left(\alpha_{1}+\alpha_{2}\right)}\right)=-E_{ \pm\left(\alpha_{1}+\alpha_{2}\right)} \\
\alpha_{1}=(1,0), \quad \alpha_{2}=\frac{1}{2}(-1, \sqrt{3})
\end{gathered}
$$

is inner-automorphically equivalent $\tau \simeq \omega$ to the action of the outer automorphism $\omega$ in the Cartesian basis of the text. As seen in (D.1d), we take $\alpha^{2}=1$ for our discussion of $\mathfrak{s u}(3)$.

We present this equivalence in a set of relations

$$
\begin{aligned}
& (-) \quad J_{1}=\tilde{J}_{5}=\frac{1}{i \sqrt{2}}\left(E_{\alpha_{1}+\alpha_{2}}-E_{-\left(\alpha_{1}+\alpha_{2}\right)}\right) \\
& (+) \quad J_{2}=\frac{1}{2}\left(\tilde{J}_{3}+\sqrt{3} \tilde{J}_{8}\right)=\left(\alpha_{1}+\alpha_{2}\right) \cdot H \\
& (-) \quad J_{3}=\tilde{J}_{4}=\frac{1}{\sqrt{2}}\left(E_{\alpha_{1}+\alpha_{2}}+E_{-\left(\alpha_{1}+\alpha_{2}\right)}\right) \\
& (-) \quad J_{4}=\frac{1}{\sqrt{2}}\left(\tilde{J}_{7}-\tilde{J}_{2}\right)=\frac{i}{2}\left(E_{\alpha_{1}}-E_{\alpha_{2}}-\left(E_{-\alpha_{1}}-E_{-\alpha_{2}}\right)\right) \\
& (+) \quad J_{5}=\frac{1}{\sqrt{2}}\left(\tilde{J}_{1}+\tilde{J}_{6}\right)=\frac{1}{2}\left(E_{\alpha_{1}}+E_{\alpha_{2}}+E_{-\alpha_{1}}+E_{-\alpha_{2}}\right) \\
& (-) \quad J_{6}=\frac{1}{\sqrt{2}}\left(\tilde{J}_{1}-\tilde{J}_{6}\right)=\frac{1}{2}\left(E_{\alpha_{1}}-E_{\alpha_{2}}+E_{-\alpha_{1}}-E_{-\alpha_{2}}\right) \\
& (+) \quad J_{7}=\frac{1}{\sqrt{2}}\left(\tilde{J}_{2}+\tilde{J}_{7}\right)=\frac{1}{2 i}\left(E_{\alpha_{1}}+E_{\alpha_{2}}-\left(E_{-\alpha_{1}}+E_{-\alpha_{2}}\right)\right) \\
& (-) \quad J_{8}=\frac{1}{2}\left(\sqrt{3} \tilde{J}_{3}-\tilde{J}_{8}\right)=\frac{1}{\sqrt{3}}\left(\alpha_{1}-\alpha_{2}\right) \cdot H
\end{aligned}
$$

whose derivation and meaning we discuss below.

In $(\overline{\mathrm{D} .2})$ the generators $J^{\dagger}=J$ are the Cartesian generators of the text, while the generators $\tilde{J}^{\dagger}=\tilde{J}$ are another set of Cartesian generators related to the Cartan-Weyl generators as follows:

$$
\begin{gathered}
E_{ \pm \alpha_{1}}=\frac{1}{\sqrt{2}}\left(\tilde{J}_{1} \pm i \tilde{J}_{2}\right), \quad E_{ \pm \alpha_{2}}=\frac{1}{\sqrt{2}}\left(\tilde{J}_{6} \pm i \tilde{J}_{7}\right), \quad E_{ \pm\left(\alpha_{1}+\alpha_{2}\right)}=\frac{1}{\sqrt{2}}\left(\tilde{J}_{4} \pm i \tilde{J}_{5}\right) \\
, \quad H=\left(\tilde{J}_{3}, \tilde{J}_{8}\right), \quad E_{\alpha}^{\dagger}=E_{-\alpha}, \quad H^{\dagger}=H .
\end{gathered}
$$

The $\tilde{J} \leftrightarrow(E, H)$ relations in (D.2) give the $\tilde{J}$ form of the hermitian eigenstates of the Cartan-Weyl generators under $\tau$. The signs $( \pm)$ shown on the far left of (D.2) are the eigenvalues of all the combinations under $\tau \simeq \omega$.

The relation between the $J$ 's and the $\tilde{J}$ 's is in fact an inner automorphism of $\mathfrak{s u}(3)$. To see this explicitly, consider the unitary matrix

$$
U=\frac{e^{-i \pi / 6}}{\sqrt{2}}\left(\begin{array}{ccc}
i & 1 & 0 \\
0 & 0 & \sqrt{2} \\
i & -1 & 0
\end{array}\right), \quad U^{\dagger} U=\mathbb{1}, \quad \operatorname{det} U=1
$$


Then we find by explicit computation that

$$
\begin{gathered}
U^{\dagger}\left\{\frac{\lambda_{1}+\lambda_{6}}{\sqrt{2}}, \frac{\lambda_{2}+\lambda_{7}}{\sqrt{2}}, \frac{\lambda_{3}+\sqrt{3} \lambda_{8}}{2}, \lambda_{4}, \frac{\sqrt{3} \lambda_{3}-\lambda_{8}}{2}, \frac{\lambda_{1}-\lambda_{6}}{\sqrt{2}}, \frac{\lambda_{7}-\lambda_{2}}{\sqrt{2}}, \lambda_{5}\right\} U \\
=\left\{\lambda_{5}, \lambda_{7}, \lambda_{2}, \lambda_{3}, \lambda_{8}, \lambda_{6}, \lambda_{4}, \lambda_{1}\right\}
\end{gathered}
$$

where $\lambda_{a}, a=1 \ldots 8$ are the $3 \times 3$ Gell-Mann matrices. This result shows that the relation in (D.2) between the $J$ 's and $\tilde{J}$ 's is an automorphism, and moreover this automorphism is an inner automorphism because $U$ in (D.4) is an element of the Lie group $S U(3)$.

To see this for all representations of $\mathfrak{s u}(3)$, note that the matrix $U$ can be written in the form $U=\exp (i \beta \cdot T)$ for $T$ the fundamental of $\mathfrak{s u}(3)$. Then the relations (D.5) imply the same forms as operator relations with $U \rightarrow \tilde{U}=\exp (i \beta \cdot J)$ and $\lambda_{a} \rightarrow J_{a}$ for any set of Cartesian generators, including $J$ or $\tilde{J}$.

Two further comments are in order. We mention first that the matrix $U$ in (D.4) is nothing but a particular matrix of eigenvectors of $\lambda_{4}$. Repeating the computation with the most general matrix of eigenvectors of $\lambda_{4}$ gives a more general form of (D.5) which shows the inner-automorphic equivalence of all the irregularly embedded $\mathfrak{s o}(3)$ 's in $\mathfrak{s u}(3)$. Second, the $J \leftrightarrow(E, H)$ relations in (D.2) can also be used to check the equivalence of our Cartesian form of $A_{2}^{(2)}$ in (3.21) with the conventional form of $A_{2}^{(2)}$ obtained directly from the action of the Dynkin automorphism. One need only hat the operators in the $J \leftrightarrow(E, H)$ relations

$$
\begin{gathered}
J \rightarrow \hat{J}, \quad E_{ \pm\left(\alpha_{1}+\alpha_{2}\right)} \rightarrow \hat{E}_{ \pm\left(\alpha_{1}+\alpha_{2}\right)}, \quad H \rightarrow \hat{H} \\
E_{ \pm \alpha_{1}}+E_{ \pm \alpha_{2}} \rightarrow E_{ \pm \alpha_{1}+E_{ \pm \alpha_{2}}}, \quad E_{ \pm \alpha_{1}}-E_{ \pm \alpha_{2}} \rightarrow E_{ \pm \alpha_{1}-E_{ \pm \alpha_{2}}}
\end{gathered}
$$

being careful as shown to maintain the eigenstates of $\tau$.

For $\mathfrak{s u}(2 n+1)$ it is well known [19] that there is a realization of the Dynkin automorphism with invariant subalgebra $h=\mathfrak{s o}(2 n+1)$. This is easily checked starting from the trivial phase $\tau\left(E_{\alpha_{i}}\right)=E_{\tau\left(\alpha_{i}\right)}$ for all simple roots.

For $\mathfrak{s u}(2 n)$ however it is known [19] that there are two inner-automorphically equivalent realizations of the Dynkin automorphism, resulting in a choice of invariant subalgebra $h=\mathfrak{s o}(2 n)$ or $h=\mathfrak{c}_{n}$. This is not difficult to check explicitly, starting from the positive roots

$$
\alpha_{i j}=e_{i}-e_{j}, \quad e_{i} \cdot e_{j}=\delta_{i j}, \quad \alpha_{i j}^{2}=2, \quad 1 \leq i<j \leq 2 n
$$

(with simple roots $\alpha_{i, i+1}$ ) and their corresponding generators $E_{i j} \equiv E_{\alpha_{i j}}$ :

$$
\left[E_{i k}, E_{k j}\right]=E_{i j}, \quad \tau\left(\alpha_{i, i+1}\right)=\alpha_{2 n-i, 2 n-i+1}, \quad \tau\left(\alpha_{i j}\right)=\alpha_{2 n-j+1,2 n-i+1} .
$$

The $n$ invariant positive roots are $\alpha_{i, 2 n-i+1}, i=1 \ldots n$, including the invariant simple root $\alpha_{n, n+1}$.

By comparison with $\left[\tau\left(E_{i k}\right), \tau\left(E_{k j}\right)\right]=\tau\left(E_{i j}\right)$ we find that the general automorphism has the form

$$
\tau\left(E_{i j}\right)=\xi_{i j} E_{2 n-j+1,2 n-i+1}, \quad \xi_{i j}=(-)^{j-i-1}\left(\prod_{l=1}^{j-1} \xi_{l, l+1}\right)
$$


where $\xi_{i, i+1}$ is the phase of the simple root generator $E_{i, i+1}$. Moreover, the form (D.9) is unique given the "initial condition" $\left\{\xi_{i, i+1}\right\}$. The two realizations of the Dynkin automorphism are then found by distinguishing the sign of the invariant simple root

$$
\begin{array}{rll}
\text { I. } \forall & \xi_{i, i+1}=1 & \rightarrow h=\mathfrak{c}_{n} \\
\text { II. } \forall \quad \xi_{i, i+1}=1 \text { except } \xi_{n, n+1}=-1 & \rightarrow h=\mathfrak{s o}(2 n) .
\end{array}
$$

As far as counting is concerned, the difference between the two cases is the sign of the $n$ invariant positive roots

$$
\tau\left(E_{i, 2 n-i+1}\right)=\xi_{i, 2 n-i+1} E_{i, 2 n-i+1}, \quad \xi_{i, 2 n-i+1}=\left\{\begin{array}{ll}
+1 & \text { for I } \\
-1 & \text { for II }
\end{array}, \quad i=1 \ldots n .\right.
$$

The same is found for the $n$ invariant negative roots, and the relation

$$
\operatorname{dim}\left(\mathfrak{c}_{n}\right)=\operatorname{dim}(\mathfrak{s o}(2 n))+2 n=n(2 n+1)
$$

reflects this difference between cases I and II. Finally, it is easily checked with (D.10) and the fundamental representation of $\mathfrak{s u}(2 n)$ that the composition of automorphisms I and II is an inner automorphism of $\mathfrak{s u}(2 n)$.

\section{E Flat connections in the charge conjugation orbifolds}

To check explicitly that the twisted $\mathrm{KZ}$ connection (3.410) is flat, we will need only the symmetricspace form $\left(f_{I J K}=0\right)$ of the algebra (3.26b), (3.260), (3.26d) of the twisted representation matrices. In further detail, we will use only the following list of simple identities

$$
\begin{gathered}
\frac{1}{2 z_{\mu \nu} \sqrt{z_{\mu} z_{\nu}}} \pm \frac{1}{z_{\mu \nu}^{2}}\left(\frac{z_{\nu}}{z_{\mu}}\right)^{ \pm \frac{1}{2}}= \pm \frac{z_{\mu}+z_{\nu}}{2 z_{\mu \nu}^{2} \sqrt{z_{\mu} z_{\nu}}} \\
{\left[\mathcal{T}_{0 A}, \sum_{I} \mathcal{T}_{1 I} \mathcal{T}_{1 I}\right]=0} \\
\frac{1}{z_{\mu \rho} z_{\nu \rho}}=\frac{1}{z_{\mu \rho} z_{\nu \mu}}+\frac{1}{z_{\mu \nu} z_{\nu \rho}} \\
\frac{1}{z_{\mu \rho} z_{\nu \rho}} \frac{z_{\rho}}{\sqrt{z_{\mu} z_{\nu}}}-\frac{1}{z_{\mu \rho} z_{\nu \mu}}\left(\frac{z_{\mu}}{z_{\nu}}\right)^{\frac{1}{2}}-\frac{1}{z_{\mu \nu} z_{\nu \rho}}\left(\frac{z_{\nu}}{z_{\mu}}\right)^{\frac{1}{2}}=0 \\
\sum_{I J A} i f_{I J A}\left(\mathcal{T}_{1 I}^{(\nu)} \mathcal{T}_{1 J}^{(\nu)} \mathcal{T}_{0 A}^{(\mu)}-(\mu \leftrightarrow \nu)\right)=\frac{1}{2} \sum_{I J A} i f_{I J A}\left(\left[\mathcal{T}_{1 I}^{(\nu)}, \mathcal{T}_{1 J}^{(\nu)}\right] \mathcal{T}_{0 A}^{(\mu)}-(\mu \leftrightarrow \nu)\right) \\
=\frac{1}{2} \sum_{I J A B} i f_{I J A} i f_{I J B}\left(\mathcal{T}_{0 B}^{(\nu)} \mathcal{T}_{0 A}^{(\mu)}-(\mu \leftrightarrow \nu)\right)=0 \\
\sum_{I J A} i f_{A I J}\left(\mathcal{T}_{0 A}^{(\mu)} \mathcal{T}_{1 J}^{(\nu)} \mathcal{T}_{1 I}^{(\mu)}+\mathcal{T}_{1 J}^{(\mu)} \mathcal{T}_{1 I}^{(\nu)} \mathcal{T}_{0 A}^{(\mu)}-(\mu \leftrightarrow \nu)\right)=\sum_{I J A} i f_{A I J}\left(\left[\mathcal{T}_{0 A}^{(\mu)}, \mathcal{T}_{1 J}^{(\nu)} \mathcal{T}_{1 I}^{(\mu)}\right]-(\mu \leftrightarrow \nu)\right)
\end{gathered}
$$




$$
=\sum_{I J A K} i f_{A I J} i f_{A I K}\left(\mathcal{T}_{1 J}^{(\nu)} \mathcal{T}_{1 K}^{(\mu)}-(\mu \leftrightarrow \nu)\right)=0
$$

where $\mu, \nu$ and $\rho$ are distinct. The identity (E.1d) follows from (E.1d) and many identities of this form were needed [10] in the explicit check of flatness of the twisted KZ connections of the WZW cyclic permutation orbifolds. The identities (E.1b), (E.1G) and (E.1f) are simple consequences of the symmetric-space form of the algebra of twisted representation matrices.

Then by differentiation and (E.1a) we find that

$$
\partial_{\mu} \hat{W}_{\nu}-\partial_{\nu} \hat{W}_{\mu}=\frac{2}{2 k+Q_{g}}\left[\sum_{A} \mathcal{T}_{0 A}^{(\mu)} \mathcal{T}_{0 A}^{(\nu)}+\frac{z_{\mu}+z_{\nu}}{2 \sqrt{z_{\mu} z_{\nu}}} \sum_{I} \mathcal{T}_{1 I}^{(\mu)} \mathcal{T}_{1 I}^{(\nu)}\right]-(\mu \leftrightarrow \nu)=0
$$

In what follows, we sketch the more difficult check that

$$
\left[\hat{W}_{\mu}, \hat{W}_{\nu}\right]=0 \text {. }
$$

For this computation, we introduce the symbolic notation

$$
\hat{W}_{\mu}=h_{\mu}+(g / h)_{\mu}+e_{\mu}
$$

where $h$ denotes the untwisted subalgebra terms and so on. Then we find using (E.1a), (E.1b) and (E.1d) that

$$
\left[e_{\mu}, e_{\nu}\right]=\left[e_{\mu}, h_{\nu}\right]=\left[h_{\mu}, e_{\nu}\right]=\left[h_{\mu}, h_{\nu}\right]=0 .
$$

The last relation in this list is not surprising because $h$ is an untwisted subalgebra.

After some algebra, the rest of the terms can be divided into summed terms $\sum_{\rho \neq \mu, \nu}$ and unsummed terms which involve only $z_{\mu}$ and $z_{\nu}$. For example one finds that the commutator

$$
\begin{aligned}
{\left[(g / h)_{\mu},(g / h)_{\nu}\right]=} & \sum_{I J A} i f_{I J A} \sum_{\rho \neq \mu, \nu}\left\{\frac{1}{z_{\mu \rho} z_{\nu \mu}}\left(\frac{z_{\rho}}{z_{\nu}}\right)^{\frac{1}{2}} \mathcal{T}_{1 I}^{(\rho)} \mathcal{T}_{0 A}^{(\mu)} \mathcal{T}_{1 J}^{(\nu)}\right. \\
& \left.+\frac{1}{z_{\mu \rho} z_{\nu \rho}} \frac{z_{\rho}}{\sqrt{z_{\mu} z_{\nu}}} \mathcal{T}_{0 A}^{(\rho)} \mathcal{T}_{1 J}^{(\nu)} \mathcal{T}_{1 I}^{(\mu)}+\frac{1}{z_{\mu \nu} z_{\nu \rho}}\left(\frac{z_{\rho}}{z_{\mu}}\right)^{\frac{1}{2}} \mathcal{T}_{1 J}^{(\rho)} \mathcal{T}_{0 A}^{(\nu)} \mathcal{T}_{1 I}^{(\mu)}\right\}
\end{aligned}
$$

has no unsummed terms, which have cancelled according to $(\mathrm{E} .1 \mathrm{~d})$. Using $(\mathrm{E} .1 \mathrm{~d})$ and $(\mathrm{E} .1 \mathrm{~d})$ the summed terms (E.6) are found to cancel against the summed terms from $\left[h_{\mu},(g / h)_{\nu}\right]+\left[(g / h)_{\mu}, h_{\nu}\right]$. This leaves the unsummed terms from this sum of commutators plus those from $\left[e_{\mu},(g / h)_{\nu}\right]+$ $\left[(g / h)_{\mu}, e_{\nu}\right]$. Using (E.1f $)$, we find that all these terms sum to zero

$$
-\frac{1}{z_{\mu \nu}} \frac{z_{\mu}+z_{\nu}}{2 \sqrt{z_{\mu} z_{\nu}}} \sum_{I J A} i f_{I J A}\left(\mathcal{T}_{0 A}^{(\mu)} \mathcal{T}_{1 J}^{(\nu)} \mathcal{T}_{1 I}^{(\mu)}+\mathcal{T}_{1 J}^{(\mu)} \mathcal{T}_{1 I}^{(\nu)} \mathcal{T}_{0 A}^{(\mu)}\right)=0
$$

which completes the check of flatness.

One may also check the consistency relation between the twisted KZ equation (3.41b) and the global Ward identity (3.41d)

$$
\left[\hat{W}_{\mu}(\mathcal{T}, z), \sum_{\mu=1}^{N} \mathcal{T}_{0 A}^{(\mu)}\right]=0, \quad \forall A \in \mathfrak{s o}(n)
$$

but this is left as an exercise for the reader. 


\section{References}

[1] L. Borisov, M. B. Halpern, and C. Schweigert, "Systematic approach to cyclic orbifolds," Int. J. Mod. Phys. A13 (1998) 125, hep-th/9701061.

[2] J. Evslin, M. B. Halpern, and J. E. Wang. "General Virasoro construction on orbifold affine algebra," Int. J. Mod. Phys. A14 (1999) 4985, hep-th/9904105.

[3] J. de Boer, J. Evslin, M. B. Halpern, and J. E. Wang, "New duality transformations in orbifold theory," Int. J. Mod. Phys. A15 (2000) 1297, hep-th/9908187.

[4] M. B. Halpern and,J. E. Wang, "More about all current-algebraic orbifolds," Int. J. Mod. Phys. A16 (2001) 97, hep-th/0005187.

[5] K. Bardakci and M. B. Halpern, "New dual quark models," Phys. Rev. D3 (1971) 2493.

[6] M. B. Halpern, "The two faces of a dual pion-quark model," Phys. Rev. D4 (1971) 2398.

[7] M. B. Halpern and E. Kiritsis, "General Virasoro construction on affine g," Mod. Phys. Lett. A4 (1989) 1373.

[8] M. B. Halpern, E. B. Kiritsis, and N. A. Obers, "The Lie $h$ invariant conformal field theories and the Lie $h$ invariant graphs," Int. J. Mod. Phys. A7 (1992) s339, hep-th/9110001.

[9] M. B. Halpern, E. Kiritsis, N. A. Obers, and K. Clubok, "Irrational conformal field theory," Physics Reports 265 (1996) 1-138, hep-th/9501144.

[10] J. de Boer, M. B. Halpern, and N. A. Obers, "The operator algebra and twisted KZ equations of WZW orbifolds," JHEP 10 (2001) 011, hep-th/0105305.

[11] J. Evslin, M. B. Halpern, and J. E. Wang, "Cyclic coset orbifolds," Int. J. Mod. Phys. A15 (2000) 3829-3860, hep-th/9912084.

[12] M. B. Halpern and N. A. Obers, "New semiclassical nonabelian vertex operators for chiral and nonchiral WZW theory," Int. J. Mod. Phys. A12 (1997) 4317, hep-th/9610081.

[13] E. Corrigan and D. B. Fairlie, "Off-shell states in dual resonance theory," Nucl. Phys. B91 (1975) 527.

[14] J. Lepowsky and R. L. Wilson, "Construction of the affine Lie algebra $A_{1}^{(1)}$," Commun. Math. Phys. 62 (1978) 43-53.

[15] J. Lepowsky and M. Primc, "Structure of the standard modules for the affine Lie algebra $A_{1}^{(1)}$," Contemp. Math. 46 (1985) 1.

[16] C. Dong and J. Lepowsky, "The algebraic structure of relative twisted vertex operators," J. Pure Appl. Algebra 110 (1996) 259-295.

[17] S. Fubini and G. Veneziano, "Duality in operator formalism," Nuovo Cim. 67A (1970) 29.

[18] M. B. Halpern and C. B. Thorn, "Two faces of a dual pion-quark model. 2. Fermions and other things," Phys. Rev. D4 (1971) 3084-3088.

[19] V. Kac, Infinite Dimensional Lie Algebras, Third Edition, Cambridge University Press, 1990.

[20] L. Birke, J. Fuchs, and C. Schweigert, "Svmmetrv breaking boundary conditions and WZW orbifolds," $A d v$. Theor. Math. Phys. 3 (1999) 671-726, hep-th/9905038.

[21] R. Wendt, "Weyl's character formula for non-connected Lie groups and orbifold theory for twisted affine Lie algebras," J. Funct. Anal. 180 (2001) 31-65.

[22] V. B. Petkova and J. B. Zuber, "Boundary conditions in charge conjugate $s l(N)$ WZW theories," hep-th/0201239.

[23] R. Dashen and Y. Frishman, "Four fermion interactions and scale invariance," Phys. Rev. D11 (1975) 2781.

[24] V. G. Knizhnik and A. B. Zamolodchikov, "Current algebra and Wess-Zumino model in two dimensions," Nucl. Phys. B247 (1984) 83-103. 